\title{
Wireless Physiological Monitoring System for Psychiatric Patients
}

by

\begin{abstract}
AJ Rademeyer
Thesis presented at the University of Stellenbosch in partial fulfilment of the requirements for the degree of
\end{abstract}

Master of Science in Mechatronic Engineering Department of Mechanical and Mechatronic Engineering Stellenbosch University

Project Supervisors:

Prof. Cornie Scheffer

Dr. Mike Blanckenberg

December 2008 


\section{DECLARATION}

By submitting this dissertation electronically, I declare that the entirety of the work contained therein is my own, original work, that I am the owner of the copyright thereof (unless to the extent explicitly stated otherwise) and that I have not previously in its entirety or in part submitted it for obtaining any qualification.

Date: Desember, 2008

Copyright (C) 2008 Stellenbosch University

All rights reserved 


\title{
ABSTRACT
}

\section{Wireless Physiological Monitoring System for Psychiatric Patients}

\author{
AJ Rademeyer \\ Department of Mechanical and Mechatronic Engineering \\ Stellenbosch University \\ Private Bag X1, 7602 Matieland, South Africa \\ Thesis: MScEng (Mechatronic)
}

December 2008

\begin{abstract}
This thesis is concerned with the development and testing of a non-invasive device that is unassailable, and can be placed on an aggressive psychiatric patient to monitor the vital signs of this patient. Two devices, a glove measuring oxygen saturation and another on the dorsal part (back) of the patient measuring heart rate via electrocardiography (ECG), skin temperature and respiratory rate were designed and implemented. The data is transmitted using wireless technology. Both devices connect to one central monitoring station using two separate Bluetooth connections ensuring a total wireless setup. All the hardware and software to measure these variables have been designed and implemented. A Matlab graphical user interface (GUI) was developed for signal processing and monitoring of the vital signs of the psychiatric patient. Detection algorithms were implemented to detect ECG arrhythmias such as premature ventricular contraction and atrial fibrillation. The prototype was manufactured and tested in a laboratory setting on five volunteers. Satisfactory test results were obtained and the primary objectives of the thesis were fulfilled.
\end{abstract}




\title{
UITTREKSEL
}

\section{Draadlose Fisiologiese Observasiesisteem vir Psigiatriese Pasiënte}

\author{
AJ Rademeyer \\ Departement van Meganiese en Megatroniese Ingenieurswese \\ Stellenbosch Universiteit \\ Privaatsak X1, 7602 Matieland, Suid-Afrika \\ Tesis: MScIng (Megatronies)
}

Desember 2008

\begin{abstract}
Hierdie tesis handel oor die ontwerp en toets van 'n ekstern gekoppelde toestel wat op 'n aggressiewe psigiatriese pasiënt geplaas kan word en wat die belangrikste lewens voorwaardes (sg. "vitale tekens") van die pasiënt monitor met draadlose elektroniese tegnologie. Daar bestaan twee stelsels: die een is 'n handskoen wat die suurstofsaturasie meet, en die ander word gemonteer op die rug en meet die harttempo d.m.v. elektrokardiografie (EKG), vel temperatuur en asemhalingstempo. Albei hierdie stelsels word gekonnekteer met 'n sentrale observasie-eenheid met twee aparte "Bluetooth" koppelings wat verseker dat 'n totale draadlose stelsel gerealiseer word. Al die sensors, drade en elektronika is geïnstalleer in 'n veiligheidsomhulsel. 'n Matlab grafiese verbruikersintervlak (GVI) word gebruik vir seinverwerking en observasie van belangrikste lewens voorwaardes van psigiatriese pasiënte. Algoritmes is geïmplementeer om EKG aritmieë soos 'n ekstra sistool en atriale fibrillasie te herken. Die prototipe is vervaardig en getoets in die laboratorium op vyf vrywilliges. Aanvaarbare toetsresultate is gemeet en die primêre doelwitte van die projek is behaal.
\end{abstract}




\section{DEDICATION}

To Liezl 


\section{ACKNOWLEDGEMENTS}

The author would like to thank the following people for their contribution towards this project:

- To my parents, Danie and Carla, thank you for granting me this opportunity.

- Dr. DJE Rademeyer for financial and motivational support.

- Prof. Cornie Scheffer, my supervisor, for his continuous guidance throughout the project.

- Dr. Mike Blankenberg, my co-supervisor, for his continuous guidance throughout the project.

- Dr. Gunter Winkler for his inputs towards the realization of the project and for organizing a meeting at Stikland Psychiatric Hospital.

- Ferdie Zietsman, Cobus Zietsman and Anton van den Berg of the Mechanical \& Mechatronic Departments Workshop for the manufacturing of the project parts.

- JP Conradie for the help with Matlab programming and computer aided design (CAD) design.

- PJ Christiane for help with CAD design.

- AN Smuts for inputs related to the selection of components and design considerations.

- K Becker for his assistance in the development of the sound transducer system.

- Liezl Conradie for continuous motivational support.

- Prof. Piet J. Jonas for spelling and grammar inspection. 


\section{CONTENTS}

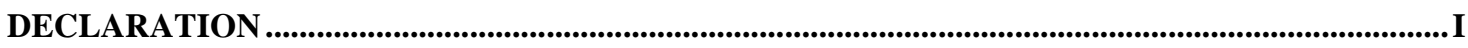

ABSTRACT …..................................................................................................................................... II

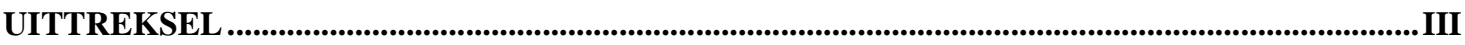

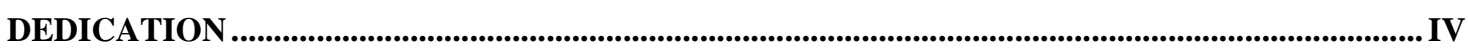

ACKNOWLEDGEMENTS .............................................................................................................................

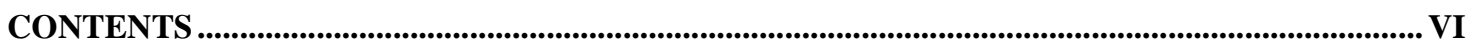

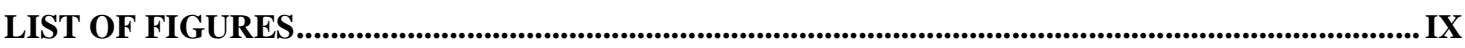

LIST OF TABLES........................................................................................................................................ XI

GLOSSARY \& NOMENCLATURE ........................................................................................XII

CHAPTER 1 ............................................................................................................................................. 1

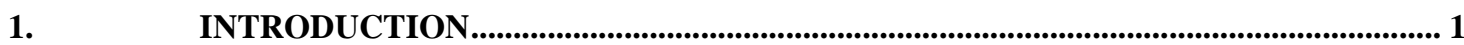

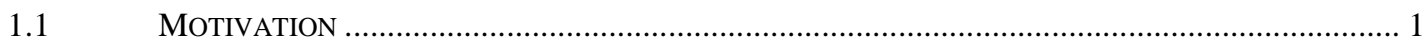

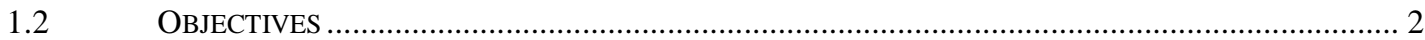

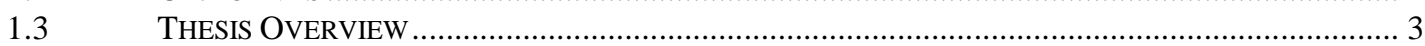

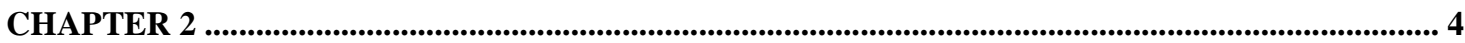

2. LITERATURE REVIEW AND RATIONALE ...................................................................... 4

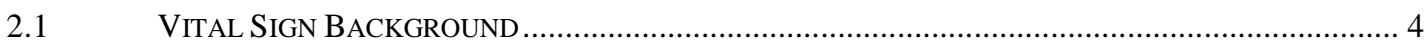

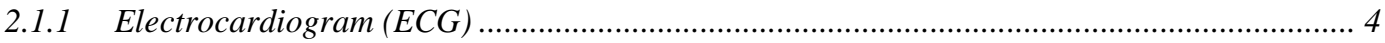

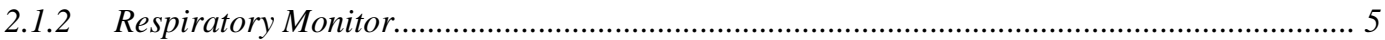

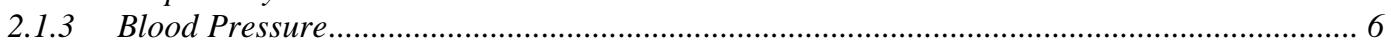

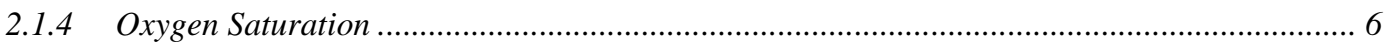

2.2 PORTABLE (AMBULATORY) HEALTH MONITORING DEVICES ................................................ 7

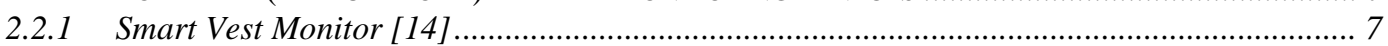

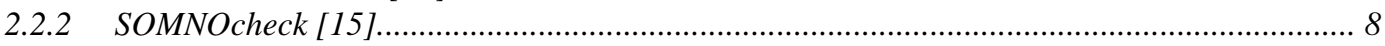

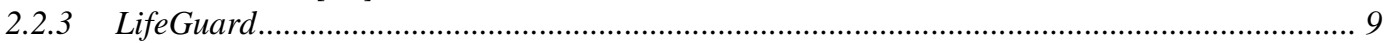

2.2.4 Programmable Wireless Electrode System for Medical Monitoring [17] ................................ 9

2.2.5 Vital Sign Remote Monitoring Device [18]...........................................................................10

2.2.6 Wireless Medical Diagnosis and Monitoring Equipment [19] ...........................................10

2.2.7 Method and Apparatus for Monitoring Physiological Parameters of a Living Subject [20]..11

2.2.8 Device System and Method for Monitoring Vital Signs [21] ..............................................12

2.2.9 Wearable Health Care System for Vital Sign Monitoring [22]............................................13

2.2.10 A WBAN (Wireless Body Area Networks) System for Ambulatory Monitoring of Physical Activity and Health Status [23]................................................................................... 13

2.2.11 AMON: A Wearable Multiparameter Medical Monitoring and Alert System [24]................14

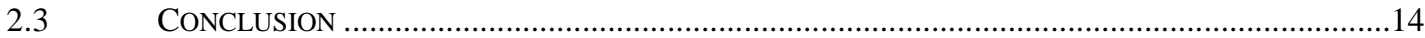

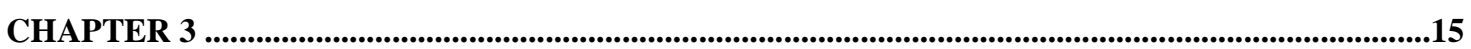

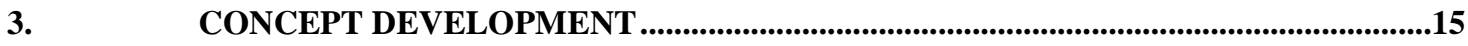

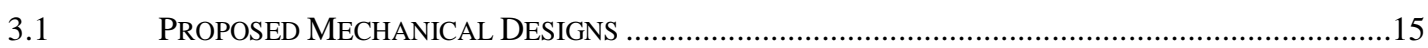

SAFETY CUFF CONCEPT

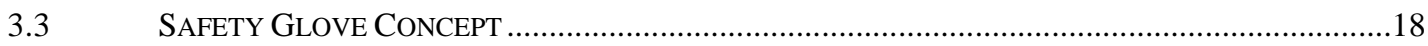

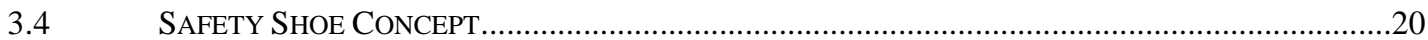

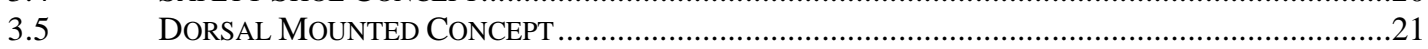

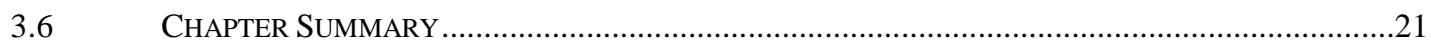

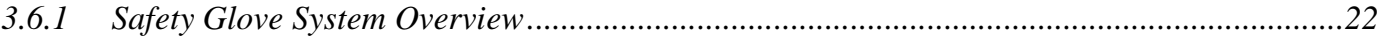


3.6.2 Dorsal Mounted System Overview ...........................................................................22

3.6.3 Central Monitoring Station Overview.......................................................................22

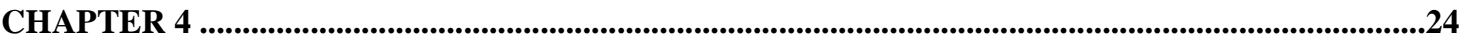

4.

DEVELOPMENT OF THE DORSAL MOUNTED DEVICE............................................24

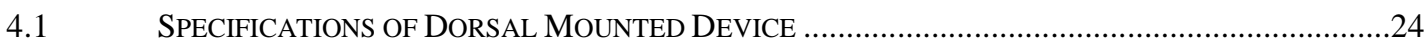

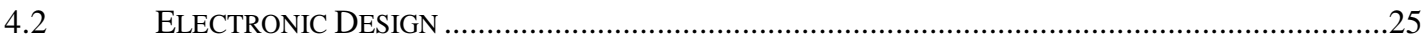

4.2.1 Selection of Main Electronic Components ....................................................................26

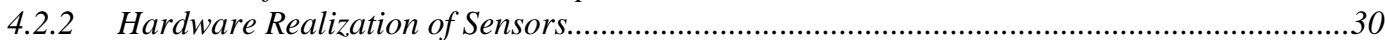

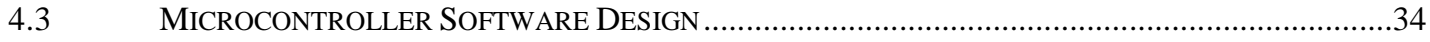

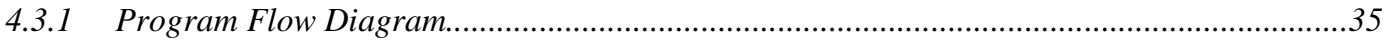

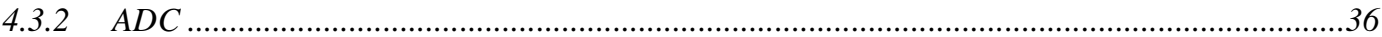

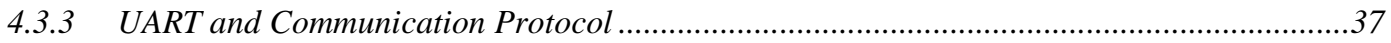

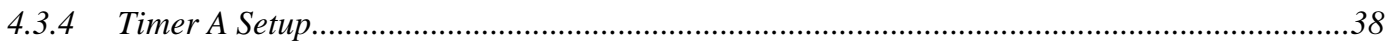

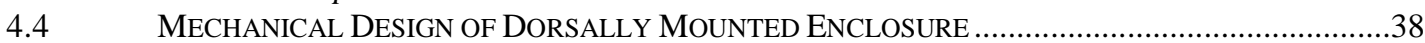

4.4.1 Design Considerations for Each Sensor ..............................................................................38

4.4.2 CAD Prototype Design of Electronic Protection Enclosure …............................................43

4.4.3 Manufacturing of the Prototype Protection Enclosure .........................................................44

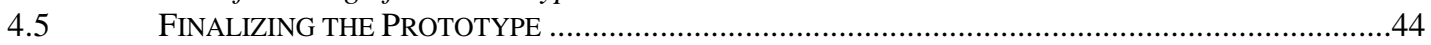

4.5.1 Combining Electronics and Mechanical Prototype ...........................................................44

4.5.2 Proposed Fitting of Prototype to the Patient .....................................................................45

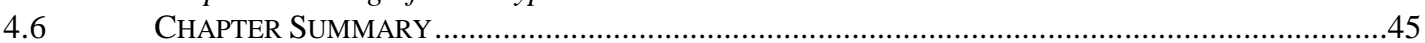

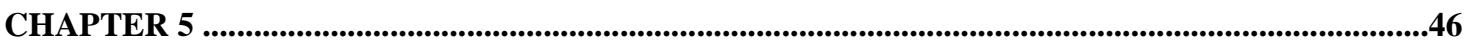

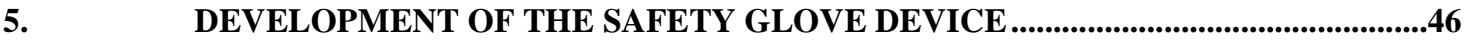

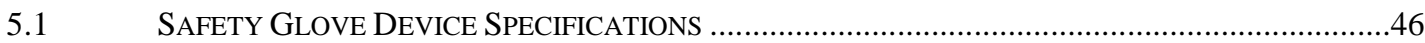

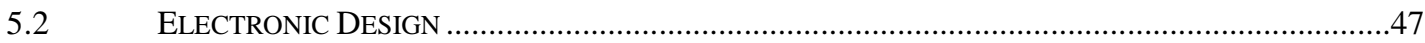

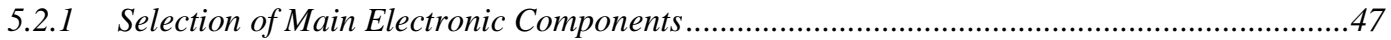

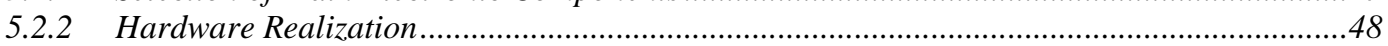

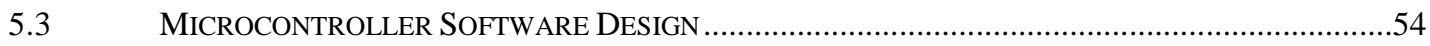

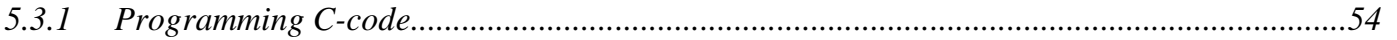

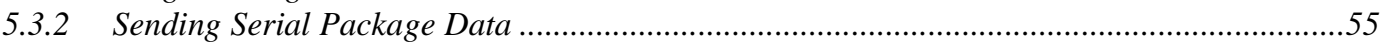

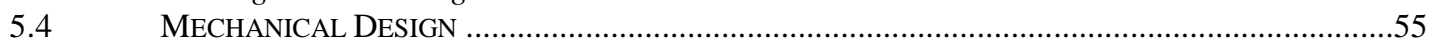

5.4.1 CAD Design and Selection of Electronic Protection Enclosure .........................................55

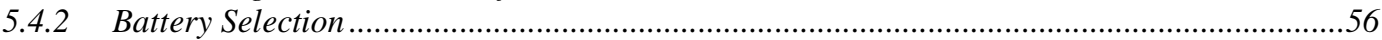

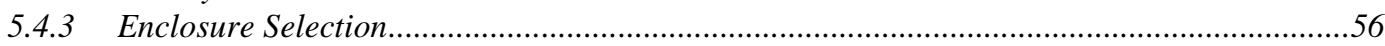

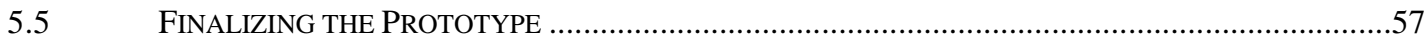

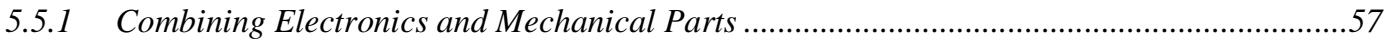

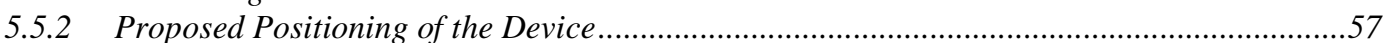

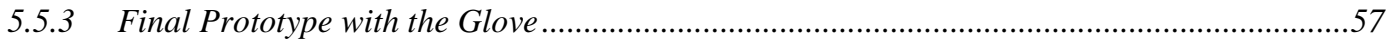

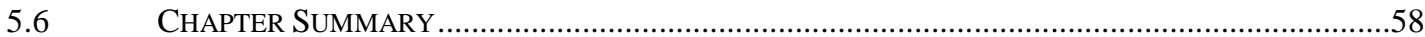

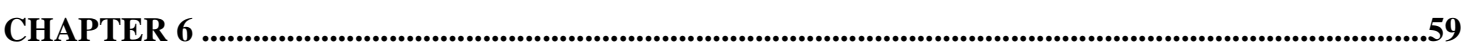

6.

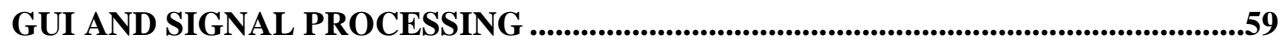

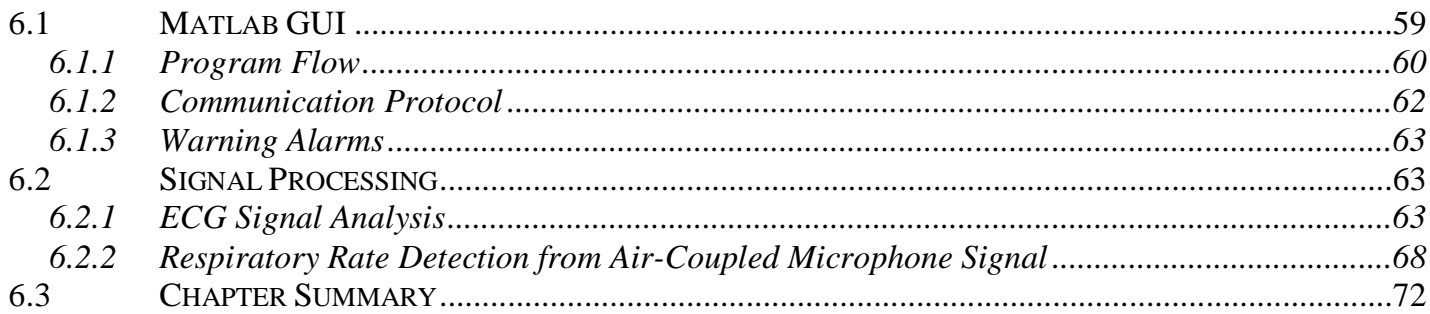


CHAPTER 7 ....................................................................................................................................................................73

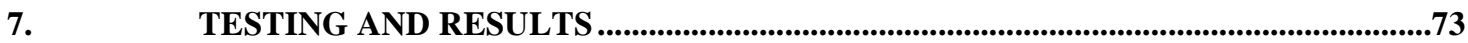

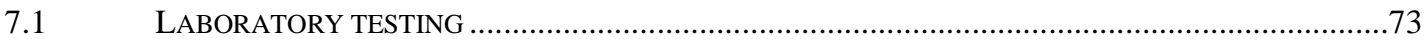

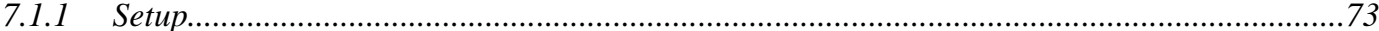

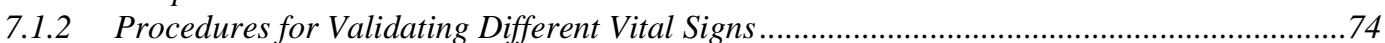

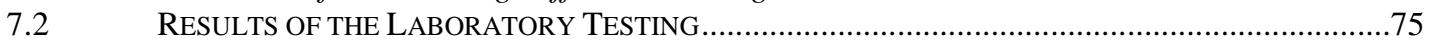

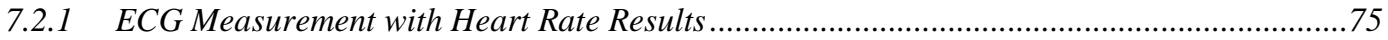

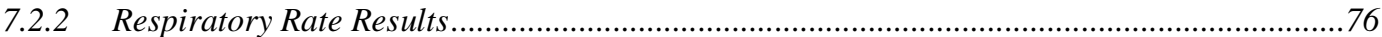

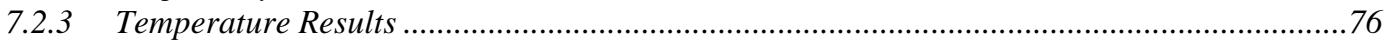

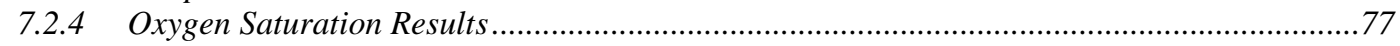

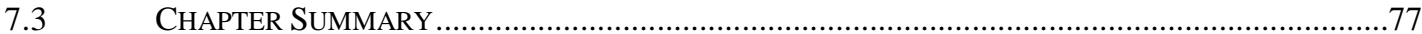

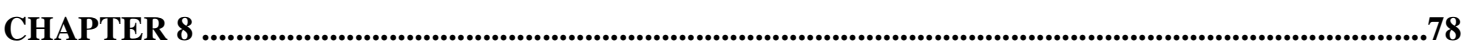

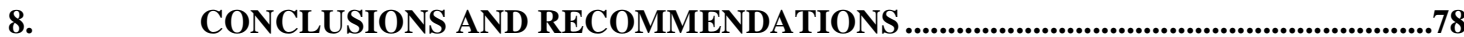

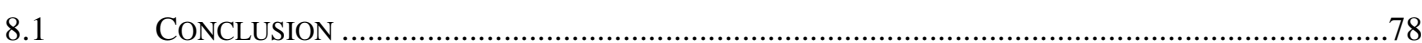

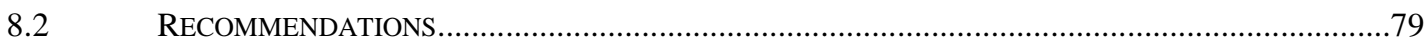

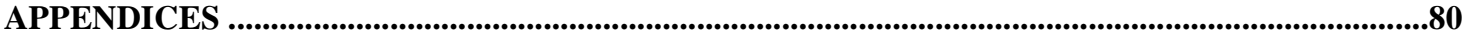

APPENDIX A1 - CONTINUOUS NON-INVASIVE BlOOD PRESSURE MONITOR ……........................................80

APPENDIX A2 - COMMERCIAL AND PATENTED MONITORING DEVICES ................................................89

APPENDIX A3 - WIRELESS COMMERCIAL PUlSE OXIMETER PRODUCTS …........................................91

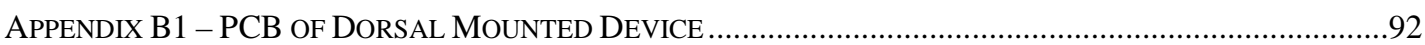

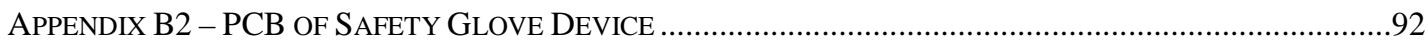

APPENDIX C1 - CAD DESIGN OF DORSAL MOUNTED DEVICE ..........................................................93

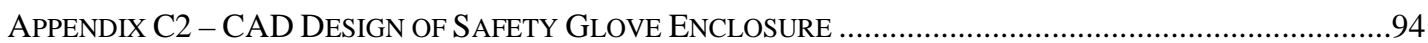

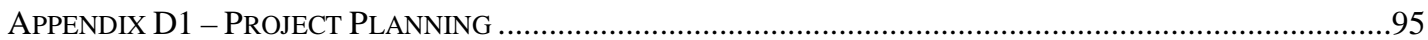

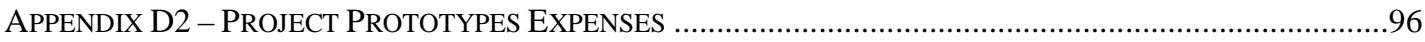

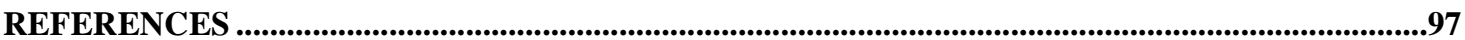




\section{LIST OF FIGURES}

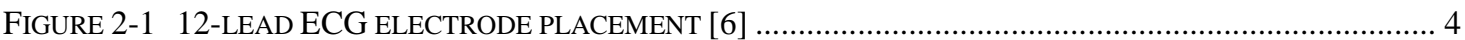

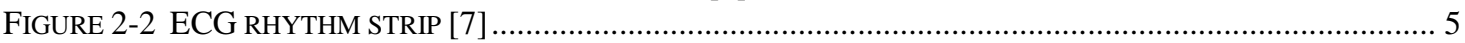

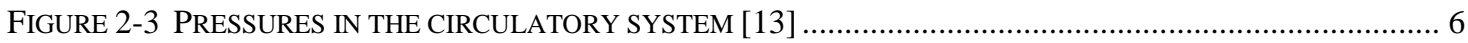

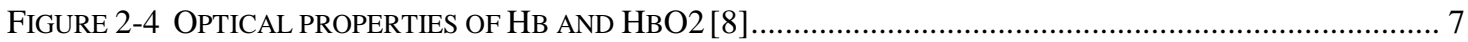

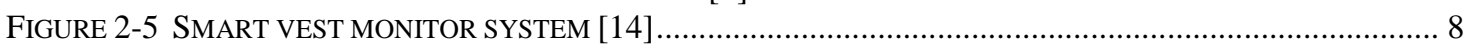

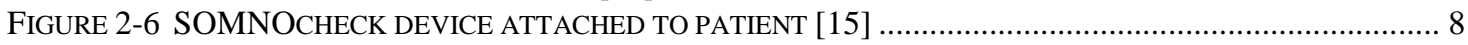

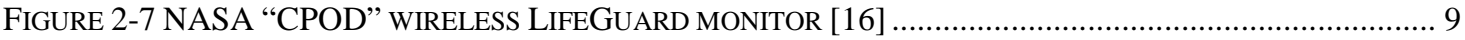

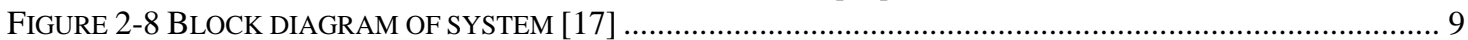

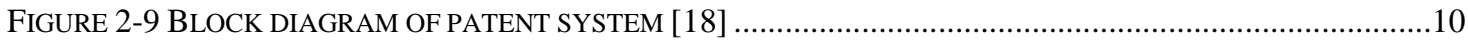

FIGURE 2-10 ARRANGEMENT OF ELECTRODES PLACEMENT ON PATIENT [19] ..........................................11

FIGURE 2-11 PHYSIOLOGICAL PARAMETERS MONITORING SYSTEM [20] ...............................................11

FIGURE 2-12 BLOCK DIAGRAM OF PHYSIOLOGICAL PARAMETERS MONITORING SYSTEM [20] ......................12

FIGURE 2-13 BLOCK DIAGRAM AND ACCELEROMETER OUTPUT WITH HAND AT REST AND IN MOTION [21] ....13

FIGURE 2-14 WRIST WORN MEDICAL DEVICE WITH GSM/UMTS LINK TO TELEMEDICINE CENTRE [24].........14

FIGURE 3-1 REFLECTANCE PROBE PLACEMENT OVER RADIAL ARTERY ON WRIST .......................................17

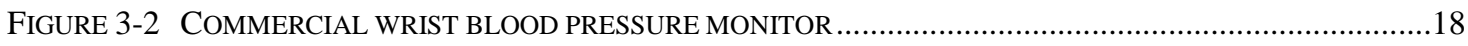

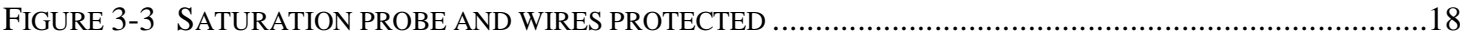

FIGURE 3-4 A SCHEMATIC DIAGRAM FOR ESTIMATING BLOOD PRESSURE [26] ..........................................19

FIGURE 3-5 EIP CIRCUIT WITH MODULATED 50 KHZ SINUSOIDAL SIGNAL ............................................20

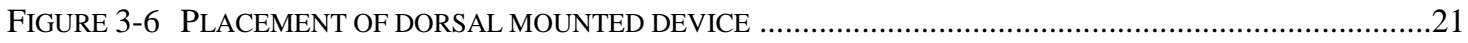

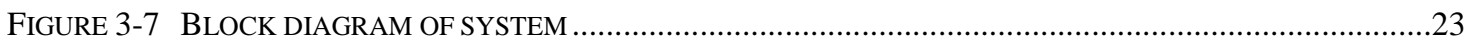

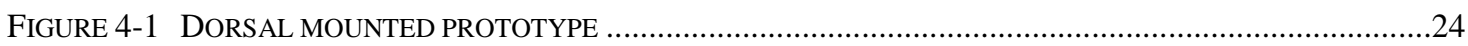

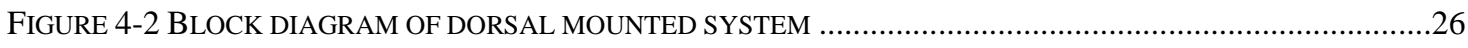

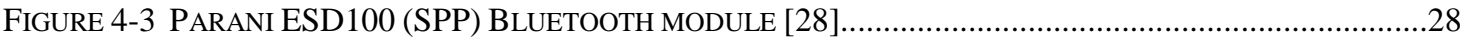

FIGURE 4-4 Jig BOARD TO SIMULATE SERIAL CABLE USING TWO BLUETOOTH MODULES [29] .........................29

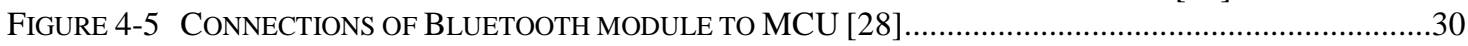

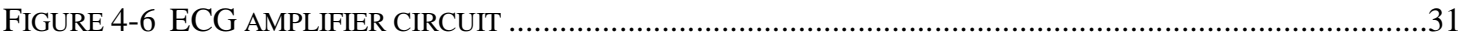

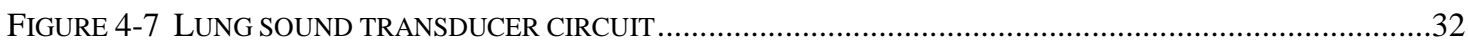

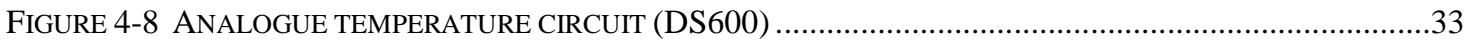

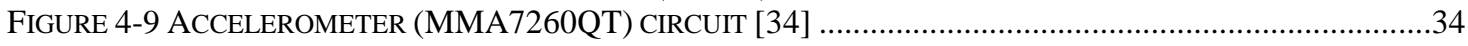

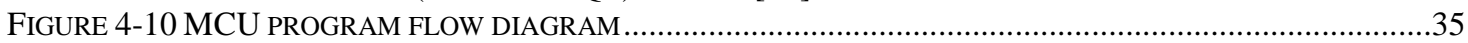

FIGURE 4-11 REPEATED SEQUENCE OF CHANNELS ADC SAMPLING [35] ....................................................36

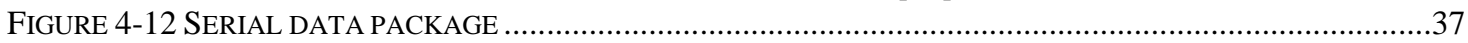

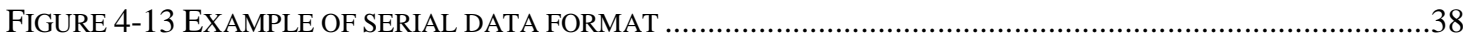

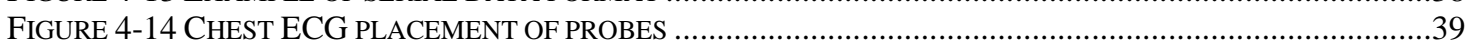

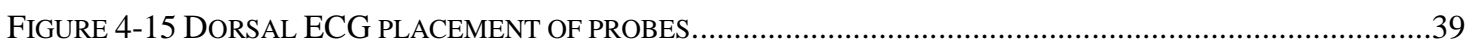

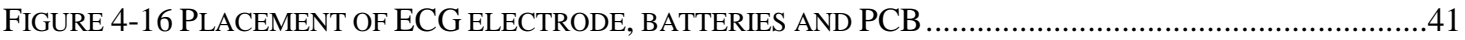

FIGURE 4-17 ECG CLIP AND PROBE FITTED TO SPRING LOADED SYSTEM ..................................................42

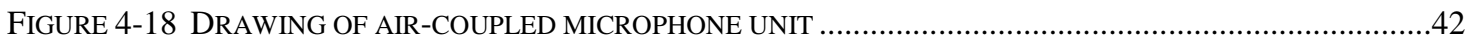

FIGURE 4-19 CAD MODEL INCLUDING BATTERIES, PCB AND BLUETOOTH MODULE..................................44

FIGURE 4-20 FITTING ELECTRONICS INSIDE DORSAL MOUNTED DEVICE ..................................................45

FIGURE 4-21 ADHESIVE PLASTER ATTACHMENT OF DEVICE ON BACK OF A VOLUNTEER ...............................45

FIGURE 5-1 FINAL PROTOTYPE WITHOUT THE PROTECTIVE GLOVE ….......................................................46

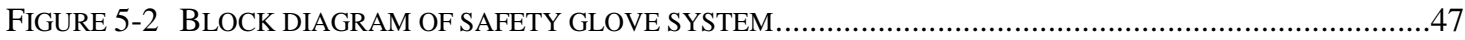

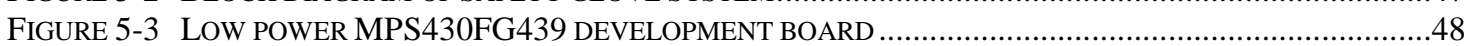

FIGURE 5-4 BLOCK DIAGRAM OF PULSE OXIMETER SYSTEM [44] .........................................................49

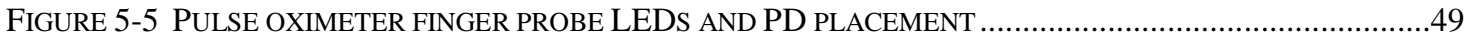

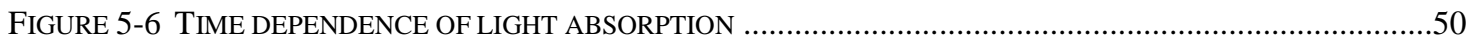

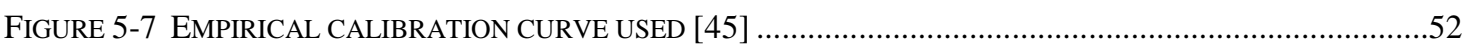

FIGURE 5-8 H-BRIDGE CIRCUIT FOR DIGITAL SWITCHING OF LEDS [44] .............................................52

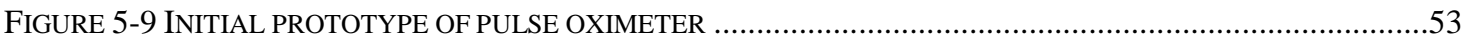

FIGURE 5-10 DISPLAY MEASURED PULSATILE RED AND INFRARED PPG SIGNALS ........................................53

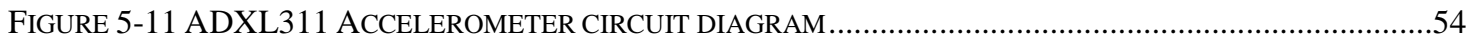




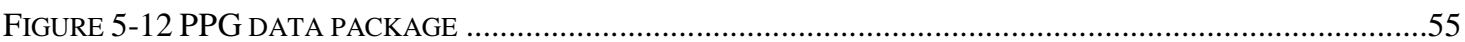

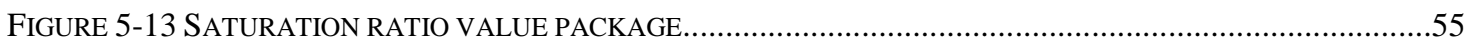

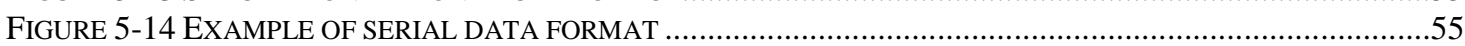

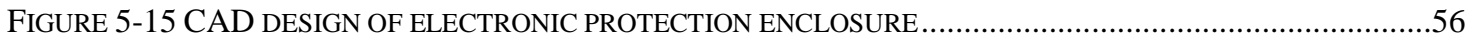

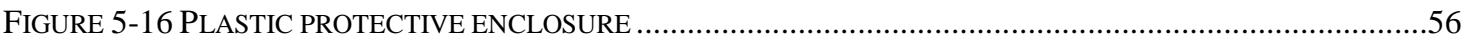

FIGURE 5-17 PROTECTION ENCLOSURE WITH PCB INSTALLED AND CLOSED .............................................57

FIGURE 5-18 VELCRO RUBBER PROTECTION AND VELCRO STRAP …...................................................57

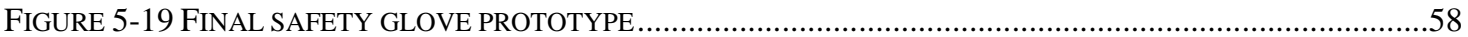

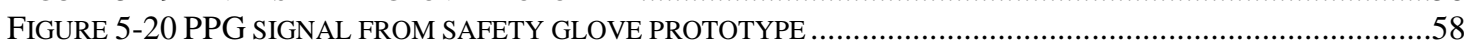

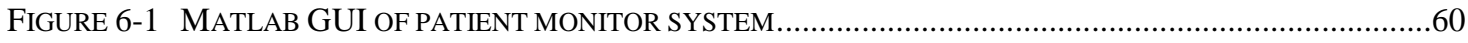

FIGURE 6-2 MATLAB SOFTWARE PROGRAM FLOW DIAGRAM ...............................................................61

FIGURE 6-3 DORSAL MOUNTED DEVICE DATA PACKAGE RECEIVED FROM MCU ........................................62

FIGURE 6-4 SAFETY GLOVE DEVICE DATA PACKAGE RECEIVED FROM MCU ............................................62

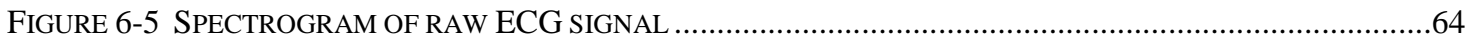

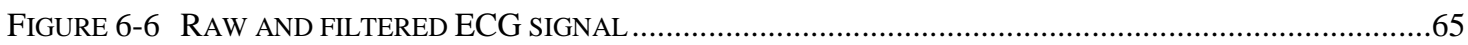

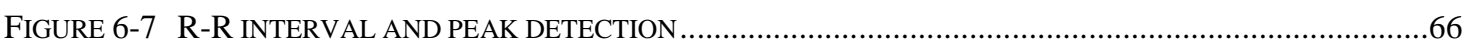

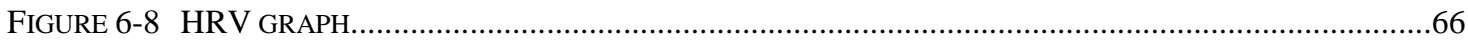

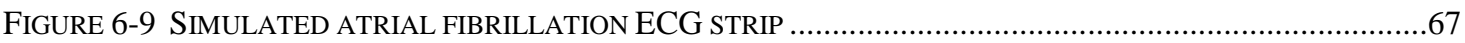

FIGURE 6-10 HRV GRAPH WITH THREE ATRIAL FIBRILLATION POINTS ....................................................67

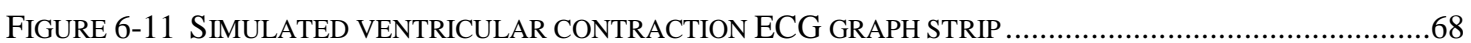

FIGURE 6-12 HRV GRAPH WITH THREE PVC POINTS DETECTED ........................................................68

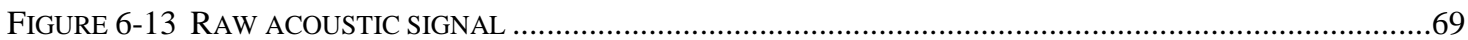

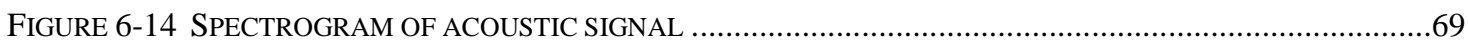

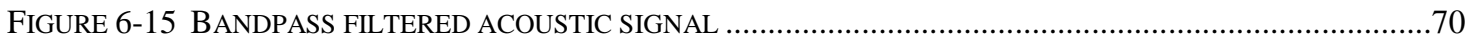

FIGURE 6-16 RAW, BANDPASS, ENVELOPE FILTERED AND PEAK DETECTION SOUND DATA ..........................71

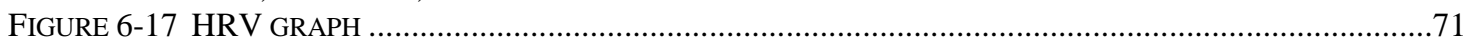

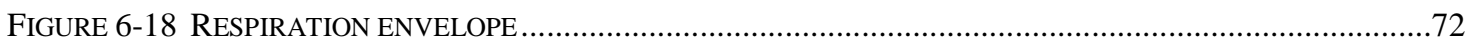

FIGURE 7-1 PROTOTYPE ATTACHED TO A VOLUNTEER USING A RUBBER BAND .........................................73

FIGURE A-1 PORTAPRES PORTABLE CONTINUOUS BLOOD PRESSURE MONITOR [ [57] ....................................80

FIGURE A-2 VOLUME CLAP METHODOLOGY AND MECHANISM [58] ..................................................81

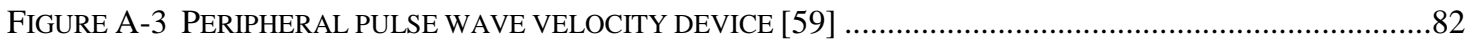

FIGURE A-4 COMPARISON OF PWV DATA AND BP DATA AT TWO DIFFERENT HEIGHTS [59] .......................83

FIGURE A-5 SCHEMATIC BLOCK DIAGRAM OF THE PROTOTYPE SYSTEM [60] .............................................8

FIGURE A-6 UPSTREAM, DIGITAL ARTERIAL SEGMENT AND DOWNSTREAM MODEL [26] .............................85

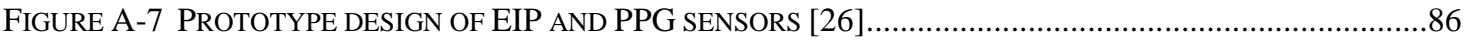

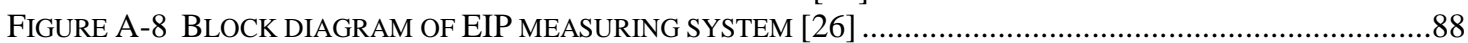

FIGURE A-9 ALIVE WIRELESS BLUETOOTH ENABLED PULSE OXIMETER [73] ............................................91

FIGURE A-10 NONIN 4100 BLUETOOTH ENABLED PULSE OXIMETER [74] ................................................91

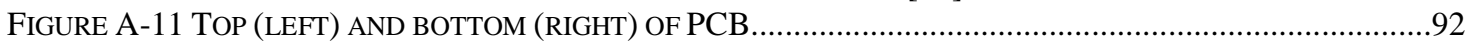

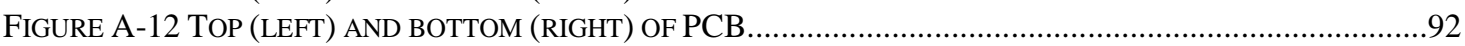

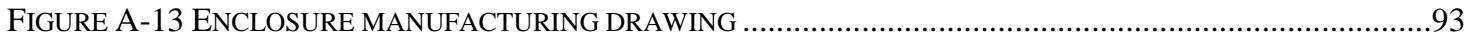

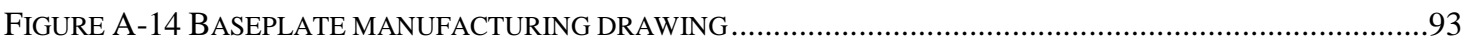

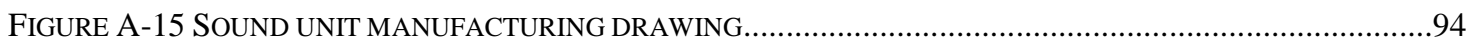

FIGURE A-16 ELECTRONIC PROTECTION ENCLOSURE MANUFACTURING DRAWING .....................................99

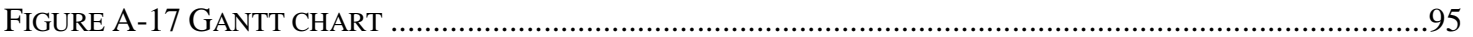




\section{LIST OF TABLES}

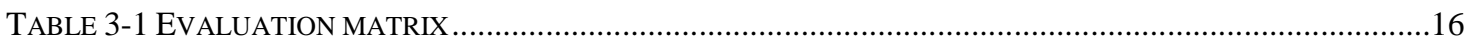

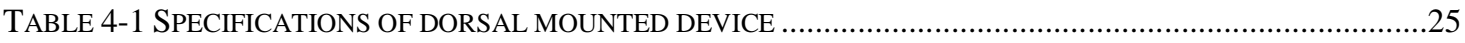

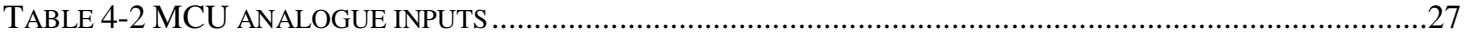

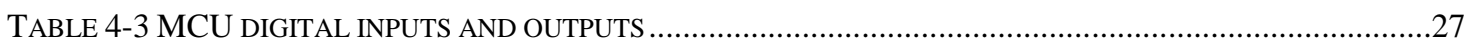

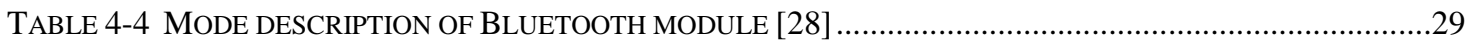

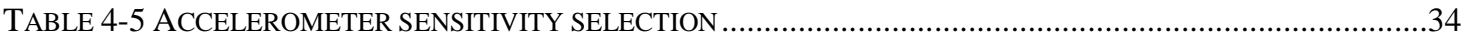

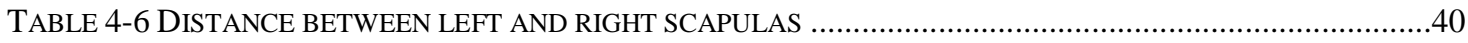

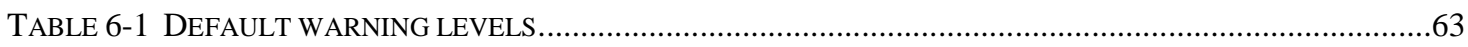

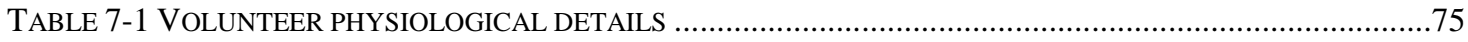

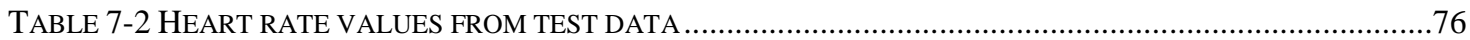

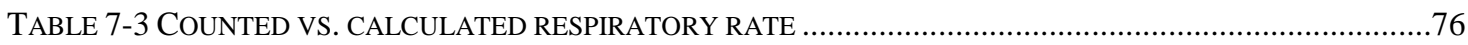

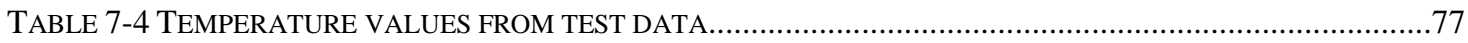

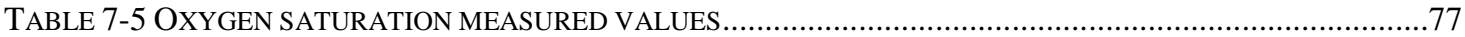

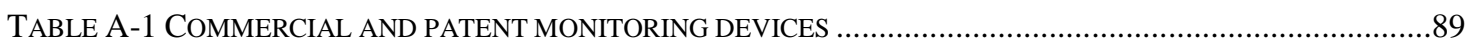

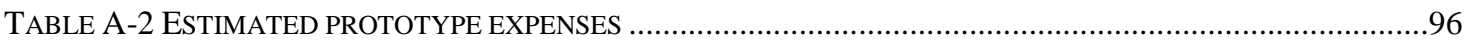




\section{GLOSSARY \& NOMENCLATURE}

\section{GLOSSARY}

Acute psychosis

Apnea

Arrhythmias

Atrial fibrillation

Bluetooth

Diastolic blood pressure

Diazepam

Dorsal

Hypotension

Hypothermia

Lorazepam

Matlab

Plethysmograph

Premature ventricular contraction
- Any short, severe mental disorder in which contact with reality is lost or highly distorted.

- Transient cessation of respiration.

- An abnormal rate of muscle contractions in the heart.

- Fibrillation of the muscles of the atria of the heart.

- Wireless communication protocol.

- Lower blood pressure value, shows the pressure exerted on the walls of the arteries when your heart is in the relaxation phase (diastole), measured in $\mathrm{mmHg}$.

- A tranquilizer (trade name Valium) used to relieve anxiety and relax muscles.

-Belonging to or on or near the back or upper surface of an animal or organ or part.

- Abnormally low blood pressure.

- Subnormal body temperature.

- Tranquilizer (trade name Ativan) used to treat anxiety and tension and insomnia.

- Software program to implement GUI and digital signal processing.

- A measuring instrument for measuring changes in volume of a part or organ or whole body (usually resulting from fluctuations in the amount of blood it contains).

- Irregularity of cardiac rhythm; recurrent occurrences can be a precursor of ventricular fibrillation. 
Psychotic

Pulse oximeter

Sedation

Seclusion

Spectrogram

Systolic blood pressure

\section{VARIABLES}

$f$
$f_{s}$
$n(t)$
$P$
$T_{R R}$
$T H_{s}$
$T_{x}$
$T R_{s}$
$T R_{x}$

\section{ABBREVIATIONS}

\section{$\mathrm{AC}$}

ACC

Acc_average

ACLK

ADC

AF

ARR

BM1

BM2

BPM

BP

BPS

CAD

CMRR

CMS
- Characteristic of or suffering from psychosis.

- A measuring instrument that measures the oxygen in arterial blood.

-The administration of a sedative agent or drug.

-The quality of being secluded from the presence or view of others.

- A photographic record of a spectrum.

- Higher blood pressure value, the pressure in your arteries when your heart is forcing blood through them, measured in $\mathrm{mmHg}$.

- frequency

- sampling frequency

- noise signal

- power of a signal

- R-R interval duration

- one divided by the sampling frequency

- R-R interval

- one divided by the sampling frequency

- peak to peak for RR detection

- alternating current

- accelerometer

- average value of three accelerometer values

- auxiliary clock

- analogue to digital converter

- atrial fibrillation

- average respiratory rate

- bluetooth module 1

- bluetooth module 2

- beats per minute

- blood pressure

- bits per second

- computer aided design

- common mode rejection ratio

- component monitoring system 


$\begin{array}{ll}\text { DAC } & \text { - digital to analogue converter } \\ \text { DC } & \text { - direct current } \\ \text { DCO } & \text { - digital controller oscillator } \\ \text { DSP } & \text { - digital signal processing } \\ \text { ECG } & \text { - electrocardiograph } \\ \text { EEG } & \text { - electroencephalogram } \\ \text { EIP } & \text { - electrical impedance plethysmograph } \\ \text { EOS } & \text { - end of sequence } \\ \text { EPC } & \text { - electro pneumatic converter } \\ \text { GPS } & \text { - global positioning system } \\ \text { GSR } & \text { - galvanic skin response } \\ \text { GUI } & \text { - graphical user interface } \\ \text { Hb } & \text { - a hemoprotein composed of globin and heme } \\ \text { HbO } & \text { - microcirculatory hemoglobin oxygen saturation } \\ \text { HR } & \text { - heart rate } \\ \text { HRV } & \text { - heart rate variability } \\ \text { IR } & \text { - infrared light } \\ \text { LCD } & \text { - liquid crystal display } \\ \text { LED } & \text { - light-emitting diode } \\ \text { MCU } & \text { - microcontroller } \\ \text { OEM } & \text { - original equipment manufacturer } \\ \text { PC } & \text { - personal computer } \\ \text { PCB } & \text { - printed circuit board } \\ \text { PD } & \text { - photo diode } \\ \text { PID } & \text { - proportional integral differential } \\ \text { PPG } & \text { - photo plethysmography } \\ \text { PTT } & \text { - pulse transit time } \\ \text { PVC } & \text { - premature ventricular contraction } \\ \text { PWV } & \text { - pulse wave velocity } \\ \text { R } & \text { - red light } \\ \text { RF } & \text { - radio frequency } \\ \text { RMS } & \text { - root mean square } \\ \text { RR } & \text { - respiratory rate } \\ \text { SOO }_{2} & \text { - oxygen saturation } \\ \text { SB } & \text { - start byte } \\ \text { SNR } & \text { - signal to noise ratio } \\ \text { Sound_L } & \text { - sound low byte } \\ \text { Sound_H } & \text { - sound high byte } \\ \text { SPP } & \text { - serial port profile } \\ \text { TEMP } & \text { - temperature } \\ \text { UART } & \text { - universal asynchronous receiver/transmitter } \\ & \end{array}$


INTRODUCTION

\section{CHAPTER 1}

\section{INTRODUCTION}

Psychotic patients in psychiatric hospitals that are sedated or secluded are sometimes at risk of death or injury if they are not continuously monitored. In 1998 a number of visible incidents that can be linked to the restraining and sedation of patients led to the deaths of adults and children in the psychiatric setting [1]. Therefore Hartford Courant [1] commissioned a study that revealed that between 50 and 150 deaths occur each year in psychiatric environments in the USA are due to the use of seclusion or restraints [2]. There is hence the need for a psychotic patient monitoring device that monitors and sends the vital signs of a psychotic patient wirelessly from the device worn by the patient to a central monitoring station during sedation or seclusion situations. This device will assist clinicians to monitor patients continuously and more efficiently without any physical contact with the patient.

Previously a patient in seclusion could only be monitored by the clinician using a video camera, or by visiting the patient. If the patient is sleeping, the clinician has no knowledge of whether the patient's vital signs are stable without personal clinical observation. With the proposed device one clinician can monitor several patients from central monitoring stations and if any of the vital sign data transmitted and calculated on a central monitoring station is outside of the selected margins, an alarm will be activated to ensure that the clinician on duty can attend to that patient as soon as possible. The type of vital signs of healthy humans are the same as that of psychotic patients and therefore the vital signs that will be monitored with this device are oxygen saturation, pulse rate, electrocardiography (ECG), respiratory rate and skin temperature. Other measurements such as movement of the patient are used to determine if the patient is moving uncontrollably, which will activate a "motion" alarm.

\subsection{Motivation}

During psychiatric emergencies such as acute psychosis, behaviour is sometimes troubled, with either vicious or physically aggressive behaviour. In these circumstances there is a need to ensure the safety of the patient, the society, and health workers. At the same time, the human rights of the patient to privacy, dignity and autonomy need to be respected. This situation often creates immense difficulties for nurses and doctors. In order to manage difficult activities, clinicians always attempt to defuse the behaviour by techniques such as "talking down". If this is not successful, clinicians use either sedation (with oral or injectable medication) or 


\section{INTRODUCTION}

seclusion (restraint in a locked room). Bodily restraint, using devices such as restraining belts, is used in some countries, but is not currently used in South Africa. The monitoring of the vital signs of a psychotic patient is an enormous problem for the clinicians working at a psychiatric hospital. When the patient is actively walking around there is little danger of a medical emergency. The problem occurs when the patient feels tired and is lying down because of the sedatives such as Lorazepam or Diazepam. The patient will have the tendency to lie down, which causes difficulties for the clinician, since the clinician cannot establish the patient's vital signs with video monitoring alone while the patient is sleeping. When the patient is sleeping he/she can develop apnea (suspension of external breathing) or hypotension (abnormally low blood pressure), which can be fatal if not treated at an early stage.

During sedation or seclusion, the physical condition of the patient has to be monitored (implying variables such as blood pressure, pulse rate, respiration, temperature etc.) every 30 minutes as required by the Mental Health Care Act, 2002, Under the Act, Regulation 7578 of 2003; Section 39 (2) [3]. Monitoring of the physical state is often inadequate due to a combination of staff shortage and fears of staff for their own security. Deaths of psychiatric patients during seclusion have occurred in psychiatric hospitals [2]. The use of the small device that monitors the vital signs of the patient is necessary to protect the patient from hypotension or other medical emergencies.

The proposed device that will be attached to the patient will assist the clinician and the patient since the physical contact time with the patient and the clinician will be minimised. A device that constantly monitors the patients' physical condition will contribute to the safety of patients during psychiatric emergencies and reduce a considerable workload of medical staff.

\subsection{Objectives}

The primary objective of the project is to develop a compact psychotic patient vital sign monitoring device. This includes the continuous monitoring of different vital signs such as ECG, heart rate, respiratory rate, temperature, oxygen saturation and blood pressure.

Objective 1:

To determine the appropriate measurements and feasible packaging for the proposed device. 
Objective 2:

The device should have the following features:

- Can resist impact.

- Can be applied rapidly by staff.

- Cannot be easily removed by the patient.

- Does not cause discomfort.

- Does not have any accessible or visible wires.

- Cannot be used for destructive or aggressive purposes by the patient.

Objective 3:

All the sensor data should be transmitted wirelessly to a central monitoring station.

Objective 4:

To develop a central monitoring station which includes a GUI that can assist the psychiatrist and medical staff with the monitoring of the vital signs of the patient. This central monitoring station should continuously receive data from the device.

\subsection{Thesis Overview}

A literature study is presented in Chapter 2 where the different sensors (blood pressure, ECG, respiratory rate and oxygen saturation) are discussed. The different wireless monitoring devices on the market and relative patents will also be discussed. A mechanical concept will be developed and selected using an evaluation matrix in Chapter 3, while the chosen concept will be discussed in detail in Chapters 4 and 5. The central monitoring software with GUI and signal processing techniques will be shown in Chapter 6. Chapter 7 will discuss the laboratory testing setup together with results obtained from these tests. The report concludes in Chapter 8 with the evaluation of the system functionality and future recommendations. 


\section{CHAPTER 2}

\section{LITERATURE REVIEW AND RATIONALE}

This chapter only presents the necessary general background, although a comprehensive literature study was undertaken. In Chapter 2.1 a background explanation of each of the following vital signs (ECG, respiratory sounds, blood pressure and oxygen saturation) will be given. Similar ambulatory commercial health monitoring devices are discussed and evaluated as to why they cannot be used in a psychiatric environment.

\subsection{Vital Sign Background}

\subsubsection{Electrocardiogram (ECG)}

The ECG is commonly used in medicine due to the diagnostic information that can be extracted from the signal such as the pathophysiological condition of the heart. An ECG measurement is the electrical activity of the heart measured on the surface of the patient. The first observer was Waller in 1889 that used a capillary electrometer to measure the ECG [4]. The recording of an ECG strip is done with two electrodes. The amplitude differential between these two electrodes is used. Each of the differential electrode pair is know as a lead. Different lead setups can be used to measure the different heart activities. A 12-lead system is shown in Figure 2-1 [5].

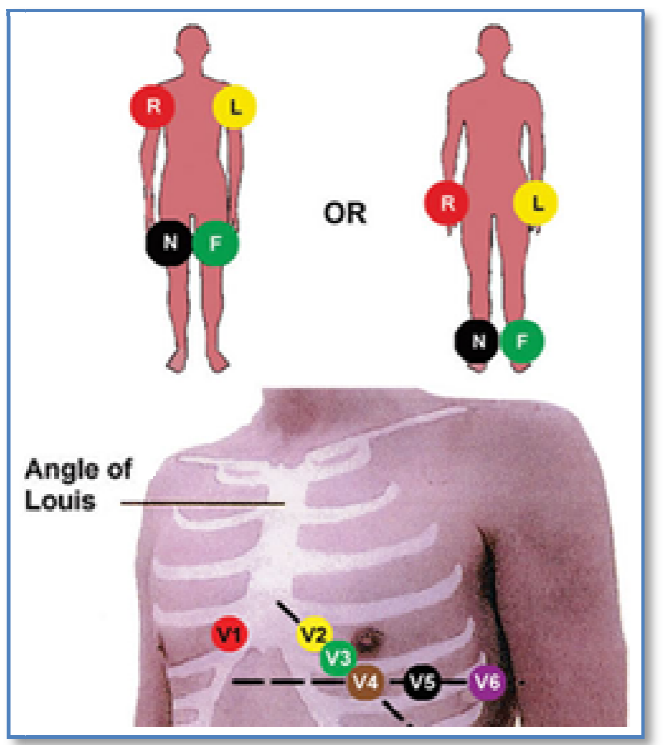

Figure 2-1 12-lead ECG electrode placement [6] 
There are various subsets of 12-lead ECG systems. In ambulatory measurements two or three lead ECG electrodes are employed. A standard two lead ECG measurement strip on the chest of the patient will yield the ECG trace shown in Figure 2-2 [7].

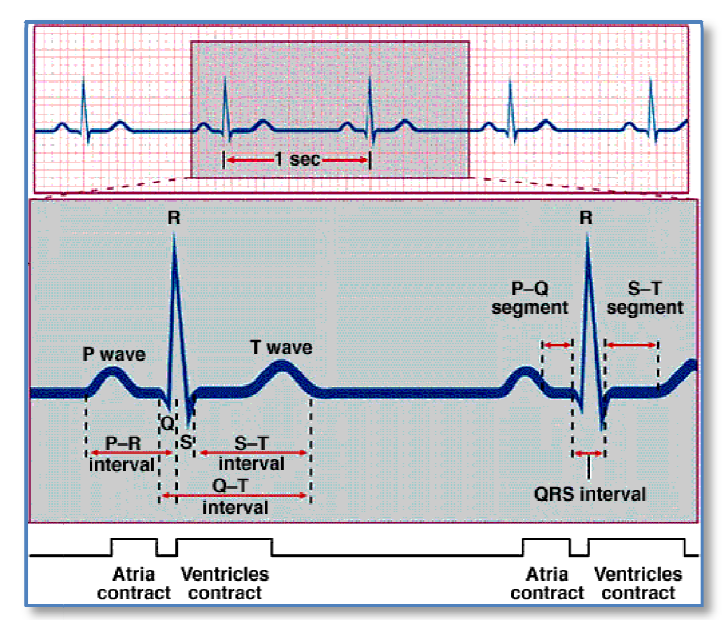

Figure 2-2 ECG rhythm strip [7]

\subsubsection{Respiratory Monitor}

\section{Background}

Breathing or ventilation is classified as a mechanical process where air is moved into the lung known as inspiration and out of the lung knows as expiration. The normal breathing rate of an adult is between 15 and 20 breaths per minute [8]. There are two sub classifications of breathing (quiet breathing or forced breathing). The amount of air that moved in and out of the lung classifies the type of breathing to one of these two. In quiet breathing only the inhalation requires muscle contraction, and with forced breathing the inhalation and exhalation require muscle contraction. With forced breathing the accessory muscles and the internal intercostal muscles together with the abdominal muscles are used [9].

\section{Respiratory Rate Monitoring}

In medical situations it is often necessary to know the patient's respiratory rate. To measure respiratory rate, different measurement techniques can be used. One such technique is using the timing characteristics of the heart sounds to calculate respiratory rate [10]. Another more feasible and less complicated method is the measurement of respiratory sounds using a digital stethoscope. Filters should be implemented to filter all the heart and ambient noise from the recorded sound signal to leave only some noise and respiratory sounds [11]. Respiratory rate can also be calculated using a piezo-electric respiration transducer which generates a signal in 
response to changes in thoracic circumference associated with respiration [12]. The transducer is in the form of a strap that is placed around the chest of the patient.

\subsubsection{Blood Pressure}

Blood pressure is related to the force exerted against the vascular walls by the blood. This is caused by the push exerted by cardiac contraction and the elasticity of the relevant vessel walls. There are two pressure readings in blood pressure: the diastolic pressure and the systolic pressure. The diastolic pressure is during the ventricular diastole and the systolic pressure is during the ventricular systole. Blood pressure used in medical terminology refers to arterial blood pressure. The standard arterial blood pressure for a healthy adult will be approximately 120 (systolic) / 80 (diastolic) [9]. Figure 2-3 shows the pressures related to the circulatory system [13].

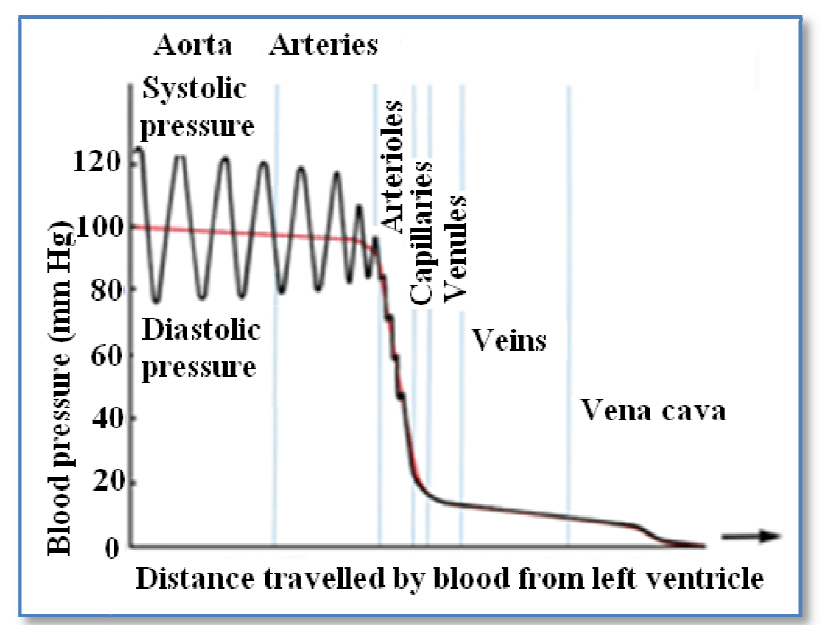

Figure 2-3 Pressures in the circulatory system [13]

\subsubsection{Oxygen Saturation}

Oxygen saturation is the relative amount of oxygen carried by the hemoglobin in the erythrocytes. A pulse oximeter based on oximetry is used to measure oxygen saturation. Oximetry is based on spectrophotometric measurements of changes in the colour of blood, in particular the relative concentration of a hemoprotein composed of globin and heme $(\mathrm{Hb})$ and microcirculatory hemoglobin oxygen saturation $\left(\mathrm{HbO}_{2}\right)$ since the characteristic colour of deoxygenated blood is blue, whereas fully oxygenated blood has a bright red colour. Therefore two specific wavelengths are used: red spectrum (around $660 \mathrm{~nm}$ ) where there is a large difference in light absorbance between $\mathrm{Hb}$ and $\mathrm{HbO}_{2}$ and infrared (IR) spectrum (around $910 \mathrm{~nm}$ ) where the absorbance of $\mathrm{Hb}$ is slightly less than that of $\mathrm{HbO}_{2}$. Figure 2-4 [8] show the optical absorption spectra of blood in the near-infrared and visible wavelength regions [8]. 


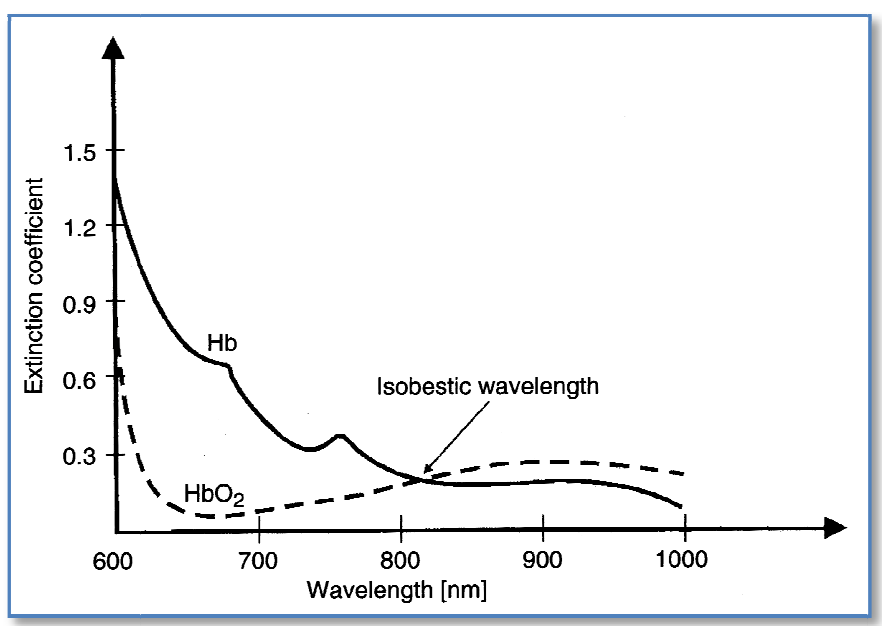

Figure 2-4 Optical properties of $\mathrm{Hb}$ and $\mathrm{HbO2}$ [8]

Two modes of operation are used for pulse oximetry, namely transmission and reflectance. In the transmission mode the optical sensor is placed across a translucent finger tip, ear lobe or toe of the patient. In the reflectance mode, the LEDs and photo detector are placed back to back on a flat surface, next to each other. The most common method used is the transmission mode, because it yields the best results. The reflectance mode method has a few problems that influence the implementation of this method.

\subsection{Portable (Ambulatory) Health Monitoring Devices}

In this section different commercial products and inventions related to ambulatory vital sign monitoring will be discussed. There are many separate commercial monitors to monitor only one vital sign, such as ECG monitors, oxygen saturation monitors and blood pressure monitors (refer to Appendix A2). The best design will be to combine these single monitoring devices into one small monitoring package that measures more than one vital sign. Therefore a few more ambulatory monitoring devices that combine some of these measurements will be explained in Chapter 2.2.1.

\subsubsection{Smart Vest Monitor [14]}

The continuous monitor smart vest developed by Pandian et al. [14] is a wearable and washable shirt with the sensors attached inside the shirt. This device connects to firmware that continuously measures the vital sign sensor data. The system has a global positioning system (GPS) integrated with the microcontroller and wireless communication. The six physiological variables that the smart vest can monitor are ECG, photoplethysmogram (PPG), body temperature, blood pressure, galvanic skin response (GSR) and heart rate. This device has all the sensors integrated in the one vest, depicted in Figure 2-5 [14]. The problem why this type of device cannot be used 
in a psychotic patient environment is the amount of wires used for the PPG sensor (to calculate blood pressure) and the GSR sensor, as well as the size of the jacket. The cost to develop different size jackets to fit small, medium and large patients will be too high [14].

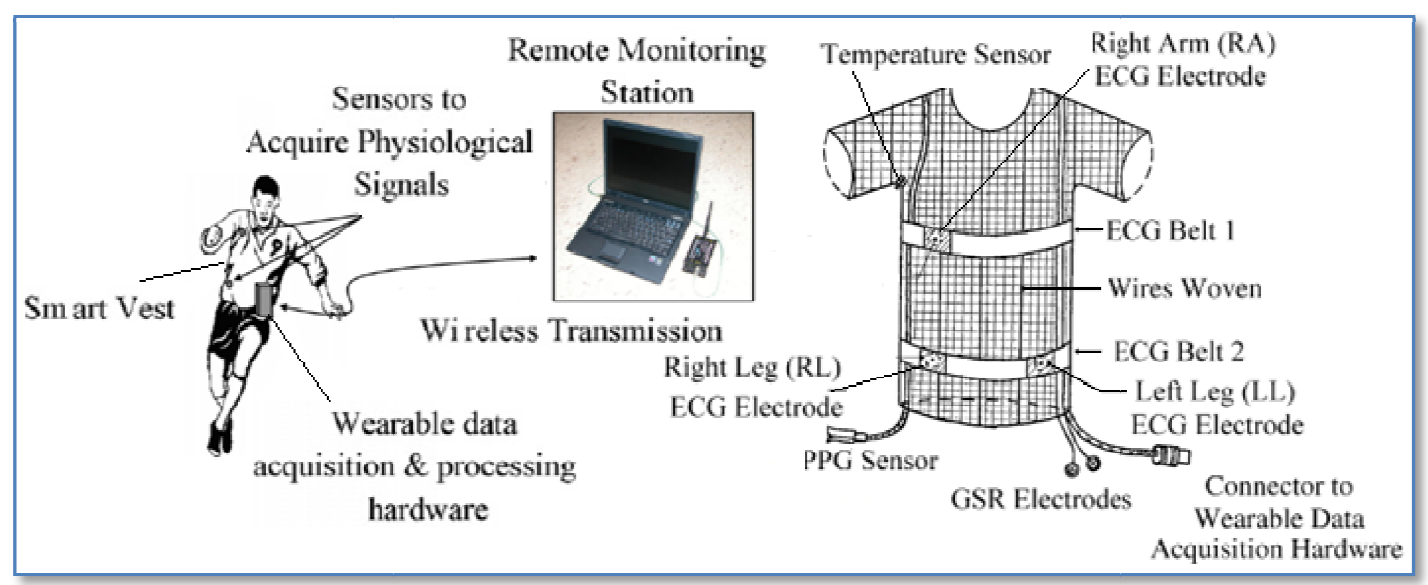

Figure 2-5 Smart vest monitor system [14]

\subsubsection{SOMNOcheck [15]}

The following parameters are recorded using the SOMNOcheck system from Weinmann medical technology [15]: oxygen saturation, pulse rate, respiratory flow (via thermistors or pressure sensor), snoring sounds (via microphone or pressure sensor), BiLevel pressure or SmartPAP pressure, and the patient's position. Figure 2-6 shows the SOMNOcheck system [15]. The use of wires makes it impractical for use in a psychiatric environment.

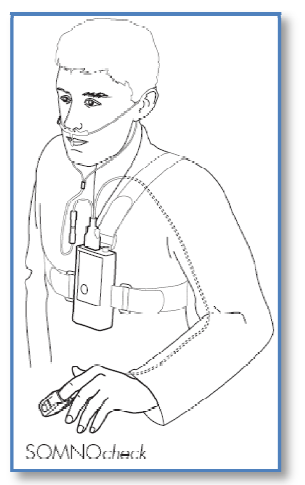

Figure 2-6 SOMNOcheck device attached to patient [15] 


\subsubsection{LifeGuard}

The LifeGuard device developed by NASA is worn on the body as shown in Figure 2-7 [16]. It has several sensors connected with wires to the main processing unit. The device can measure ECG (two channels), respiration, movement activity (3-axis acceleration), temperature (skin or ambient), heart rate, pulse oximetry, and diastolic and systolic blood pressure. The device can monitor and collect these variables for up to nine hours and stream to a base station using a Bluetooth link [16]. This device is well-developed but needs to be changed for use in a psychiatric environment, due to the large number of wires used to connect to all the different sensors.

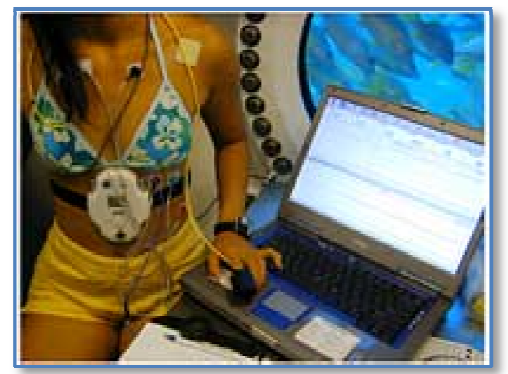

Figure 2-7 NASA “CPOD” wireless LifeGuard monitor [16]

\subsubsection{Programmable Wireless Electrode System for Medical Monitoring [17]}

This wireless ECG electrode system (USA patent) is developed by Richard et al. [17]. Each electrode of the ECG system is connected separately to a wireless central base unit using remote programmable transceivers connected to each electrode. The base unit controls the electrodes of the ECG system using a defined communication protocol. The data measured with this system are then displayed on a monitoring screen as illustrated with the block diagram in Figure 2-8 [17]. With this patent the physical size of the ECG device (only electrodes) attached the patient is minimised.

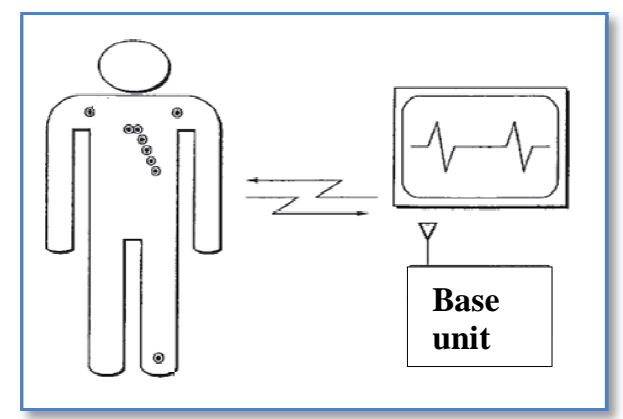

Figure 2-8 Block diagram of system [17] 


\subsubsection{Vital Sign Remote Monitoring Device [18]}

This vital sign monitoring device is used to monitor hospitalised patients remotely. This USA patent device developed by Money et al. [18] is incorporated into a portable housing and is wearable by an ambulatory patient. This device can connect external ECG, temperature, oxygen saturation $\left(\mathrm{S}_{\mathrm{a}} \mathrm{O}_{2}\right)$ and respiratory sensors to the remote monitoring device. The connections for these sensors are shown in Figure 2-9 [18]. These analogue and digital signals are then transmitted to a central monitoring station via a radio frequency (RF) transmitter and receiver. Figure 2-9 shows the block diagram of the vital sign remote monitoring device [18].

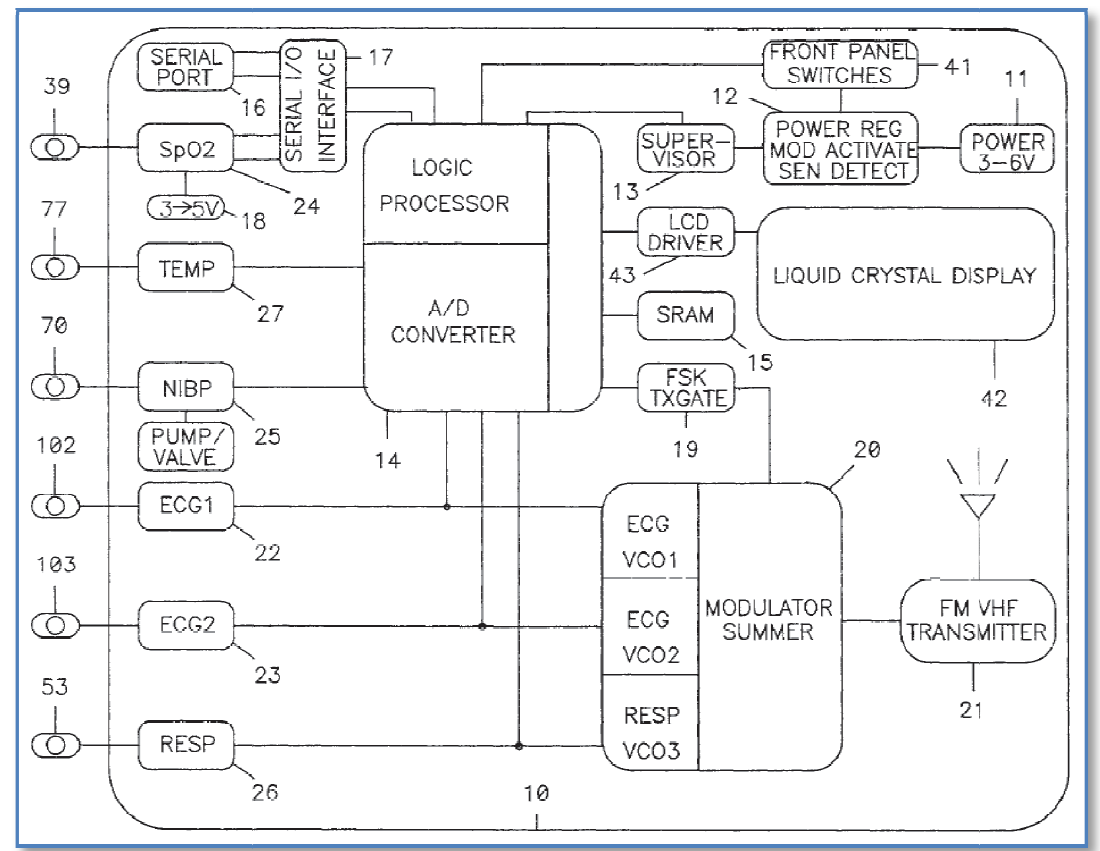

Figure 2-9 Block diagram of patent system [18]

\subsubsection{Wireless Medical Diagnosis and Monitoring Equipment [19]}

This USA patent describes a medical diagnosis and monitoring system developed by Besson et al. [19], having at least one sensor for detecting an electrical, physical, chemical, or a biological property of a patient [19]. This includes ECG, respiration, oxygen saturation, temperature, perspiration, and others. A digital-to-analogue converter (DAC) coupled to the sensor and a digital transmitter and receiver for wireless communication to the central monitoring station are implemented [19]. The possible locations of electrode placements for measuring different vital sings are shown in Figure 2-10 [19]. 


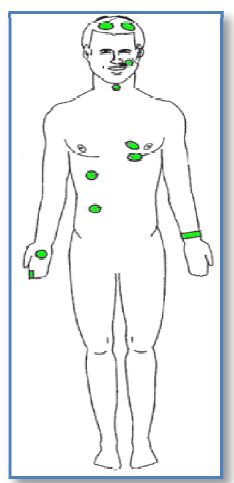

Figure 2-10 Arrangement of electrodes placement on patient [19]

\subsubsection{Method and Apparatus for Monitoring Physiological Parameters of a Living Subject [20]}

This USA patent is a wireless vital sign monitor system developed by Gallant \& Markle [20]. Although the sensors are placed in fixed chair design shown in Figure 2-11 [20], the concept to monitor vital signs and send it wirelessly to a central monitor station is illustrated with this patent. This design cannot be used in a psychiatric patient environment, but shows which vital signs are typically monitored. The wireless protocol used in this patent is an RF link, as illustrated in Figure 2-11 [20].

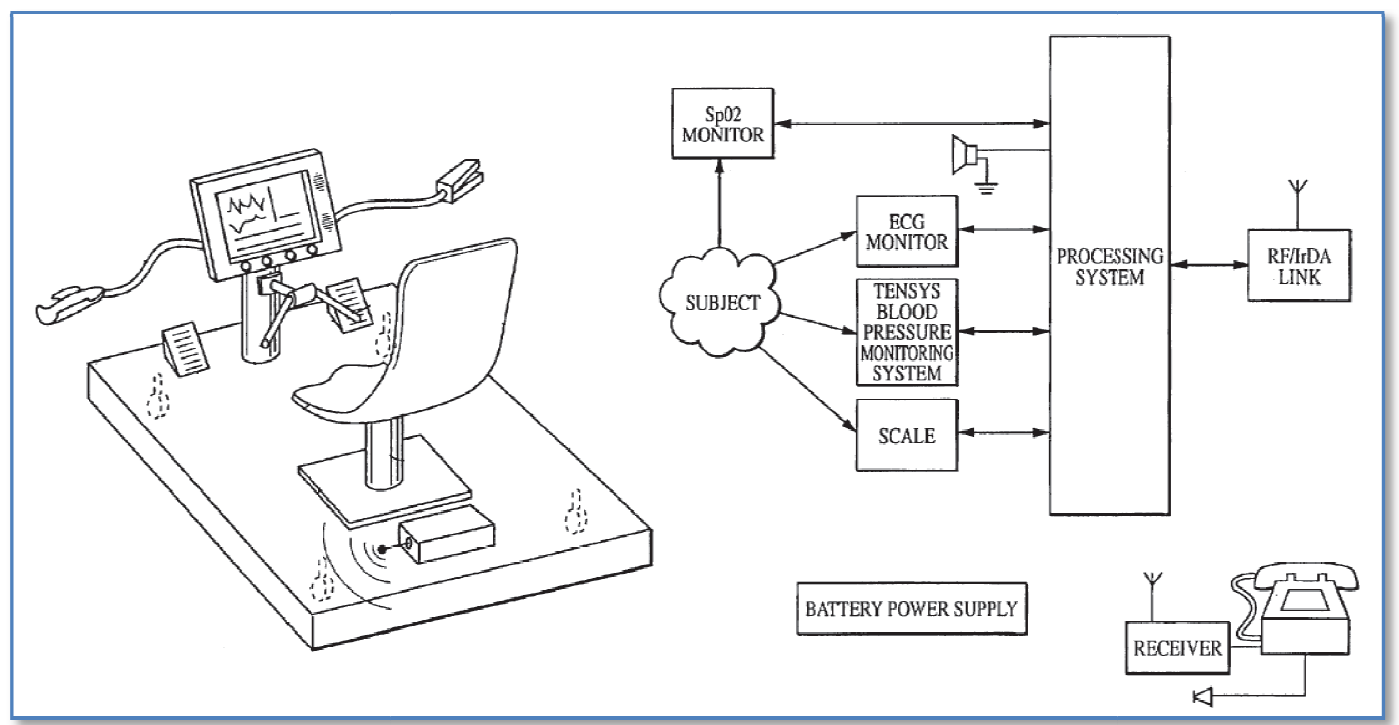

Figure 2-11 Physiological parameters monitoring system [20] 
The device can continuously monitor blood pressure, heart rate (using ECG), oxygen saturation and the weight of the patient. The device comprises of a portable monitoring station which is kept in communication via a wireless interface with a remote medical facility. The patient can be monitored remotely via video, audio and sensor data by a medical practitioner as shown in Figure 2-12 [20].

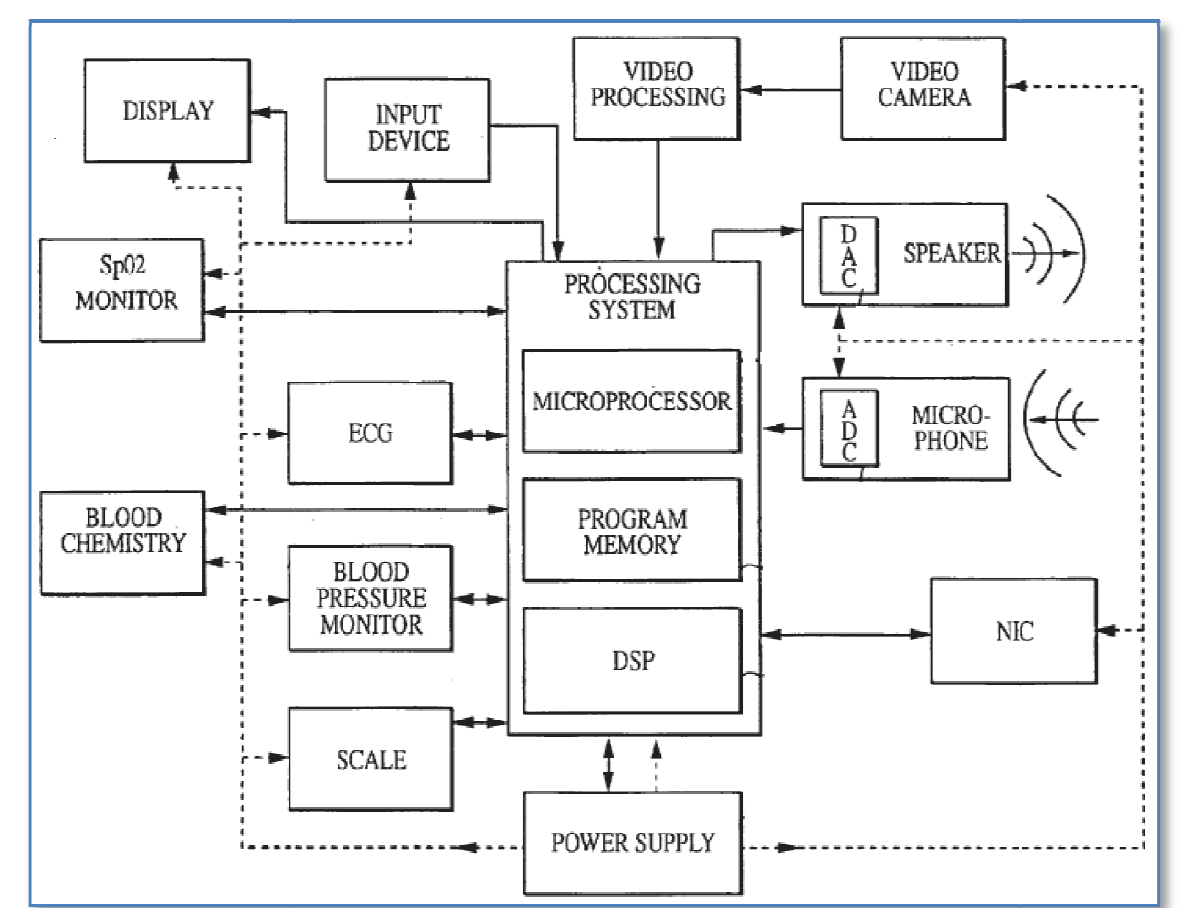

Figure 2-12 Block diagram of physiological parameters monitoring system [20]

\subsubsection{Device System and Method for Monitoring Vital Signs [21]}

This USA patent developed by Banet \& Visser [21] is a vital sign monitoring device and is shown in Figure 2-13 [21]. This device is capable of determining when a user's wrist is at rest, using an accelerometer (ACC) sensor disposed within a wrist module that is attached to the user's wrist. When at rest, the device monitors the vital signs to determine a plurality of vital signs of the user. The vital sign monitor comprises of a light source and photo detector in communication with a pulse oximetry circuit. The oxygen saturation is measured and a time-dependent waveform is used with a light emitting diode (LED) and photodiode (PD) to detect continuous blood pressure (BP). All of the data are sent wirelessly to a central monitoring station [21]. 


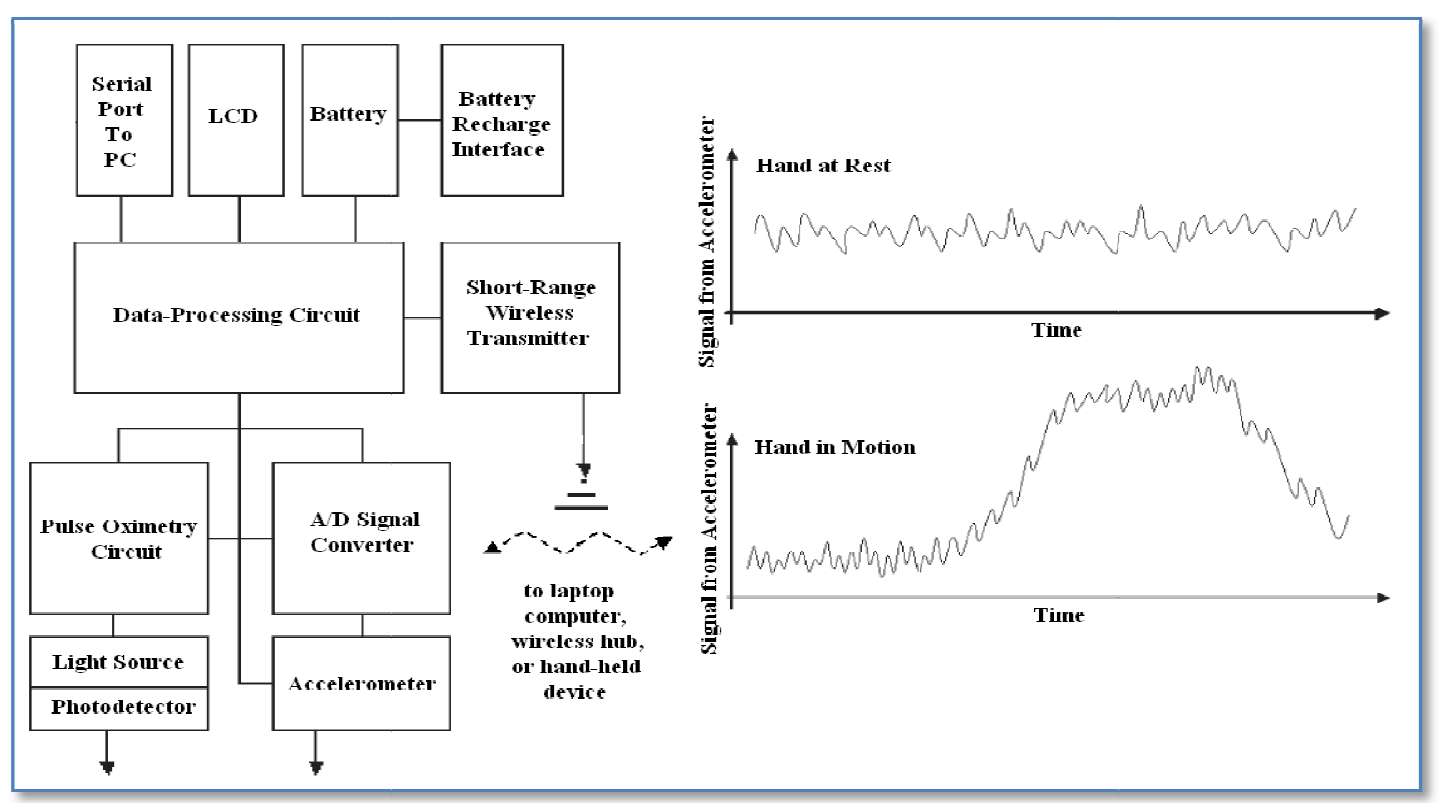

Figure 2-13 Block diagram and accelerometer output with hand at rest and in motion [21]

\subsubsection{Wearable Health Care System for Vital Sign Monitoring [22]}

A wearable health care system for vital signs monitoring was developed by Paradiso [22]. This design is based on the use of standard industrial processes to realize the sensing elements. An innovative system named WEALTHY is developed, were fiber and yarn form is used as a smart material. A wide range of electro physical properties (piezoresistive, conducting, etc.) are integrated and used as basic elements to be woven or knitted into fabric form. The respiratory rate and heart rate is measured with this smart material. A 3G (GPRS/UMTS) wireless communication protocol is used to send the vital signs to a remote monitoring station [22].

\subsubsection{A WBAN (Wireless Body Area Networks) System for Ambulatory Monitoring of Physical Activity and Health Status [23]}

A WBAN system for ambulatory monitoring of physical activity and health status is developed by Jovanov et al. [23]. A standard platform is developed with common off-the-shelf wireless sensor platforms like a ZigBee-compliant radio interface, an ultra low-power microcontroller and accelerometer circuits. These standard accelerometers are used for motion monitoring and a bioamplifier for ECG monitoring. The software modules for on-board processing, communication, and network synchronization have been developed. The objective of Jovanov [23] is to 
standardise the ambulatory monitoring platforms with reusable sensors and wireless communication protocols for future developments in this research field [23].

\subsubsection{AMON: A Wearable Multiparameter Medical Monitoring and Alert System [24]}

The AMON system developed by Anliker et al. [24] is a wrist worn remote monitoring device, as shown in Figure 2-14 [24]. This paper stated that the results obtained by measurements of a single-lead ECG, reflectance oxygen saturation and skin temperature at the wrist location were not good enough to be useful for monitoring. Blood pressure was measured using a pressure cuff concept. All the measured data was send to an online medical mission control (MCC) station using a global system for mobile (GSM) communication protocol [24].

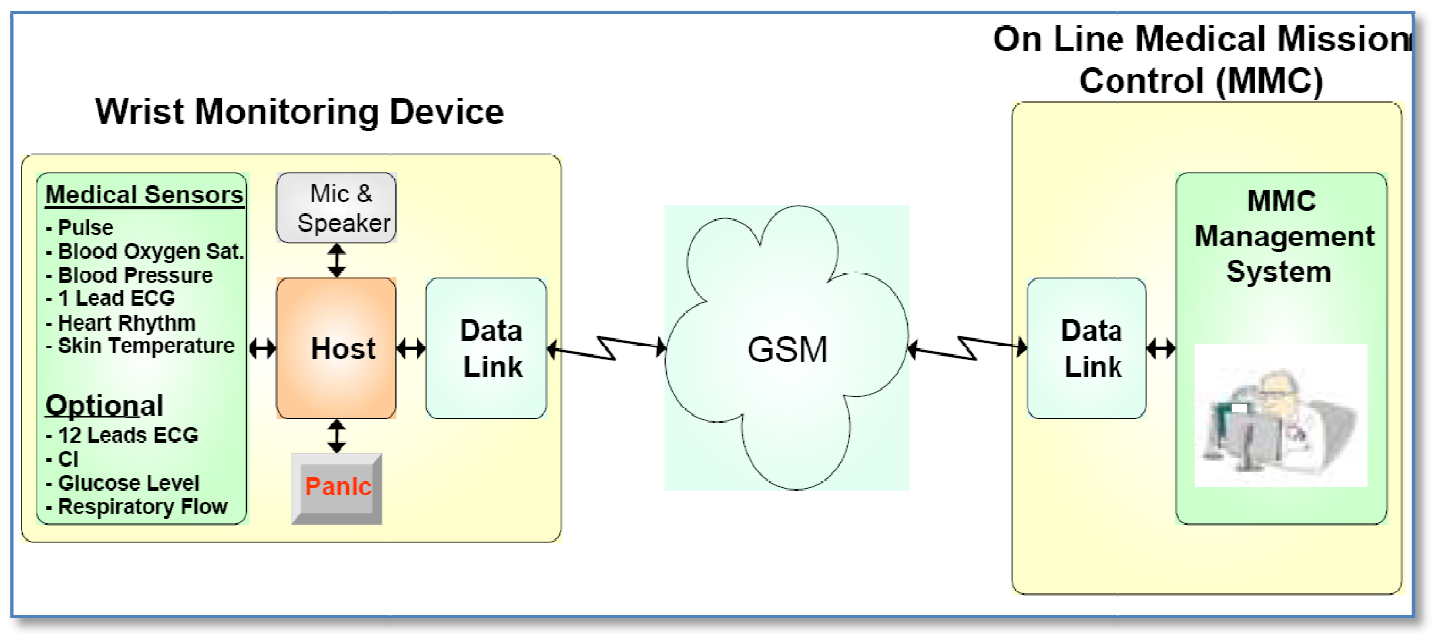

Figure 2-14 Wrist worn medical device with GSM/UMTS link to telemedicine centre [24]

\subsection{Conclusion}

As can be seen from the research, the development of a small wireless device that can resist impact and measure the vital signs of a psychotic patient is needed. From the literature review, the mentioned devices can measure all the needed vital signs but are not suitable for a psychotic patient environment. The following chapter will continue to investigate what type of vital signs can be measured while considering the mechanical limitations that a psychotic patient poses. Appendices A1 and A2 present further literature on continuous blood pressure monitoring and commercial vital sign monitoring devices. 


\section{CHAPTER 3}

\section{CONCEPT DEVELOPMENT}

The implementation of the mechanical device is limited by the project objectives stated in Chapter 1.2. The use of the device on acute psychotic patients limits the positioning of the device on the patient due to the aggressive nature of such a patient. Using these mechanical limitations the measurement of certain vital signs at a particular location is also limited by the physiology at those locations, together with the electronic design limitations of different measuring techniques. All of these factors must be considered to choose a specific practical location of the device on the patient.

\subsection{Proposed Mechanical Designs}

Background research indicated that variables such as oxygen saturation, ECG, respiratory sounds, temperature, heart rate and possibly the blood pressure of the patient can be measured. Four possible positions for the device were identified using the project objectives, physiology and electronic limitations. The four concepts (safety cuff, safety glove, safety shoe and dorsal mounted) are compared in an evaluation matrix, shown in Table 3-1. The evaluation (decision) matrix method proposed by Ullman [25], evaluates each concept to specific requirement set. A weight is given for each requirement relative to the projects requirements. From this matrix (shown in Table 3-1) an independent score is calculated for each concept.

Four main steps are used to calculate this matrix:

1. Calculate criteria for comparison from specifications.

2. Calculate relative important weights for each of the requirement specifications.

3. Evaluate the requirements to each of the concepts.

4. Compute a weighted result score.

From the results obtained in Table 3-1 it is clear that the dorsal mounted concept scored 721 points compared to the safety glove concepts score of 540. The score of 721 was the best and therefore the dorsal mounted device will be the best concept to be implemented for the requirements set for this project.

Each of the four concepts discussed in Table 3-1 will be discussed briefly in this section. 
Table 3-1 Evaluation matrix

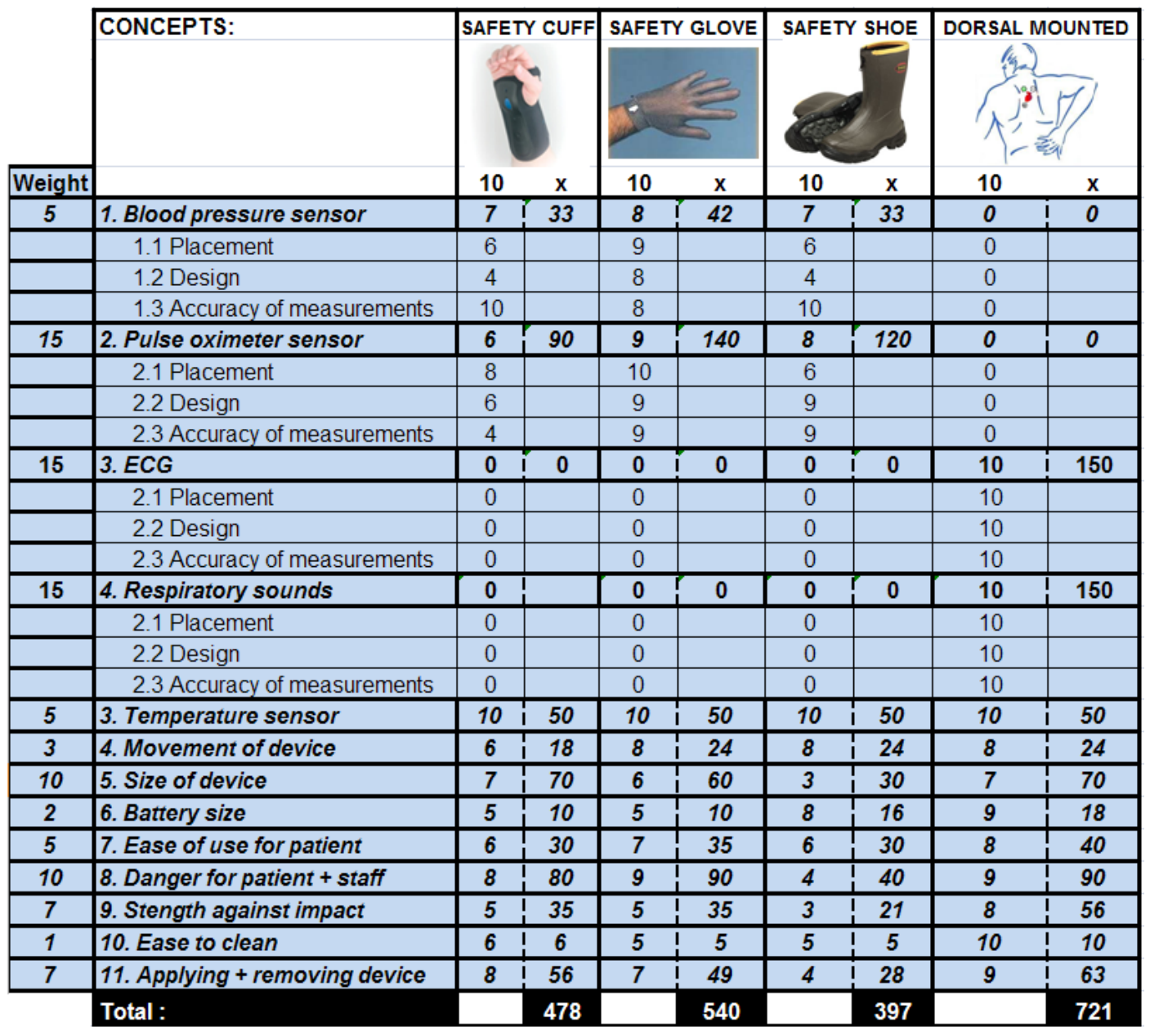

\subsection{Safety Cuff Concept}

In the safety cuff concept, oxygen saturation, blood pressure, temperature and physical movement can be measured. The implementations of the temperature and movement sensors are simple compared to the implementation of the pulse oximeter and blood pressure sensor. The pulse oximeter can be implemented using the reflectance probe method. This method places the red and infrared light and the photodiode next to each other. The scattered reflectance light from the oxygenated blood is measured by the photodiode. Figure 3-1 shows the implementation diagram of the reflectance probe method and placement of diodes. 


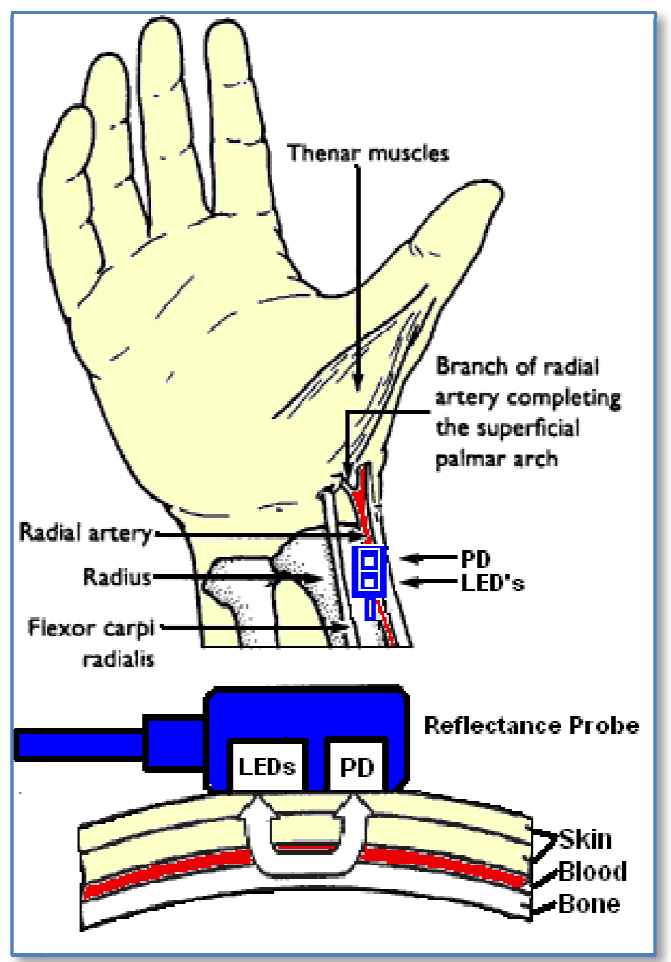

Figure 3-1 Reflectance probe placement over radial artery on wrist

The LEDs and the photodiode are placed on the wrist of the patient over the radial artery. The problem with this method lies with the physical movement of the patient. When the cuff moves on the wrist, the LEDs and photodiode will not be aligned over the radial artery, providing a weak pulsating signal for the saturation calculation. When the device moves more than $1 \mathrm{~cm}$ around the arm, the pulsating signal will disappear and no saturation reading would be possible. This makes it impractical for the purposes of this project to measure saturation using the reflectance probe method as a psychotic patient has the tendency to be aggressive, and move a lot with his hands which will move the cuff device around the arm.

Only periodical blood pressure measurements can be implemented by using a cuffbased design where a small cuff around the wrist inside the monitoring device could be inflated with an air pump. The problem with this design is the size of the device that would be placed around the wrist. The battery required to drive the air pump that will inflate the cuff will be large and heavy. Figure 3-2 shows a commercial blood pressure wrist cuff that could be placed around the wrist. The problem is that the device should be kept as small as possible to ensure that the psychotic patient will not be irritated by the device, and it must resist impact should the patient attempt to destroy the device. 


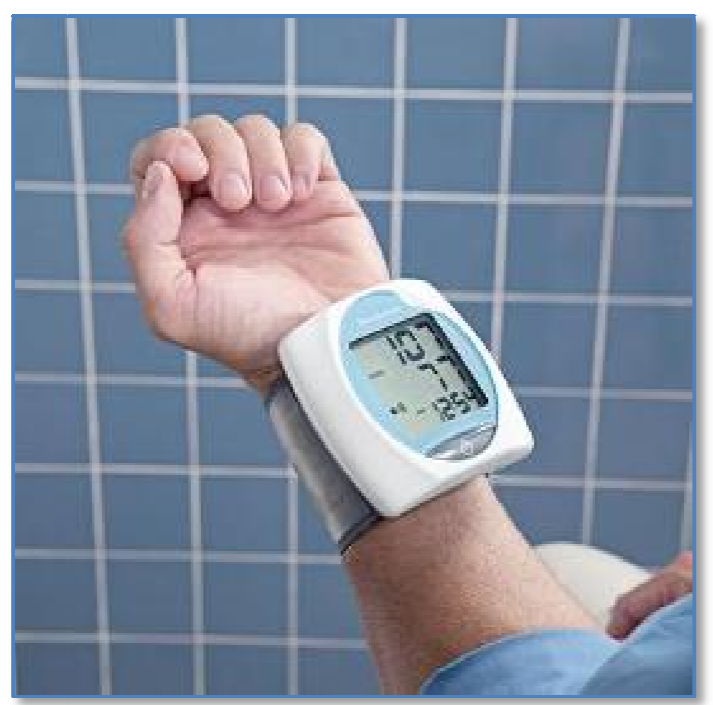

Figure 3-2 Commercial wrist blood pressure monitor

\subsection{Safety Glove Concept}

In the safety glove concept oxygen saturation, temperature, blood pressure and motion of the patient can be measured.

The implementation of a normal pulse oximeter can be done by using a finger clip from a commercial pulse oximeter probe. The main problem is to cover the wires running from the finger probe to the monitoring electronics worn at the wrist of the patient. A rubber glove will be used to cover and protect the finger probe and the connection wires, for example as shown in Figure 3-3.

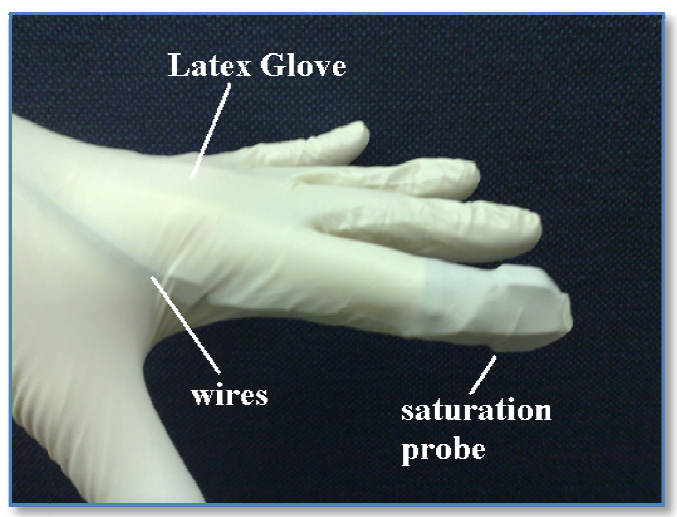

Figure 3-3 Saturation probe and wires protected 
The implementation of the wireless connection together with the microcontroller circuit that measures and calculates the heart rate and saturation ratio value can be done using a small protective housing. This housing can be covered with the same glove that covers the wires and saturation probe and should be easy to install.

To measure blood pressure non-invasively over long periods of time is a difficult task. The conventional methods of measuring blood pressure cannot be used due to the size of the cuff, the discomfort for the patient, and the amount of battery usage to inflate the pressure cuff. Literature studies indicated that Yang et al. [26] developed a new method to estimate blood pressure non-invasively. The design uses a PPG sensor together with an impedance plethysmograph (EIP) [27] sensor to estimate blood pressure using a hemodynamic model of the systemic circulation and a Kalman filter.

A mathematical model of the arterial blood flow is derived and changed into a statespace representation. In the modelling, a precise hemodynamic model for the arterial segment, on which sensors are located, is derived. These models can then be joined with relatively simplified models of the upstream and the downstream arterial flows to obtain an entire arterial stream. Then a Kalman filter is designed based on this model. It can be shown that the internal variables, such as the arterial blood pressure in the arterial segment, can be estimated based on these measurements, even though the observability condition of the whole system may not be met [26].

Figure 3-4 shows a diagram of the estimation process used to estimate the blood pressure from the measured signals [26].

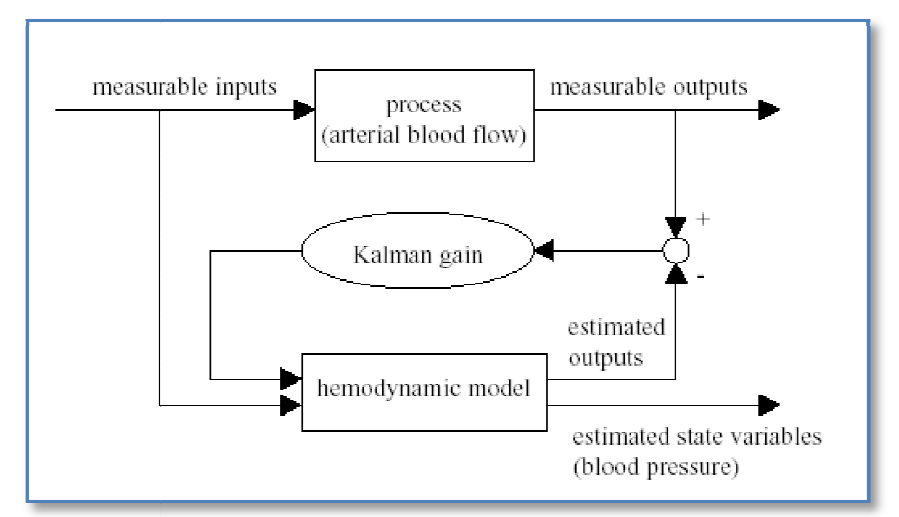

Figure 3-4 A schematic diagram for estimating blood pressure [26]

This method of measuring blood pressure was implemented and tested but without any usable results. The implementation of the PPG was done and results were obtained as expected. The problem was to implement a working EIP sensor. Figure 3-5 shows the testing setup and implementation of the ring sensors on the left middle 
finger. It is expected to measure a modulated $50 \mathrm{kHz}$ signal. The modulation should be a result of the pulsation of the blood volume through the digital artery. However, the change in impedance was so small that after peak detection circuit only a direct current (DC) value was measured. The measurement of blood pressure was left for future research and not used further in this project due to time constraints. For more information on the implementation of this method, refer to Appendix A1.

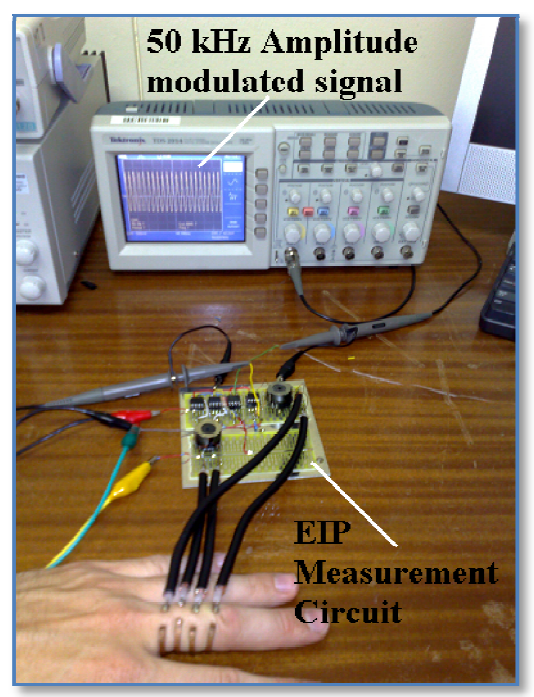

Figure 3-5 EIP Circuit with modulated $50 \mathrm{kHz}$ sinusoidal signal

\subsection{Safety Shoe Concept}

In the safety shoe concept, oxygen saturation, skin temperature and patient motion can be measured.

The measurement of the oxygen saturation can be done by placing a probe on a toe of the patient. The microcontroller electronics, together with the battery supply, can be inserted into the sole of the boot. The advantage of the shoe device concept is the space available for the battery and the electronics, and the mechanical protection of the wires and electronics. The disadvantage of this design is the safety of the clinician on duty. The patient can use the boot to kick the clinician and will put the clinician in danger. From Table 3-1 it is clear that the boot device concept score was the worst, due to the safety for the medical personnel and the limitation of the measurements. Hence it was not further considered as a viable concept. 


\section{CONCEPT DEVELOPMENT}

\subsection{Dorsal Mounted Concept}

In the dorsal mounted concept, ECG, respiratory sounds, skin temperature and movement can be measured.

The dorsal mounted device can be attached between the left and right scapula of the patient, as shown on Figure 3-6.

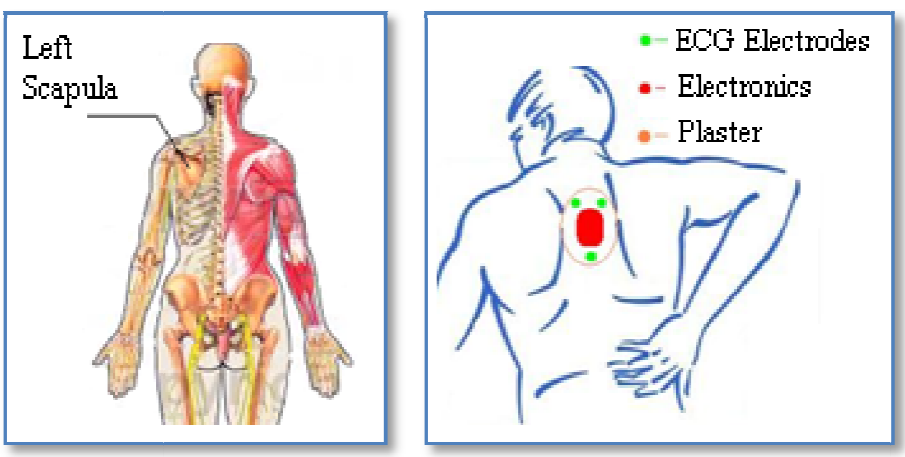

Figure 3-6 Placement of dorsal mounted device

Most patients cannot stretch their hands very far behind their backs, ensuring that the device is safe from an aggressive patient. The device cannot be used to injure the patient or the clinician on duty. It will be very easy to attach the device by using a large adhesive plaster, and it will be practically impossible for the patient to remove or damage the device. These features also best meet the primary objectives of the project.

From the measured ECG waveform further analysis can be made such as heart rate variability (HRV) and detection of premature ventricular contraction (PVC) and atrial fibrillation.

\subsection{Chapter Summary}

The outcome of the evaluation matrix from Table 3-1 shows that the dorsal mounted device is the most promising concept, followed by the safety glove. After a meeting with Prof. JF Coetzee (Anaesthetist) and Dr. G Winkler (Psychiatrist), it was decided that ECG and oxygen saturation will be needed to monitor a psychotic patient efficiently. To comply with this request the project will be divided into two parts, the dorsal mounted device and the safety glove device. It is not possible to measure oxygen saturation on the back of the patient. Therefore the measurement of oxygen saturation should be done with a separate device, using the safety glove device. 
Therefore, the dorsal mounted device and the safety glove device was further developed in this project. The dorsal mounted device will implement ECG, respiratory sounds, skin temperature and motion sensors. The safety glove device will implement the pulse oximeter to measure oxygen saturation and patient motion using an accelerometer. These two devices communicate to one central monitoring station using two separate Bluetooth connections to ensure a totally wireless system. The dorsal mounted device is the primary device and the safety glove device can be considered as an additional device. Chapter 4 presents the implementation of the dorsal mounted device and Chapter 5 presents the safety glove device. Figure 3-7 shows a block diagram which gives an overview of the whole system.

\subsubsection{Safety Glove System Overview}

The safety glove's controller enclosure consists of a microcontroller that controls the sampling and sending of saturation data. The power supply and Bluetooth communication connections are displayed in Figure 3-7 a). The finger clip probe located on the index finger is also part of the safety glove system but is located outside of the safety glove's enclosure.

\subsubsection{Dorsal Mounted System Overview}

The dorsal mounted device consists of a microcontroller that controls the sampling and sending of ECG, sound, skin temperature and accelerometer values. The power supply and Bluetooth communication connections are displayed in Figure 3-7 b). All the components, including the sensors and Bluetooth module will be included in one housing, as illustrated by the dorsal mounted concept part of the block diagram in Figure 3-7 b).

\subsubsection{Central Monitoring Station Overview}

The central monitoring station will include a user friendly GUI, which will wirelessly receive data from the two devices and apply filters and detection algorithms to monitor the selected vital signs. 
a)

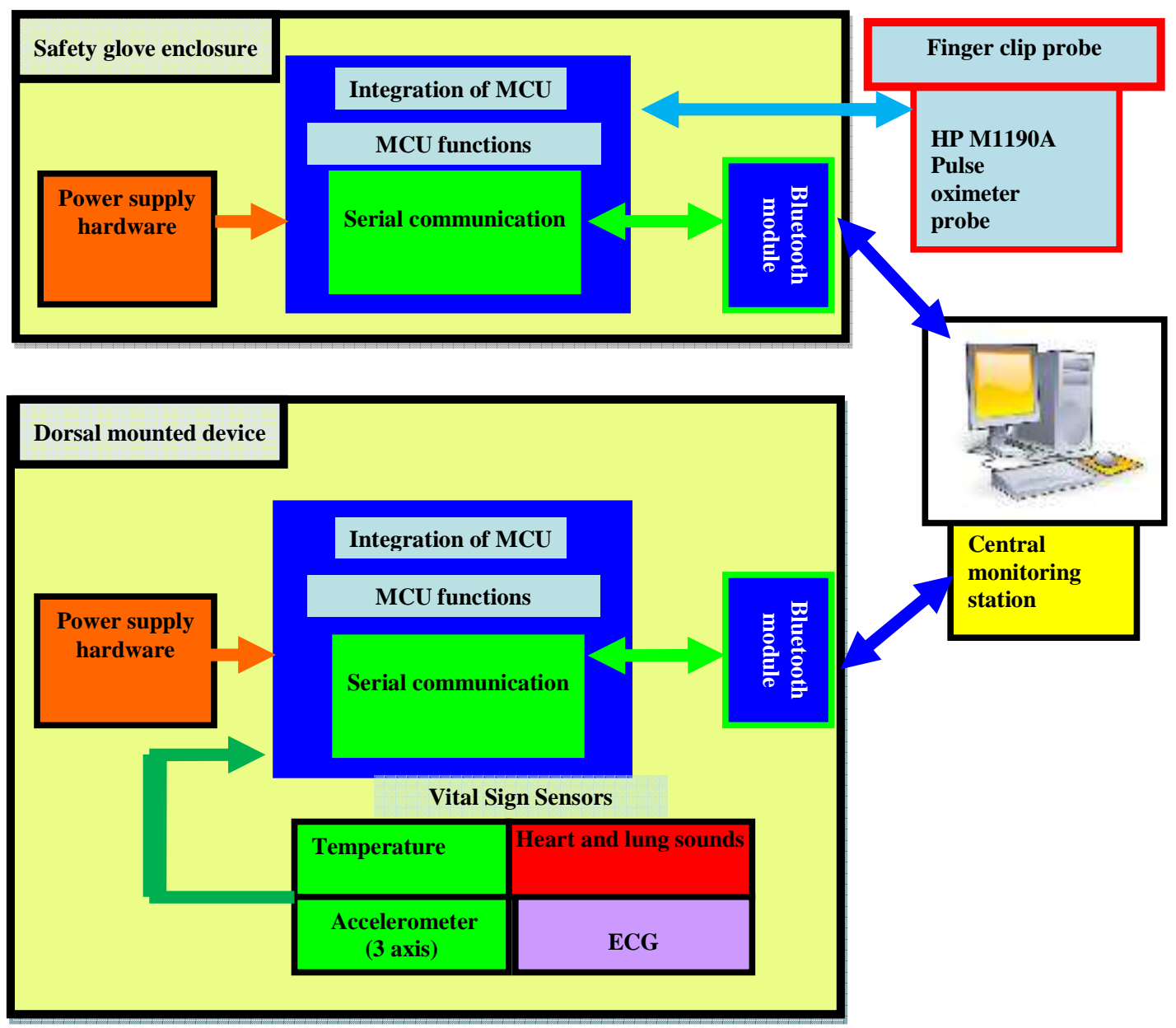

Figure 3-7 Block diagram of system 


\section{CHAPTER 4}

\section{DEVELOPMENT OF THE DORSAL MOUNTED DEVICE}

\subsection{Specifications of Dorsal Mounted Device}

The device must be mounted dorsally (on the back) of the patient. To measure variables such as ECG, heart rate, respiratory rate, movement and skin temperature, sensors must be attached to the body of the patient. To ensure that the device is as small as possible, one microcontroller is used to sample all the sensors and send this data using a dedicated Bluetooth connection. Table 4-1 shows the specifications for the battery, ECG, lung sound, temperature, accelerometer and serial communication of the dorsal mounted system. Figure 4-1 shows a picture of the developed prototype.

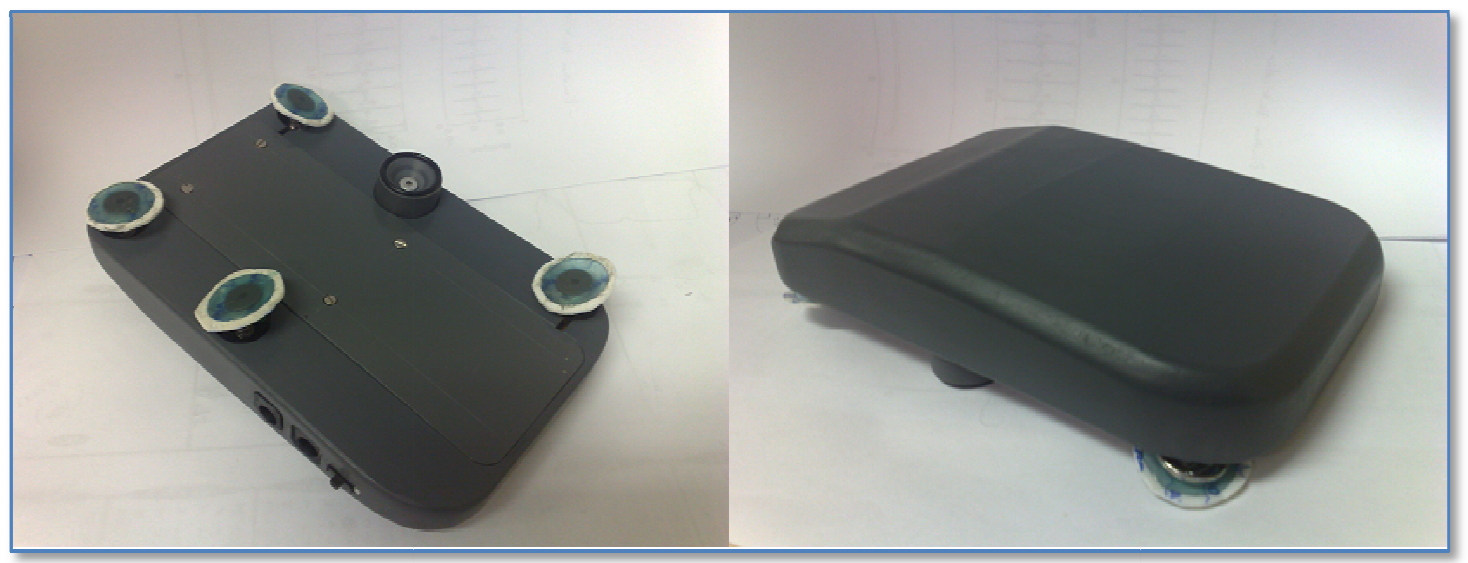

Figure 4-1 Dorsal mounted prototype 
DEVELOPMENT OF DORSAL MOUNTED DEVICE

Table 4-1 Specifications of dorsal mounted device

\begin{tabular}{|c|c|c|c|c|c|c|c|}
\hline & Power & General & $\begin{array}{l}\text { ECG } \\
\text { leads }\end{array}$ & Filters & $\begin{array}{l}\text { Signal } \\
\text { range and } \\
\text { gain }\end{array}$ & Resolution & $\begin{array}{l}\text { Sampling } \\
\text { rate }\end{array}$ \\
\hline Battery & $\begin{array}{c}\text { Two-cell } \\
\text { Li-ion } \\
3.7 \mathrm{~V} \\
\text { Recharge } \\
\text {-able } \\
\text { batteries }\end{array}$ & $\begin{array}{l}\text { More than } \\
4 \text { hours } \\
\text { battery } \\
\text { life } \\
\text { before } \\
\text { recharge }\end{array}$ & --- & --- & --- & --- & --- \\
\hline $\mathrm{ECG}$ & $\begin{array}{l}3.3 \mathrm{~V} \\
\text { single } \\
\text { supply }\end{array}$ & $\begin{array}{l}\text { CMRR: } \\
+60 \mathrm{~dB}\end{array}$ & $\begin{array}{c}2 \\
\text { lead }\end{array}$ & $\begin{array}{c}10 \mathrm{~Hz} \\
- \\
\text { Low- } \\
\text { pass }\end{array}$ & $\begin{array}{c}\text { Range: } \\
-5.0 \mathrm{mV} \text { to } \\
+5.0 \mathrm{mV} \\
\text { signals } \\
\text { Gain: } \\
+-500\end{array}$ & 8 bit & $\begin{array}{l}\text { At least } \\
500 \mathrm{~Hz} \text { or } \\
\text { more for } \\
\text { accurate } \\
\text { detection } \\
\text { of } \mathrm{R} \\
\text { peaks }\end{array}$ \\
\hline Lung sound & $\begin{array}{c}3.3 \mathrm{~V} \\
\text { single } \\
\text { supply }\end{array}$ & --- & --- & $\begin{array}{c}180 \\
\sim 380 \\
\mathrm{~Hz}- \\
\text { Band- } \\
\text { pass } \\
\text { filter }\end{array}$ & $\begin{array}{l}\text { Gain: } \\
+-300\end{array}$ & 12 bit & $\begin{array}{l}1600 \mathrm{~Hz} \\
\text { required }\end{array}$ \\
\hline Temp & $\begin{array}{l}3.3 \mathrm{~V} \\
\text { single } \\
\text { supply }\end{array}$ & $\begin{array}{l}25^{\circ} \mathrm{C} \text { to } \\
50^{\circ} \mathrm{C}\end{array}$ & --- & --- & --- & 12 bit & $\begin{array}{c}1 \mathrm{~Hz} \\
\text { required }\end{array}$ \\
\hline $\begin{array}{l}\text { ACC } \\
(3 \text { channels })\end{array}$ & $\begin{array}{l}3.3 \mathrm{~V} \\
\text { single } \\
\text { supply }\end{array}$ & $\begin{array}{l}0 \mathrm{~g} \text { to } 6 \mathrm{~g} \\
\text { for } 3 \text { axis }\end{array}$ & --- & --- & --- & 8 bit & $\begin{array}{l}100 \mathrm{~Hz} \\
\text { required }\end{array}$ \\
\hline $\begin{array}{l}\text { Serial port } \\
\text { (Bluetooth) }\end{array}$ & $\begin{array}{l}3.3 \mathrm{~V} \\
\text { single } \\
\text { supply }\end{array}$ & $\begin{array}{l}\text { Baud rate: } \\
\text { up to } \\
115.2 \mathrm{k}\end{array}$ & --- & --- & --- & --- & --- \\
\hline
\end{tabular}

\subsection{Electronic Design}

To measure the vital signs, a microcontroller with analogue to digital conversion (ADC) inputs is needed. This section will explain the main electronic components used in the prototype system. Each sensor's electronics will be discussed in detail and a final prototype printed circuit board (PCB) is presented. 


\subsubsection{Selection of Main Electronic Components}

Upon inspection of the block diagram in Figure 4-2 it is clear that a microcontroller is needed for sampling the six ADC channels at 12-bit resolution, and it must be able to send this calculated data to the Bluetooth module at a baud rate of 115200 bps. A baud rate of $115200 \mathrm{bps}$ is selected given that six ADC channels should be transmitted with a sampling rate of $1600 \mathrm{~Hz}$ each. A Bluetooth module is needed for the wireless communication. A battery supply unit which can supply at least $4.7 \mathrm{~V}$ to the $3.3 \mathrm{~V}$ voltage regulator is needed to ensure that the microcontroller, Bluetooth module and other electronic components receive a stable $3.3 \mathrm{~V}$ supply. The selection of the specific components to fulfil these requirements will be discussed below.

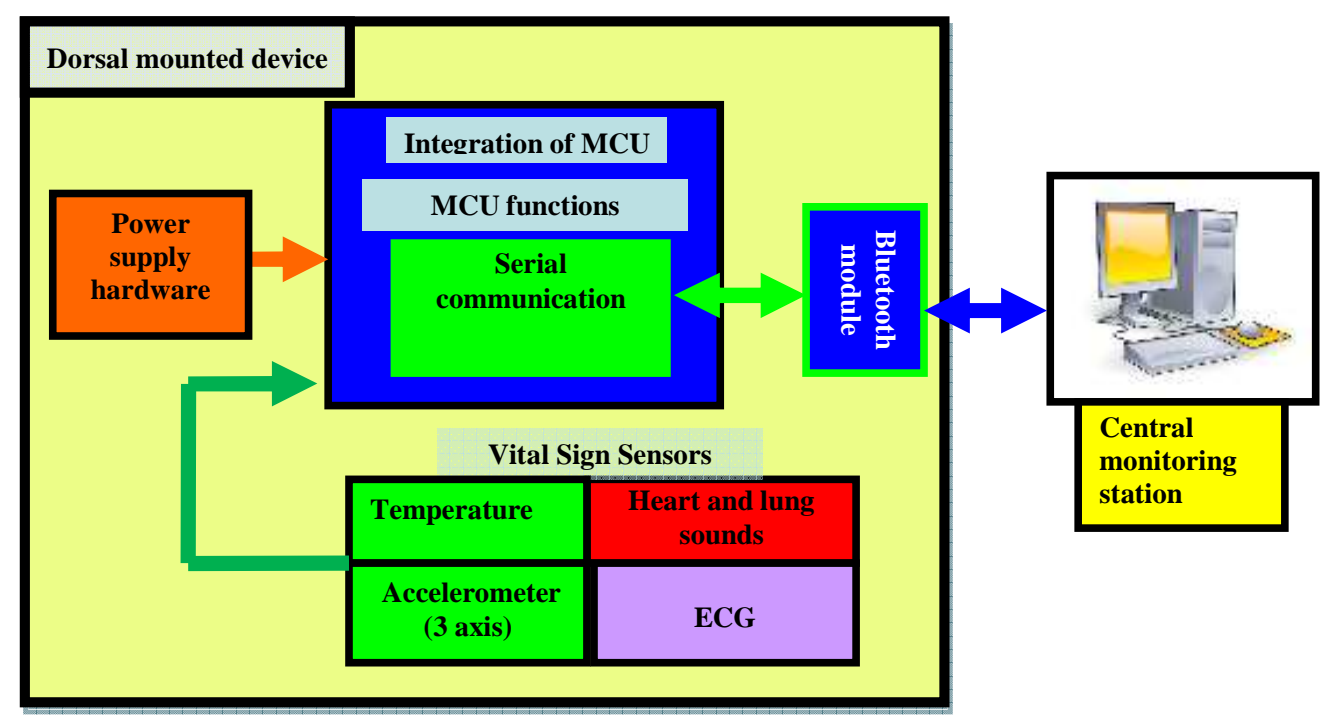

Figure 4-2 Block diagram of dorsal mounted system

\section{Microcontroller}

There are a number of microcontrollers commercially available with the required specifications for measuring ADC values and that can also transmit data using the universal asynchronous receiver/transmitter (UART) protocol. The selection used is based on previous experience with a certain microcontroller and the availability of development tools such as the appropriate compiler and programmer.

The Texas Instrument's MSP430FG439 microcontroller is a very low power microcontroller unit (MCU) developed for small modular low power monitoring equipment. The required specifications for the MCU are listed below: 
- Low supply voltage range: $1.8 \mathrm{~V}$ to $3.6 \mathrm{~V}$.

- Ultra low power consumption.

- At least six 12-Bit ADC.

- Serial communication interface (USART).

- $60 \mathrm{~kb}+256 \mathrm{~b}$ flash memory and $2 \mathrm{~kb}$ RAM.

The MSP430FG439 MCU fulfils all the above requirements and was selected for the prototype.

The analogue input pins are summarised in Table 4-2, and the digital inputs and outputs pins are summarized in Table 4-3.

Table 4-2 MCU analogue inputs

\begin{tabular}{|l|c|l|}
\hline Pin no. & Name & \\
\hline P6.5 & ACC_X & Voltage output of $x$-axis of accelerometer. \\
\hline P6.6 & ACC_Y & Voltage output of y-axis of accelerometer. \\
\hline P6.7 & ACC_Z & Voltage output of z-axis of accelerometer. \\
\hline P6.2 & TEMP & Voltage output of the DS600 analogue temperature sensor. \\
\hline P6.3 & ECG & Voltage output of the ECG monitoring device. \\
\hline P6.4 & SOUND & Voltage output of the lung sound transducer circuit. \\
\hline
\end{tabular}

Table 4-3 MCU digital inputs and outputs

\begin{tabular}{|c|c|c|}
\hline Pin no. & Name & Description \\
\hline P4.1 & ACC_EN & $\begin{array}{l}\text { Accelerometer enable - output high and the accelerometer is } \\
\text { on, output low and it is in sleep mode. }\end{array}$ \\
\hline P4.3 & ACC_GS2 & $\begin{array}{l}\text { Accelerometer range select } 2 \text {. For more information refer to } \\
\text { Section 4.2.2.4. }\end{array}$ \\
\hline P4.2 & ACC_GS1 & $\begin{array}{l}\text { Accelerometer range select } 1 \text {. For more information refer to } \\
\text { Section 4.2.2.4. }\end{array}$ \\
\hline P2.4 & MCU_TXD & $\begin{array}{l}\text { UART TX output that connects to the Bluetooth modules } \\
\text { UART RX input. For more information refer to Figure } 4-5 \text {. }\end{array}$ \\
\hline $\mathbf{P 2 . 5}$ & MCU_RXD & $\begin{array}{l}\text { UART RX input that connects to the Bluetooth modules } \\
\text { UART TX output. For more information refer to Figure 4-5. }\end{array}$ \\
\hline P4.0 & TEMP_on/off & Temperature chip on/off switch (not used in c-code). \\
\hline
\end{tabular}




\section{DEVELOPMENT OF DORSAL MOUNTED DEVICE}

\section{Bluetooth Module}

It is essential in this project to implement a wireless connection from the device worn by the patient to the central monitoring station. The standard output communication protocol for serial communication is UART which is converted to serial port profile (SPP) when connected to a Bluetooth stick in a personal computer (PC). The Bluetooth module should be able to transmit UART serial data at rates of at least $115200 \mathrm{bps}$ and be able to connect to a specific Bluetooth module every time the device is powered up. The original equipment manufacturer (OEM) Bluetooth module should be small, lightweight and be able to transmit data to at least 30 meters from the central monitoring station. The Parani ESD100 from Sena fulfils all of these requirements by being small $(27 \mathrm{~mm} \mathrm{~L} \mathrm{x} 30 \mathrm{~mm} \mathrm{~W}$ x $14 \mathrm{~mm} \mathrm{H})$ and weighing only 5 g. The range with the integrated on-board chip antenna is $100 \mathrm{~m}$ nominal. This is known as a class 1 Bluetooth module. Figure 4-3 shows the Parani ESD100 Bluetooth module that will be used to send data wirelessly [28].

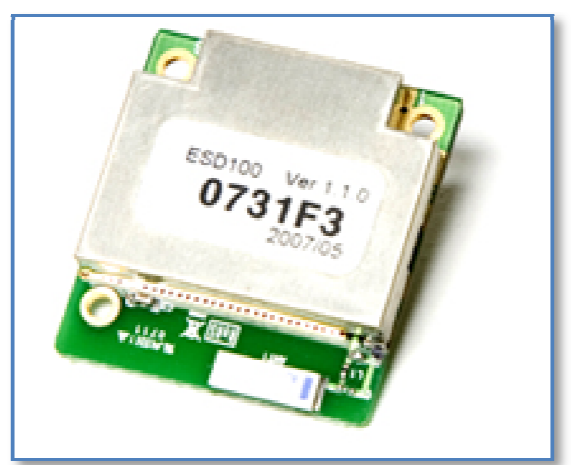

Figure 4-3 Parani ESD100 (SPP) Bluetooth module [28]

To implement two Bluetooth connections (one for the dorsal mounted device and one for the safety glove device) to one $\mathrm{PC}$ at the same time requires a simulation of a serial cable for the one Bluetooth connection and a normal Bluetooth to Bluetooth stick connection for the second connection. The dorsal mounted device will be connected to the serial port of the PC via the Bluetooth jig board simulating a serial cable with two Bluetooth modules. The $2^{\text {nd }}$ Bluetooth module (BM2) will plug into the jig board and will only connect to the Bluetooth module that is integrated in the dorsal mounted device (BM1). Figure 4-4 shows the jig board with the Bluetooth module that simulates a serial cable connection between the dorsal mounted device and the central monitoring station [29]. The Jig board converts the UART data received from the $\mathrm{BM} 2$ module to serial data that can be sampled using COM port 1 on the PC. 


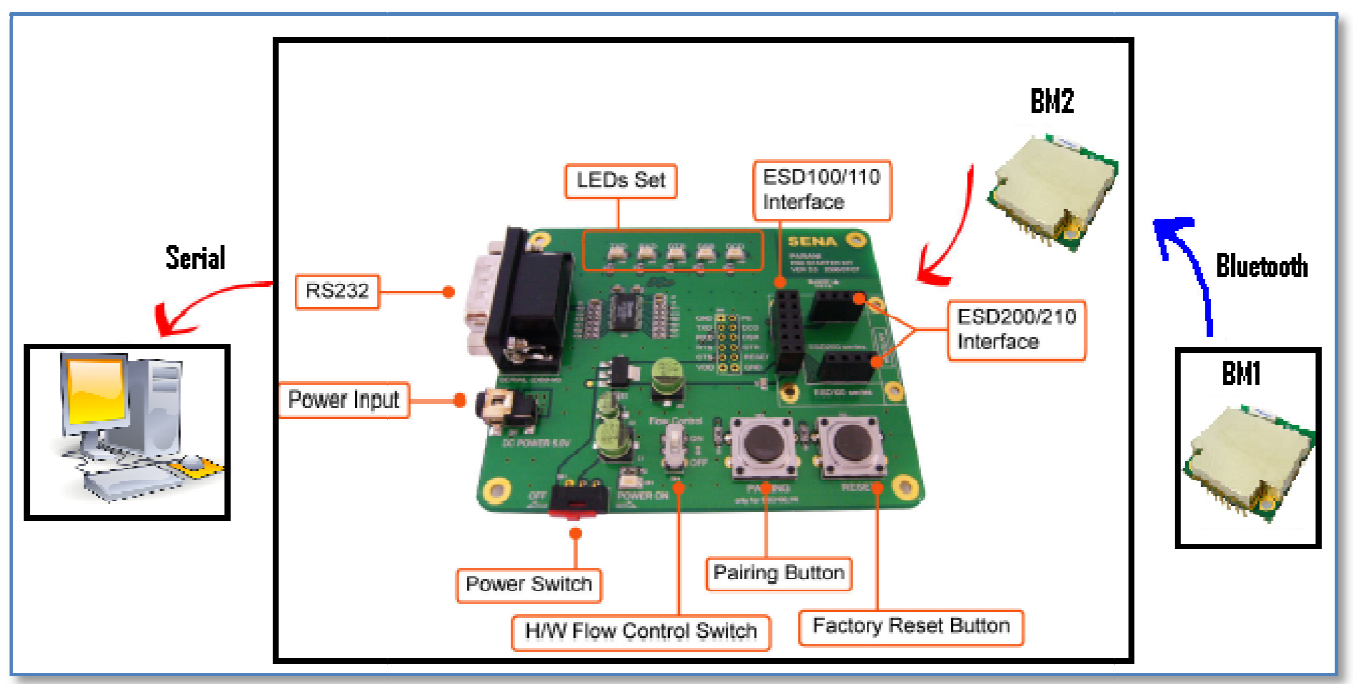

Figure 4-4 Jig board to simulate serial cable using two Bluetooth modules [29]

The ESD100 modules have to be programmed to be working at a selected baud rate and the device will connect to different Bluetooth modules. One of the four modes from Table 4-4 can be selected for each Bluetooth module [28]. The dorsal mounted device Bluetooth module connections are paired so that BM1 and BM2 can only connect to each other when power is supplied to these Bluetooth modules. This jig board is used to program the ESD100 modules to be paired.

Table 4-4 Mode description of Bluetooth module [28]

\begin{tabular}{|ll|}
\hline Mode & $\begin{array}{l}\text { Description } \\
\text { In this mode no response when power is on or when software is reset. This is } \\
\text { the default factory mode. }\end{array}$ \\
\hline Mode 1 & Parani -ESD tries to connect the last connected Bluetooth device. \\
\hline Mode 2 & Parani -ESD waits for a connection from the last connected Bluetooth device. \\
\hline Mode 3 & $\begin{array}{l}\text { Parani -ESD is waiting for the connection from any other Bluetooth devices. } \\
\text { In Mode } 3 \text { the Parani -ESD is discoverable and can be connected to by other } \\
\text { Bluetooth devices. }\end{array}$ \\
\hline
\end{tabular}

The wire connection of the Bluetooth module to the MCU is illustrated in Figure 4-5 [28]. The transmit pin of the Bluetooth module will connect to the MCU-Receive pin and the MCU-Transmit pin will connect to the receive pin of the Bluetooth module. The Bluetooth module's power supply should be regulated below $3.3 \mathrm{~V}$. The RST pin 
is connected to turn the Bluetooth module on and off from the MCU if the Bluetooth connection is not required to send any data for a period of time. This feature is not used due to the continuous data sending requirements (continuous monitoring of patient).

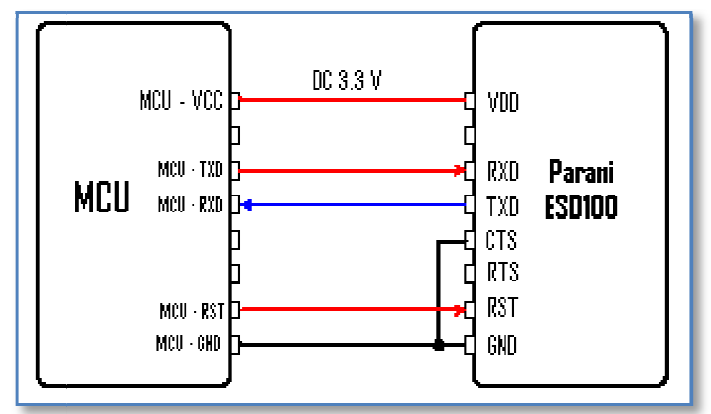

Figure 4-5 Connections of Bluetooth module to MCU [28]

\subsubsection{Hardware Realization of Sensors}

The hardware design of the sensors will cover each sensor in detail, beginning with a general indication of what should be measured with that sensor and then continue with a detail development of a circuit diagram. The measurement of ECG, sound (acoustic device), temperature and movement (accelerometer) will be explained.

\section{Electrocardiogram (ECG) Circuit}

The main component in the ECG circuit is Texas Instrument's INA322EA integrated circuit (IC), a micro power single-supply complementary metal-oxide-semiconductor (CMOS) instrumentation amplifier with a very favourable common mode rejection ratio (CMRR) of $60 \mathrm{~dB}$. The circuit also uses three operational amplifiers from a single CMOS quad op-amp package, together with small resistors and capacitors. These components are tiny surface-mounted parts in order to minimize noise and current draw. The design is based on a design provided in TI's datasheet for the INA322EA component and incorporates some of the design elements from a fourlead ECG system designed by researchers at MIT's Media Lab as part of the Every Sign of Life project [30]. 


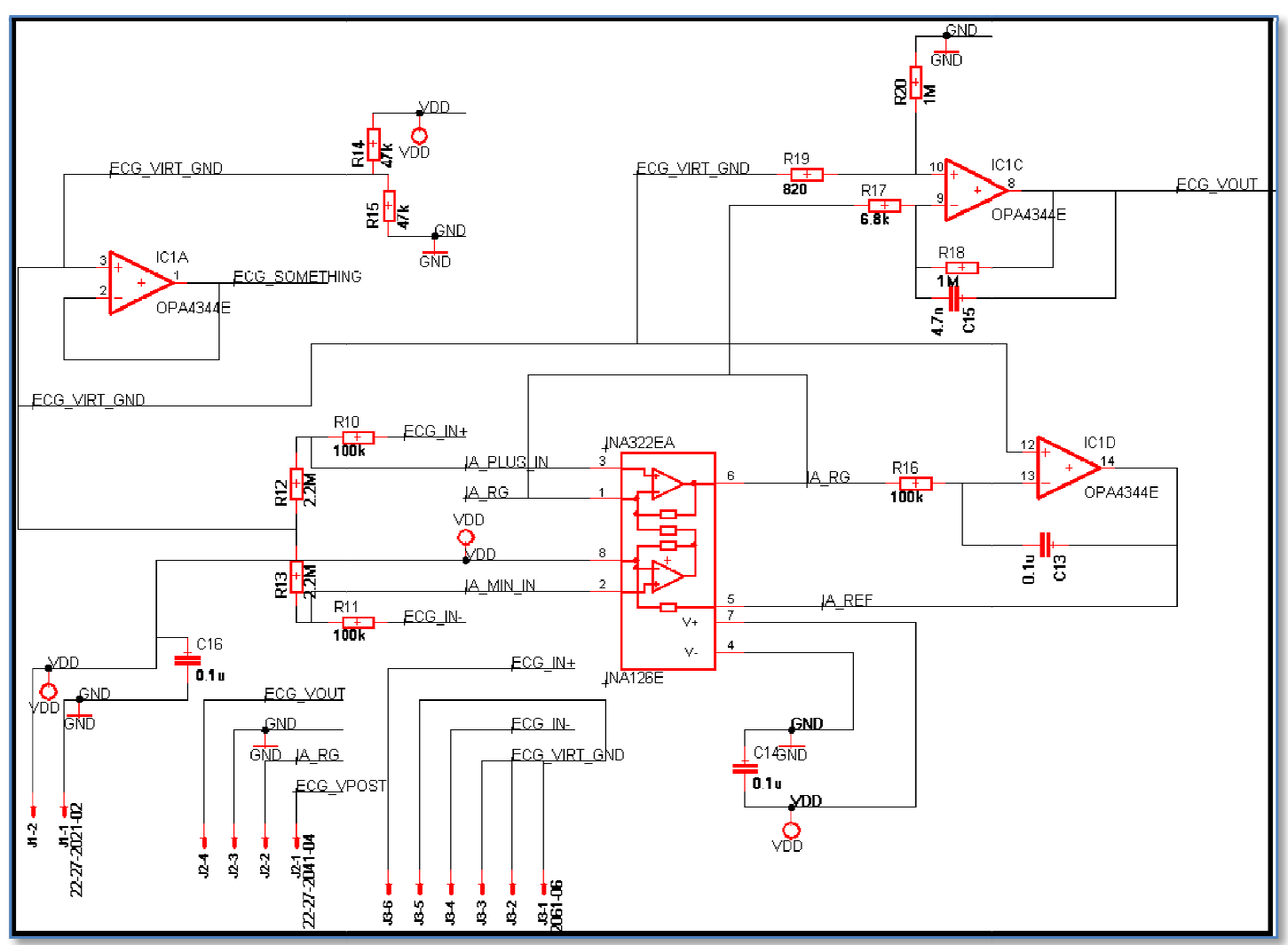

Figure 4-6 ECG amplifier circuit

The schematic figure (Figure 4-6) consists of a biasing divider (R14, R15) which splits the supply in half and connects to the lower left back lead which sets the patient to the correct potential. The other two leads receive signals from the patient's upper back and transmit the resulting differential signal to the INA322EA device. This component amplifies the difference by a factor of five (internally configured), while rejecting almost all of the common-mode noise. The INA322EA is configured with a highpass feedback filter to dynamically correct any DC shift that may occur over time. Its output passes to a final operational amplifier which further amplifies the signal and acts as a lowpass filter [31].

\section{Lung Sound Transducer Circuits}

The Panasonic WM-61A Omni directional Back Electrets Condenser Microphone Cartridge is used to sample the air chamber sound waves to electrical signals. A two stage operational amplifier circuit is used to amplify the electronic acoustic signals. The first stage inverting amplifier inverts the signal with amplification. 


$$
\operatorname{Gain}_{1}=\frac{\mathrm{V}_{1 \text { out }}}{\mathrm{V}_{1 \text { in }}}
$$

This first stage amplified output signal is then used as an input to the second stage amplifier which amplifies the signal another 10 times and inverts the signal again using equation 4.2, which leaves only the original signal amplified enough to detect respiratory sounds after the two stages of amplification [32].

$$
\operatorname{Gain}_{2}=\frac{V_{2 o u t}}{V_{2 \text { in }}}=-\frac{R 25}{\mathrm{R} 26}=-\frac{2.2 \mathrm{k}}{220}=-10
$$

An acoustic signal is generated around a ground reference and is alternating current (AC) amplified around this ground reference. To be able to use this acoustic signal in a single supply design using batteries, a virtual ground setup is used. The virtual ground is calculated using a voltage divider circuit as shown in Figure 4-7, with resistors R7 and R8. The voltage divider pair is connected to the positive pin of the operational amplifier circuit (IC2A). This circuit is configured as a buffer to ensure that the reference input voltage will be kept stable at $1.65 \mathrm{~V}$. The $1.65 \mathrm{~V}$ reference ground is connected to the amplification amplifier circuit's positive input pin (IC2B). This will ensure that $\mathrm{AC}$ amplification is made around the $1.65 \mathrm{~V}$ ground reference with rejection of DC amplification.

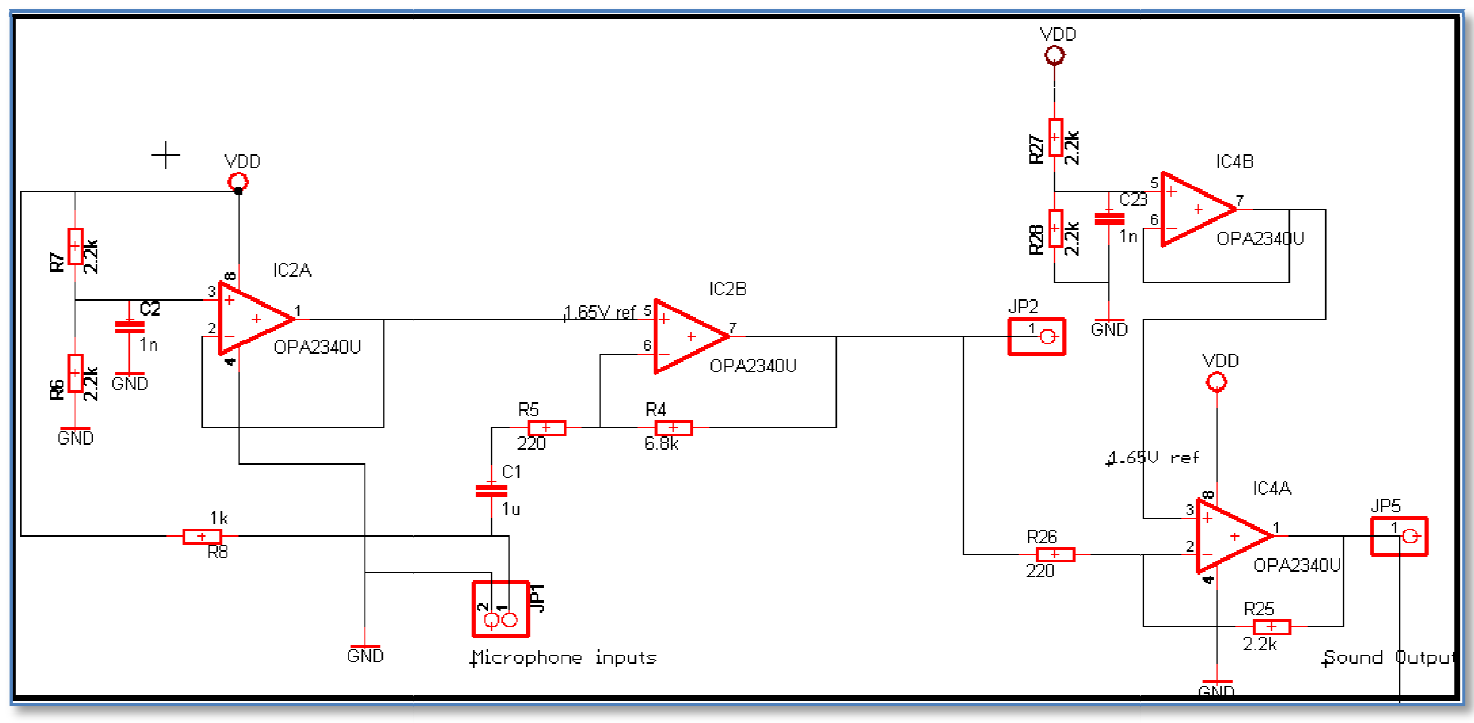

Figure 4-7 Lung sound transducer circuit 
III. Temperature Measurement Circuit

Progressive hypothermia can be prevented by early detection with a temperature probe. The Maxim DS600 analogue temperature sensor was selected due to its small size and good accuracy. The ADC on the MCU can sample at 12-bit resolution yielding a measurement accuracy of 4096 points. This chip is intended for portable medical equipment as mentioned in the datasheet [33]. This device has no external components, as can be seen from Figure 4-8, which helps to minimize the size on the PCB.

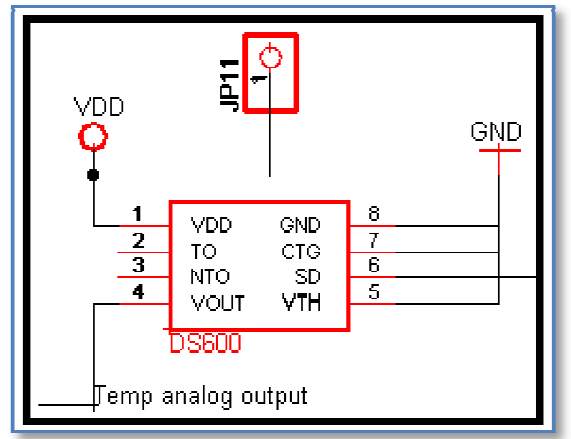

Figure 4-8 Analogue temperature circuit (DS600)

\section{Accelerometer Circuit}

The accelerometer is designed to measure the movement of the patient. When the patient is moving too much, the ECG and acoustic sound data will not be transmitted, to ensure that false readings are avoided as these sensors are sensitive to movement artefacts. When this occurs, a warning message will be displayed on the screen to ensure that the clinician should visit the patient to establish that the patient is safe from a medical emergency. The MMA7260QT is a 3-axis accelerometer manufactured by Freescale Semiconductor [34] and offers programmable dynamic ranges of $1.5,2,4$ and $6 \mathrm{~g}$ (where $1 \mathrm{~g}=9.81 \mathrm{~m} / \mathrm{s}^{2}$ ) and a controlled sleep mode. According to the datasheet the MMA7260QT makes use of internal switched capacitor filters to manage the frequency bandwidth [34]. These filters generate high frequency noise on the outputs and the use of an RC filter with $\mathrm{R}=1 \mathrm{k} \Omega$ and $\mathrm{C}=0.1$ $\mu \mathrm{F}$ is suggested. With reference to Figure 4-9, these requirements explain the values of $\mathrm{R} 2=\mathrm{R} 3=\mathrm{R} 4=1 \mathrm{k} \Omega$ and $\mathrm{C} 5=\mathrm{C} 6=\mathrm{C} 7=0.1 \mu \mathrm{F}$. The datasheet also suggests the use of a ceramic power supply noise decoupling capacitor $\mathrm{C} 8=0.1 \mu \mathrm{F}$ [34]. 


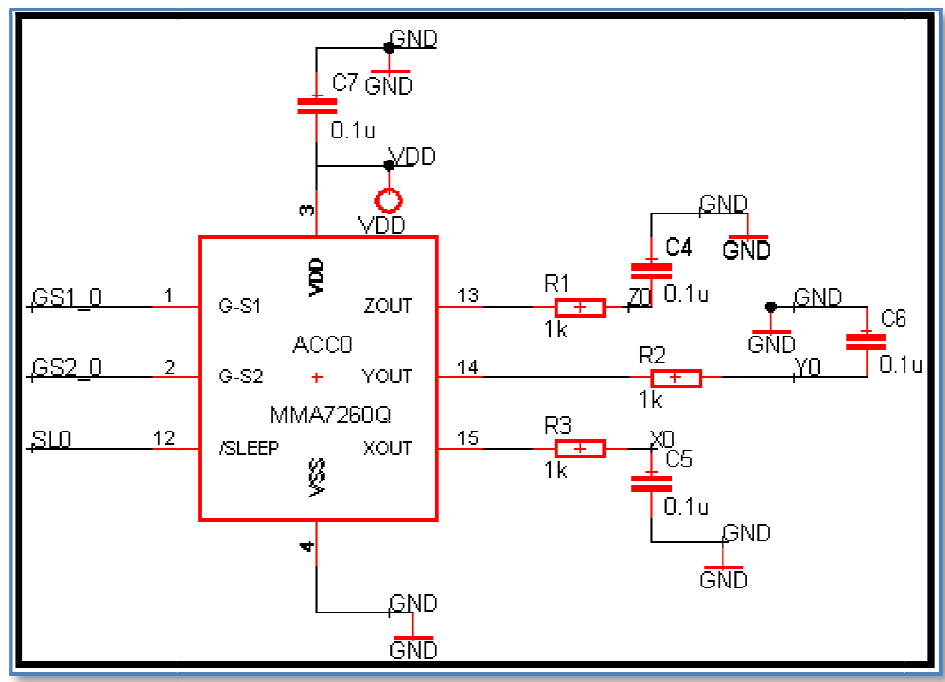

Figure 4-9 Accelerometer (MMA7260QT) circuit [34]

ACC_EN, ACC_GS1 and ACC_GS2 are the digital control pins that connect to digital outputs of the MCU (P4.1, P4.2 and P4.3 respectively). The accelerometer will only operate with an active high on ACC_EN and be in sleep mode with an active low. ACC_GS1 (g-Select1) and ACC_GS2 (g-Select2) control the sensitivity of the accelerometer and this is summarised in Table 4-5. The three analogue outputs ACC$\mathrm{X}, \mathrm{ACC}-\mathrm{Y}$ and ACC-Z will connect to the ADC input pins of the MCU (P6.5, P6.6 and P6.7 respectively).

Table 4-5 Accelerometer sensitivity selection

\begin{tabular}{|c|c|c|c|}
\hline g-Select 2 & g-Select 1 & g-Range & Sensitivity \\
\hline 0 & 0 & $1.5 \mathrm{~g}$ & $800 \mathrm{mV} / \mathrm{g}$ \\
\hline 0 & 1 & $2 \mathrm{~g}$ & $600 \mathrm{mV} / \mathrm{g}$ \\
\hline 1 & 0 & $4 \mathrm{~g}$ & $300 \mathrm{mV} / \mathrm{g}$ \\
\hline 1 & 1 & $6 \mathrm{~g}$ & $200 \mathrm{mV} / \mathrm{g}$ \\
\hline
\end{tabular}

\subsection{Microcontroller Software Design}

In this section the design of the MCU C-code is presented. All the different calculations and setup procedures will be explained. The use of timers to calculate the sampling rate of the ADC converter will also be discussed in detail. To sample the six analogue sensors, six 12-bit ADC channels are used. 


\subsubsection{Program Flow Diagram}

The MCU C-code program flow diagram is shown in Figure 4-10. When the MCU is powered up, its start-up sequence is initiated. In the start-up sequence the UART, $\mathrm{ADC}$ and other input and output pins are set up. After the start-up sequence the MCU initialises into a continuous while loop and waits for an interrupt. The ADC interrupt will happen at a rate of $1600 \mathrm{~Hz}$ as setup using Timer A. Inside the interrupt the sampling of ADC channels and sending of data packets will be handled as shown in the program flow diagram of Figure 4-10. When the one data packet is sent, the interrupt will return to the main while loop and wait for the next interrupt to occur. This process will continue until the device is switched off.

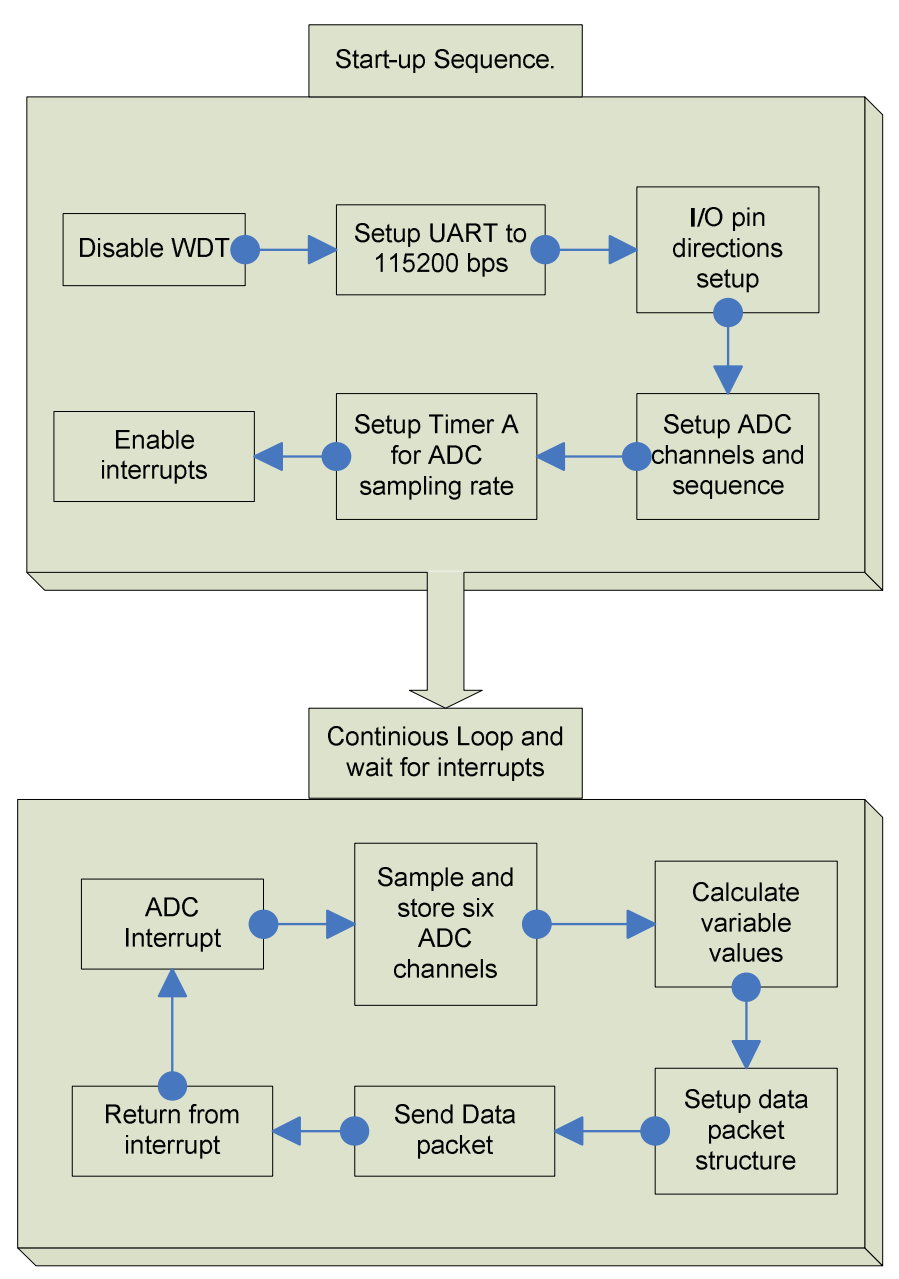

Figure 4-10 MCU program flow diagram 


\subsubsection{ADC}

The six ADC channels are set up to sample at $1600 \mathrm{~Hz}$. All the ADC channels will have the same sampling rates. A single timer is used to calculate this rate and only one register can be changed for this calculation. The ADC sampling rate is set up using the Timer A of the MCU (to be discussed in Section 4.3.4). The repeated sequence channels sampling mode was chosen from the datasheet of the MCU, as illustrated in Figure 4-11 below.

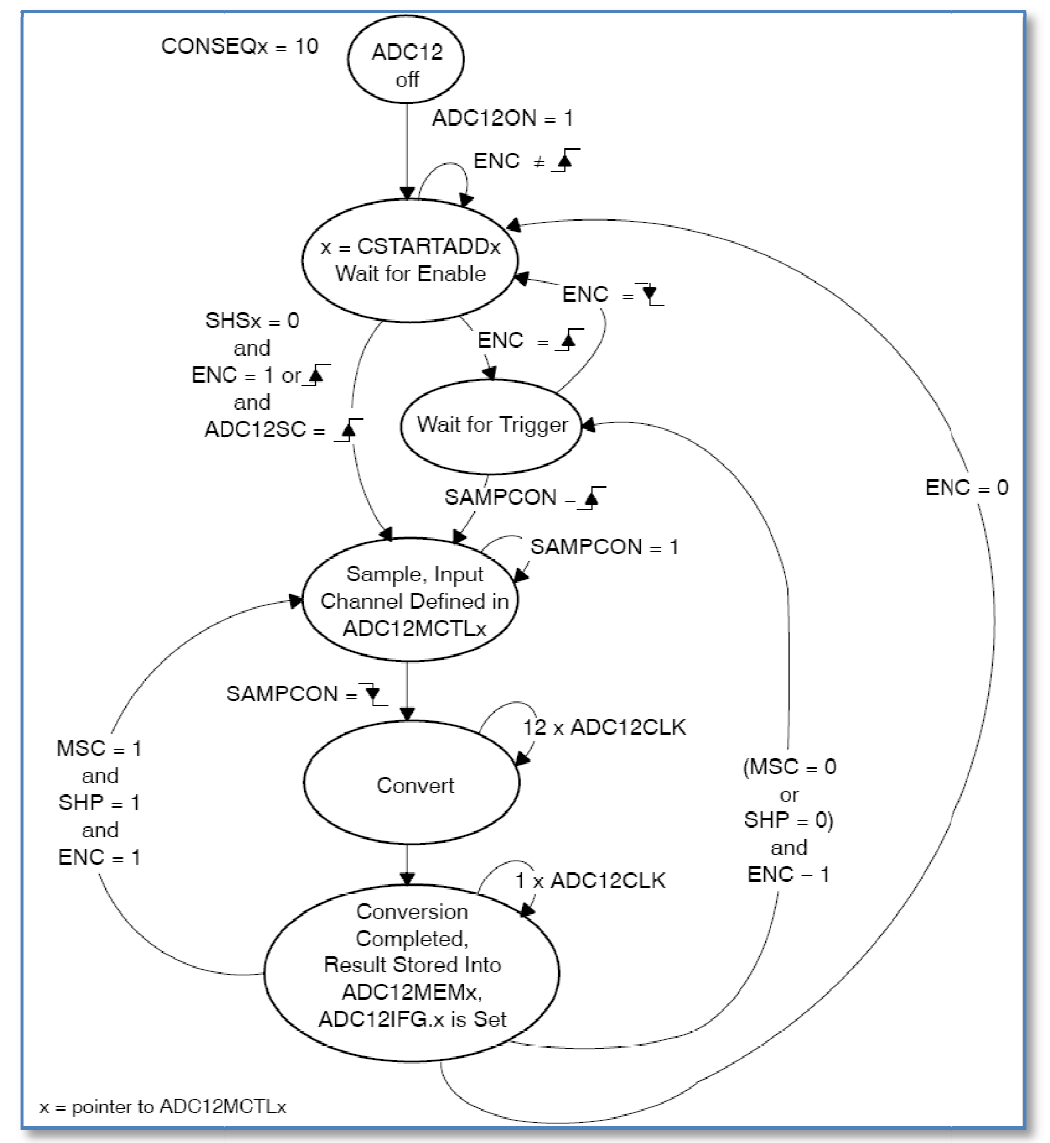

Figure 4-11 Repeated sequence of channels ADC sampling [35]

This mode will sample all the channels that you select in a sequence until the end of the sequence. An end of sequence (EOS) register is set for detection of the EOS. At the EOS the first channel will be sampled again and the sequence will continue until the ADC sampling is set off. The ADC12 sampling will call an interrupt at each EOS and store the sampled ADC channels in six different variables. These six variables can then be changed and prepared for sending using the UART protocol that will be discussed below. 


\subsubsection{UART and Communication Protocol}

The baud rate for serial communication is set up from the clock frequency used to run the MCU. The MCU is using an $8 \mathrm{MHz}$ external clock. This value is used to calculate the desired register values that should be set for an 115200 baud rate that is required for communication with the ESD100 Bluetooth device. The calculation was done using a dedicated website for calculation of the baud rate for this specific device [36]. The calculation indicated that for this baud rate at the selected frequency the UBR00 register should be set to 0x45, the UBR10 should be 0x00, and the UMCTL0 register should be 0x29. This was done and successful serial (Bluetooth) communication was tested with these values set at a baud rate of $115200 \mathrm{bps}$.

\section{Sending Serial Package Data}

For sending five channels of data over one serial connection an identifier is needed to identify the beginning of a specific serial data package. The first byte of the data package indicated in Figure 4-12 is a 1 byte identifier (0xFF hexadecimal value) following with five bytes for the data channels. The $2^{\text {nd }}$ byte is the 8 -bit analogue temperature value. The $3^{\text {rd }}$ byte is an 8 -bit ECG value (the 12-bit value is divided by 16 to scale it to an 8-bit value).

\begin{tabular}{|c|c|c|c|c|c|}
\hline 1 Byte & 1 Byte & 1 Byte & 1 Byte & 1 Byte & 1 Byte \\
\hline StartByte (0xFF) & Temperature & ECG & Sound_H & Sound_L & Acc_average \\
\hline
\end{tabular}

Figure 4-12 Serial data package

Serial data can only send 8-bit values, to be able to send 12-bit values directly the 12bit value should be divided into two 8-bit values: a high byte and a low byte. The sound recording is a 12-bit ADC value and is divided into these two 8-bit values as shown below:

$$
\begin{aligned}
& \text { Sound }_{L}=(\text { Sound \& 127) } \\
& \text { Sound }_{H}=\frac{(\text { Sound \& 3968) }}{128}
\end{aligned}
$$

For example, a 12-bit value of 2187 decimal (00100010001011 binary) will be divided for the low byte into 11 decimal (0001011 binary) and for the high byte into 17 (0010001 binary) using equation 4.3 and 4.4 respectively, when combining the high and low byte, and removing the $7^{\text {th }}$ bit from the low byte and high byte, the original binary value of 00100010001011 is revealed. The $5^{\text {th }}$ byte is calculated using the average of the three sampled $\mathrm{ADC}$ values for the accelerometer $\left(\mathrm{Acc}_{\mathrm{Avg}}\right)$, as shown in equation 4.5 . This 12-bit value is then recalculated to an 8-bit value by dividing the 12-bit value by 16 . 


$$
A c c_{A v g}=\frac{\left(A c c_{x}+A c c_{y}+A c c_{z}\right)}{3}
$$

This serial data package is sent constantly over the Bluetooth connection. Figure 4-13 shows an example of two sequential data packages sent.

\begin{tabular}{|c|c|c|c|c|c|c|c|c|c|c|c|}
\hline 1 Byte & 1 Byte & 1 Byte & 1 Byte & 1 Byte & 1 Byte & 1 Byte & 1 Byte & 1 Byte & 1 Byte & 1 Byte & 1 Byte \\
\hline SB & Temp & ECG & S_H & S_L & Acc & SB & Temp & ECG & S_H & S_L & Acc \\
\hline
\end{tabular}

Figure 4-13 Example of serial data format

\subsubsection{Timer A Setup}

Timer A is used to set the desired $1600 \mathrm{~Hz}$ sampling frequency. Timer $\mathrm{A}$ is set up to use the $8 \mathrm{MHz}$ auxiliary clock (ACLK). This value is used when calculating the values for the timer registers. Timer A is also set up in "up" mode: in this mode the timer will count until it reaches the maximum selected value and is then reset. This will ensure that the timer will always count a fixed length of time. The maximum selected value is selected by changing the Timer A register to a value determined by equation 4.6.

$$
\operatorname{TACCRO}=\frac{\left(\frac{8000000}{1600}\right)}{6}=833
$$

The $8 \mathrm{MHz}$ value is divided by the desired frequency value of $1600 \mathrm{~Hz}$. This value is then divided by the six sampling channels.

\subsection{Mechanical Design of Dorsally Mounted Enclosure}

In this section, all the design considerations will be explained that influenced the mechanical design of the dorsally mounted enclosure. After this a detail description of the CAD model will be given with the use of drawings and pictures of the manufactured prototype.

\subsubsection{Design Considerations for Each Sensor}

All the sensors have their own design requirements. All of these requirements should be considered when developing a single unit that accommodates these sensors. Firstly the protection enclosure should be large enough to fit the PCB with the Bluetooth module, the battery supply unit, the air-coupled microphone unit and all the measurement electrodes. 


\section{ECG Electrodes}

There are three ECG electrodes from the 2-lead ECG monitor that should be fitted inside the device. ECG electrodes are normally placed on the chest of the patient, as shown in Figure 4-14 to obtain the basic ECG (PQRST) rhythm strip.

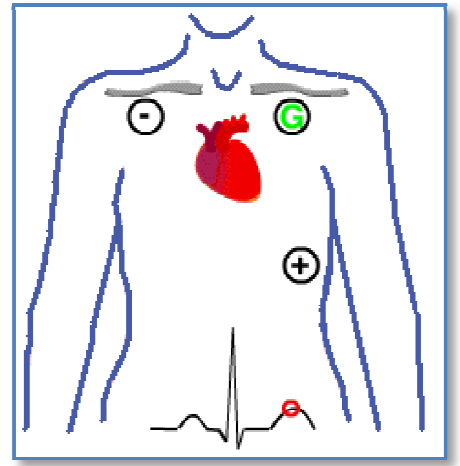

Figure 4-14 Chest ECG placement of probes

With this device the positioning of the ECG electrodes is on the back of the patient. To measure the same ECG rhythm as if on the front of a patient, the placements appear reversed to obtain the same electrical vector across the heart. The placement used in the proposed device is shown in Figure 4-15.

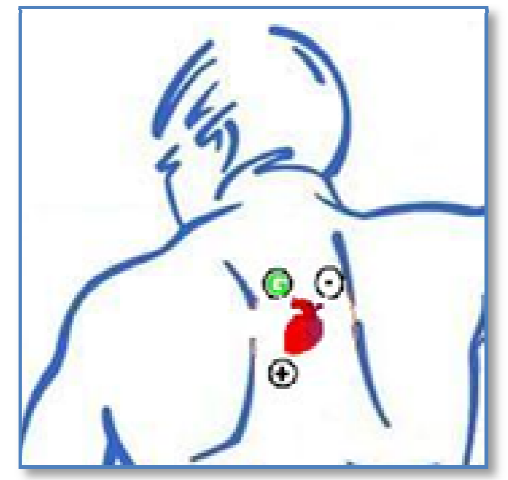

Figure 4-15 Dorsal ECG placement of probes

The placement of the ECG electrodes limits the size of the device as the electrodes should be placed underneath the device to ensure that the patient cannot access the electrodes or wires. The distance between the positive and negative leads is proportional to the amplitude of the measured ECG waveform. The further they are from each other, the larger the signal will be. The length between these two 
electrodes should be large enough to ensure that the signal to noise ratio (SNR) of the small ECG waveform measured on the back of the patient is high enough to recognize the PQRST rhythm. Digital filters are used to filter out most of the unnecessary noise caused by movement artefacts of the electrodes and electrical noise.

No usable results were obtained from the "Ergonomic design: anthropometry and environment" paper [37] and other research on the distance between the right and left scapula, therefore the distances between the left and right scapula for volunteers were measured as shown in Table 4-6.

Five volunteers' sizes were measured between their left and right scapulas to calculate the distance between the top two electrodes when the volunteers were sitting in a normal upright position and with the volunteers pushing back their shoulder, which moves the left and right scapulas closer to each other. The top two ECG electrodes should be between these two scapulas to measure the best electrical signal.

Table 4-6 Distance between left and right scapulas

\begin{tabular}{|l|c|c|}
\hline $\begin{array}{c}\text { Volunteers } \\
\text { (size) }\end{array}$ & $\begin{array}{c}\text { Normal sitting position } \\
(\mathbf{m m})\end{array}$ & $\begin{array}{c}\text { Shoulders pushed back position } \\
(\mathbf{m m})\end{array}$ \\
\hline 1 (Large) & 180 & 100 \\
\hline 2(Small) & 150 & 100 \\
\hline 3(Large) & 160 & 110 \\
\hline 4(Medium) & 170 & 120 \\
\hline 5(Medium) & 160 & 100 \\
\hline
\end{tabular}

From Table 4-6 the minimum distance between the left and right scapulas for the volunteers when pushing back the shoulders was $100 \mathrm{~mm}$. Therefore the distance between the top two electrodes is designed to be $70 \mathrm{~mm}$, as shown in Figure 4-16. With this distance the top electrodes will be placed to the left and right of the vertebral column.

To ensure that the device is as small as possible, the length of the device was calculated using the length of the PCB and battery and the top and bottom ECG electrodes. The size of the PCB including the Bluetooth module is $70 \mathrm{~mm}(\mathrm{~L}) \times 32$ $\mathrm{mm}(\mathrm{W})$ and the size of the batteries is $50 \mathrm{~mm}(\mathrm{~L}) \times 32 \mathrm{~mm}(\mathrm{~W})$. To keep the device flat so that the patient can lie on his back while wearing the device, the batteries are placed next to the PCB towards the bottom of the device, as shown in Figure 4-16. 
The maximum length between the positive ECG electrode (bottom) and the negative ECG electrode (top) is $125 \mathrm{~mm}$ and is calculated by using the total length of the PCB and batteries of $120 \mathrm{~mm}$. The $125 \mathrm{~mm}$ used in the design is large enough to measure a good quality ECG waveform, as will be shown in Chapter 6.

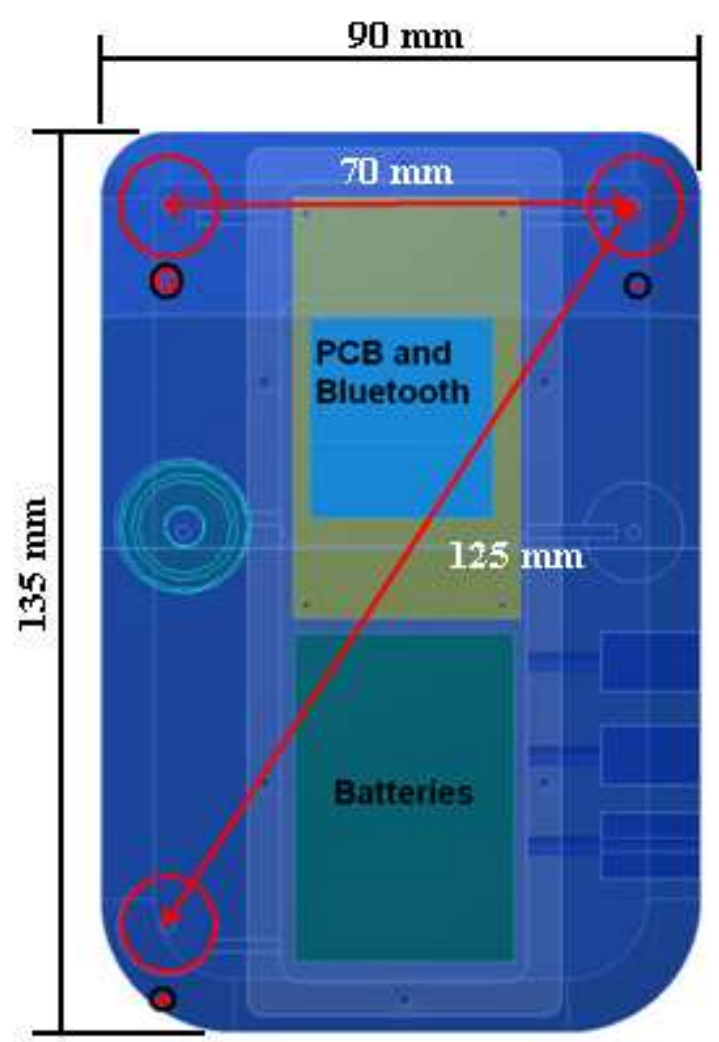

Figure 4-16 Placement of ECG electrode, batteries and PCB

Now that the position of the ECG electrodes has been determined, the design of the measurement probes should be considered. Normal commercial ECG electrodes are used and connected to the device each time the device is attached to a new patient, using clips that connect to the commercial ECG electrodes [38]. The clips are attached to the device using a spring-loaded system to minimise the movement of the probes on the skin, relative to movement of the device on the back of the patient. Figure 4-17 shows a typical commercial ECG probe that is connected to the clip and spring-loaded system. The spring ensures that the clip can translate and rotate, giving the ECG electrode a lot of independent movement from the device. 


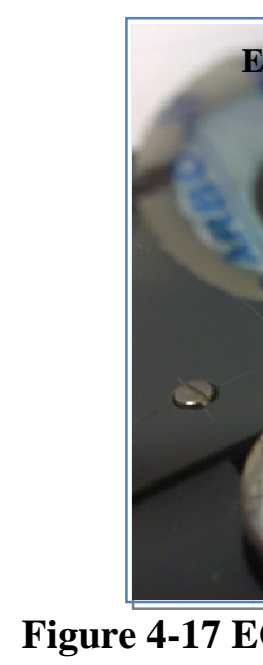

II. Air-Coupled N

To measure respiratory of the patient should bs minimum. To ensure the a unit that amplifies the

An air-coupled microp microphone element is ] skin using a rubber o-ri the chamber cavity, whe they are converted to ele

The rubber band used to o-ring which is placed superglue. A mechanica according to the above $\mathrm{s}$

Figure 4- 
The small circle in the middle of Figure 4-18 is where the Panasonic WM-61A condenser microphone should be press-fitted. The internal conical shape is designed for the air-coupled chamber. Its diameter should be between $10 \mathrm{~mm}$ and $15 \mathrm{~mm}$ and the depth of the cone between $2 \mathrm{~mm}$ and $5 \mathrm{~mm}$ to give maximum air pressure fluctuations inside the air chamber. For this design the conical shape specifications were selected to be $14 \mathrm{~mm}$ in diameter and $4 \mathrm{~mm}$ in depth, and resulted in significant air pressure fluctuations, and therefore quality measurements of heart and respiratory sounds are obtained.

The dorsal mounted device has a hole inserted $11 \mathrm{~mm}$ deep to accommodate the movement of the air-coupled microphone unit. The air-coupled microphone unit is also fitted on a spring system to ensure that the unit will always be pressed tightly against the skin of the patient to ensure that the air chamber is sealed during measurements, even when the dorsal mounted device moves away from the skin.

\section{Temperature Probe}

The analogue DS600 temperature module is situated inside the protection enclosure on the PCB. The contact pin of the sensor is extended using a short wire to an ECG electrode adhered to the skin of the patient. The ECG electrode is also fitted to a clip and spring-loaded system as discussed above.

\subsubsection{CAD Prototype Design of Electronic Protection Enclosure}

The size of the PCB had to be kept to a minimum. All the electronic components were fitted close to each other to ensure that a very small PCB is obtained. The size of the PCB is $63 \mathrm{~mm}(\mathrm{~L})$ by $35 \mathrm{~mm}(\mathrm{~W})$. The two-cell battery supply of $7.2 \mathrm{~V}$ should be regulated to $3.3 \mathrm{~V}$ to ensure that the microcontroller and the Bluetooth modules maximum input voltage never exceed $3.3 \mathrm{~V}$. The regulation is done using the TLV1117-33 3.3 V voltage regulator from Texas Instruments [40]. This regulator needs a minimum input supply voltage of $4.7 \mathrm{~V}$, which makes it a low-dropout voltage regulator. This power regulator circuit together with the electronics implemented in the initial prototype and Bluetooth module circuit should fit onto this PCB. Eagle version 4.16r1 from Cadsoft was used to design the PCB [41]. For PCB board layout for manufacturing, refer to Appendix B1.

CAD was used to design a computer model with all the design considerations discussed above. All the external components (battery, PCB and Bluetooth module) were drawn on scale and tested so that they fit inside the protection enclosure without interfering with the external protection enclosure. The batteries, PCB and Bluetooth module fit onto a small $2 \mathrm{~mm}$ thick base plate. The base plate with the components fit inside the bottom of the device, ensuring that only the charging points and the on/off switch are visible after the device is pressed against the patient's back, as shown in 
Figure 4-19. The whole dorsal mounted device is chamfered so that there are no sharp corners. This was done to ensure that the patient cannot injure anyone with the device. The outer dimensions of the dorsal mounted device are $90 \mathrm{~mm}(\mathrm{~W}) \times 135 \mathrm{~mm}$ (L) x $20 \mathrm{~mm}(\mathrm{H})$. For detail design sketches with manufacturing dimensions refer to Appendix C1.

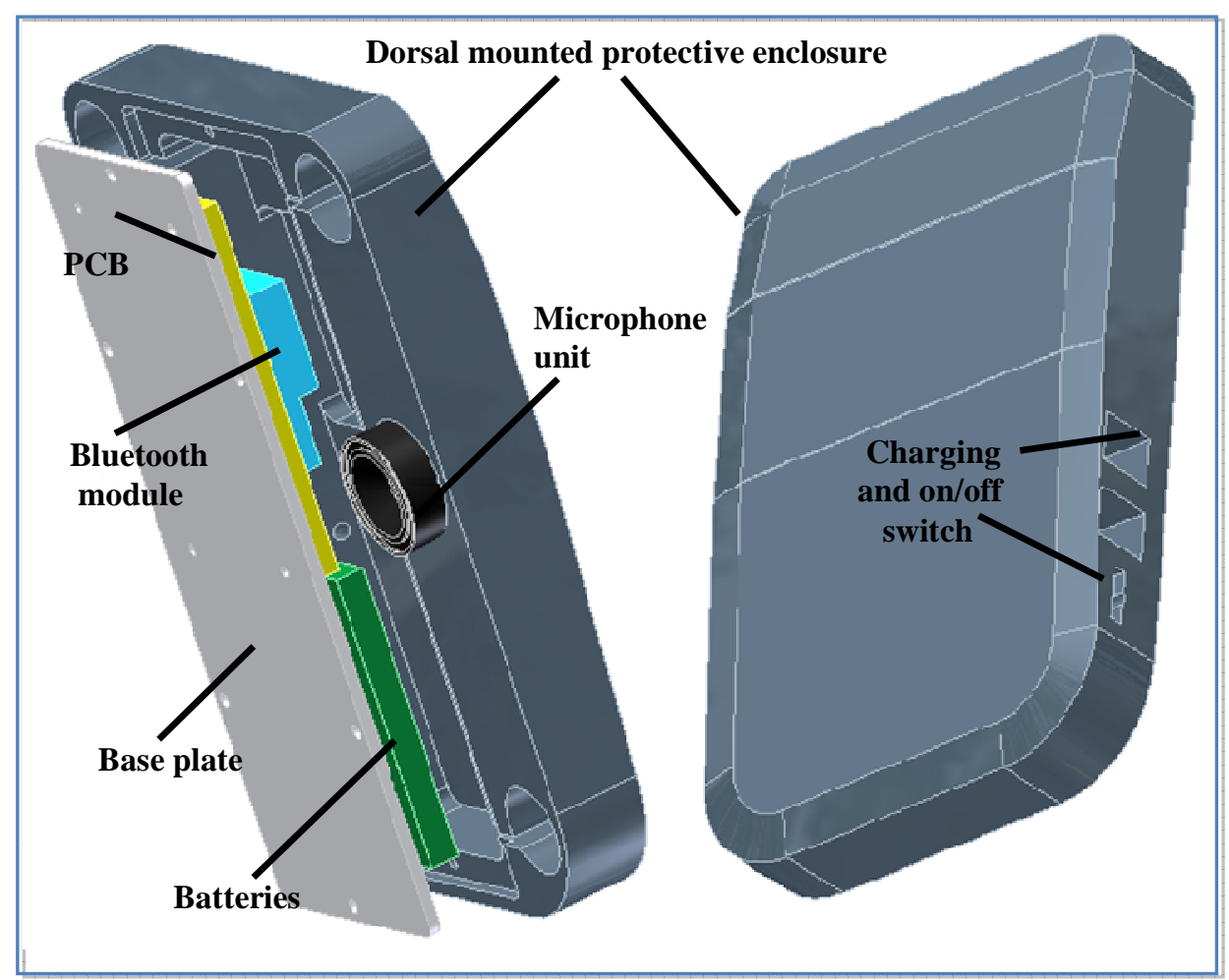

Figure 4-19 CAD model including batteries, PCB and Bluetooth module

\subsubsection{Manufacturing of the Prototype Protection Enclosure}

The dorsal mounted device, thin base plate and sound unit were manufactured using hard grey polymer of vinyl chloride plastic. This material was selected due to its strength and ease of machine-ability.

\subsection{Finalizing the Prototype}

\subsubsection{Combining Electronics and Mechanical Prototype}

The connection of the wires is done with the PCB and batteries mounted to the base plate shown in Figure 4-20. When all the sensors and charging wires are connected, the device can be closed using screws. When the base plate is closed, no wires are visible or accessible. 


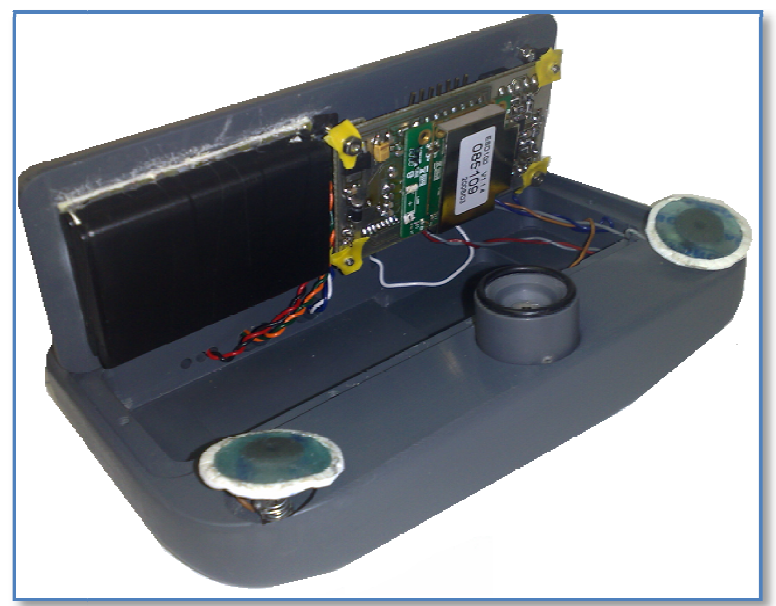

Figure 4-20 Fitting electronics inside dorsal mounted device

\subsubsection{Proposed Fitting of Prototype to the Patient}

The dorsal mounted device will be placed on the back of the patient between the left and right scapula. The ECG electrodes will be placed to the right and left of the patient's vertebral column. To ensure that the device stays in place and reduce the movement of the electrodes on the skin a very strong adhesive plaster will cover the device and keep the device attached to the skin between the left and right scapula. Figure 4-21 shows the device attached to a volunteer using an opsite flexigrid adhesive plaster $(150 \mathrm{~mm} \times 200 \mathrm{~mm})$.

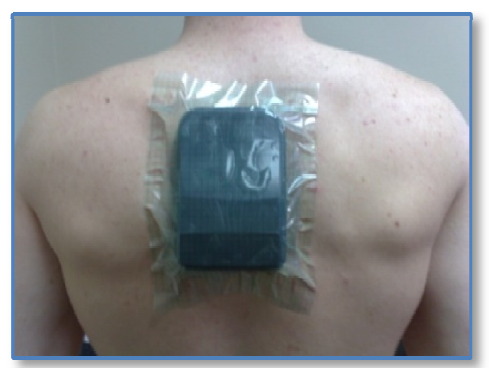

Figure 4-21 Adhesive plaster attachment of device on back of a volunteer

\subsection{Chapter Summary}

In this chapter the design of the dorsally mounted device was presented. A detail design of all the relevant electronics was given, followed by a detail design of the housing of the sensors, PCB and batteries. A final prototype was developed and shown. The proposed positioning of the device to a patient was shown. 


\section{CHAPTER 5}

\section{DEVELOPMENT OF THE SAFETY GLOVE DEVICE}

This chapter will discuss the design of the safety glove. Firstly, the initial prototype will be discussed with all the relevant electronic components, and then a design of the PCB including a Bluetooth connection will be explained. This design will include a detail development of the pulse oximeter and a detail design of the housing for the electronics. The protection of the device by means of a glove will also be discussed.

\subsection{Safety Glove Device Specifications}

The proposed device will be mounted on the wrist with the finger clip probe fitted to the index finger. The protecting glove will be attached over the hardware enclosure and finger clip to protect the wires and the probe. This device will measure the two PPGs and calculate the oxygen saturation ratio value from the two PPG signals (red and infrared light). To ensure that the device is as small as possible, one microcontroller is used to sample the red and infrared channels and send the red PPG signal together with the calculated ratio value using a serial communication protocol to the host system using a dedicated Bluetooth connection. Figure 5-1 shows a picture of the final safety glove prototype without the protective glove.

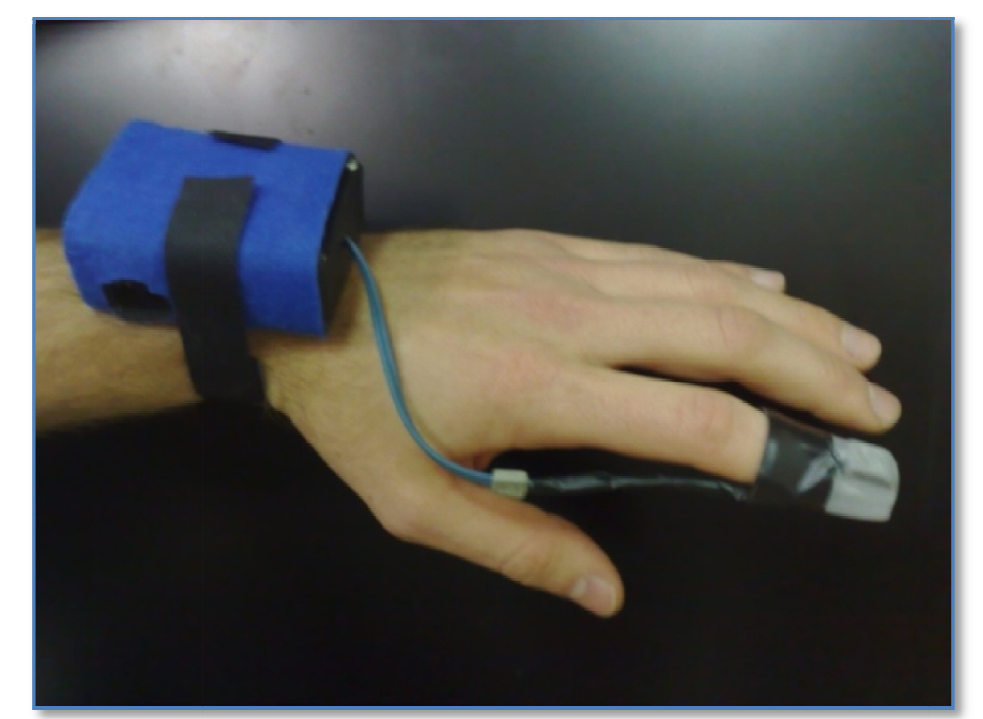

Figure 5-1 Final prototype without the protective glove 


\subsection{Electronic Design}

To determine the oxygen saturation, a microcontroller with ADC inputs and DAC outputs is used. This circuit drives the two LEDs needed to measure oxygen saturation. To send the calculated ratio value wirelessly to the central monitoring station, a dedicated serial port profile Bluetooth connection is established using a Parani ESD100 Bluetooth module from Sena and a USB Bluetooth stick.

\subsubsection{Selection of Main Electronic Components}

Upon inspection of the block diagram in Figure 5-2, the selection of the main components needed is clear. A microcontroller is required for sending the calculated data and for sampling of PPG data from the sensors, as well as for the management of the light intensity of the LEDs. A Bluetooth module is also needed as well as a battery supply unit. The selection of the specific components is discussed below.

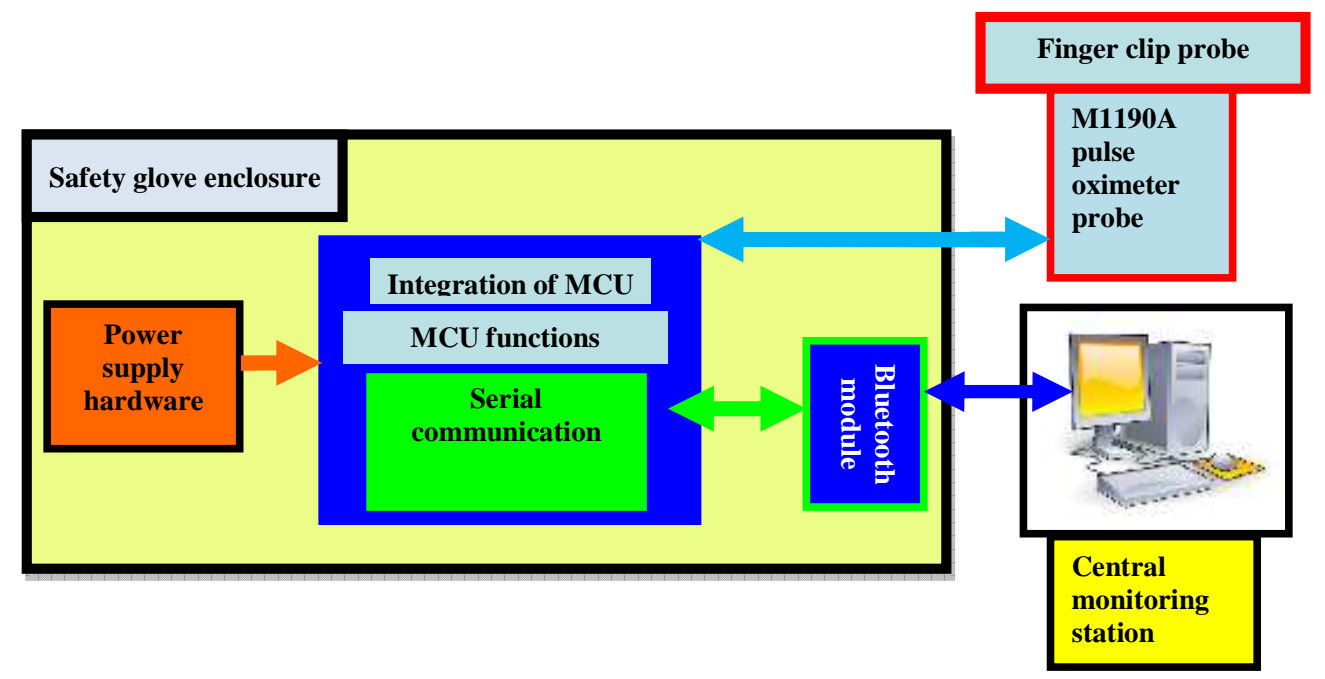

Figure 5-2 Block diagram of safety glove system

I. Microcontroller

There are many different microcontrollers on the market with the appropriate specifications for the implementation of a pulse oximeter. Texas Instruments has a specific medical section covering the development of ECG or pulse oximeter using a single chip design [42]. The microcontroller suggested by Texas Instruments for this application is a MSP430FG439 MCU. A document covering the implementation of the pulse oximeter using this microcontroller is available on the Texas Instruments website [43]. This document was used as a guide for development of this prototype. 
The MSP430FG439 microcontroller is a very low power MCU developed for small, modular, low power monitoring equipment. The MSP430FG439 MCU meets all the requirements for the prototype and was selected for the design of the pulse oximeter. The development board in Figure 5-3 was used to implement the initial prototype of the pulse oximeter design.

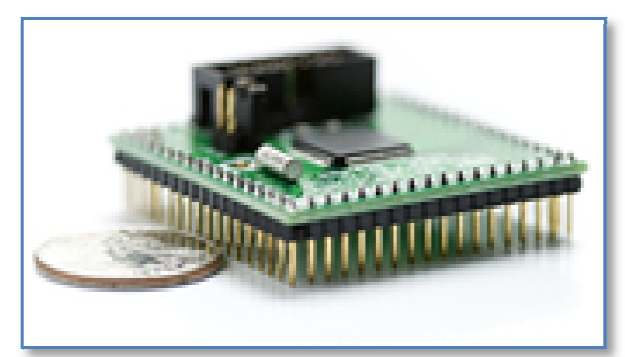

Figure 5-3 Low power MPS430FG439 development board

\section{Bluetooth Module}

As discussed in Section 4.2.1, the Parani ESD100 Bluetooth module will also be used for the safety glove device. This device will be connected using a normal serial port profile interface Bluetooth connection. The Parani ESD100 module is programmed in mode 1 (see Table 4-4) to connect to the previously connected Bluetooth stick every time the module is powered up. This connection will implement a virtual COM port. The GUI is set up to sample the safety glove data from this specific virtual COM port, at a baud rate of $115200 \mathrm{bps}$.

\subsubsection{Hardware Realization}

Figure 5-4 depicts a block diagram of the single chip pulse oximeter design. The figure shows all the small components situated inside the MCU that needs to be programmed and implemented to calculate the ratio value $(\mathrm{R})$ for the calculation of oxygen saturation [44]. 


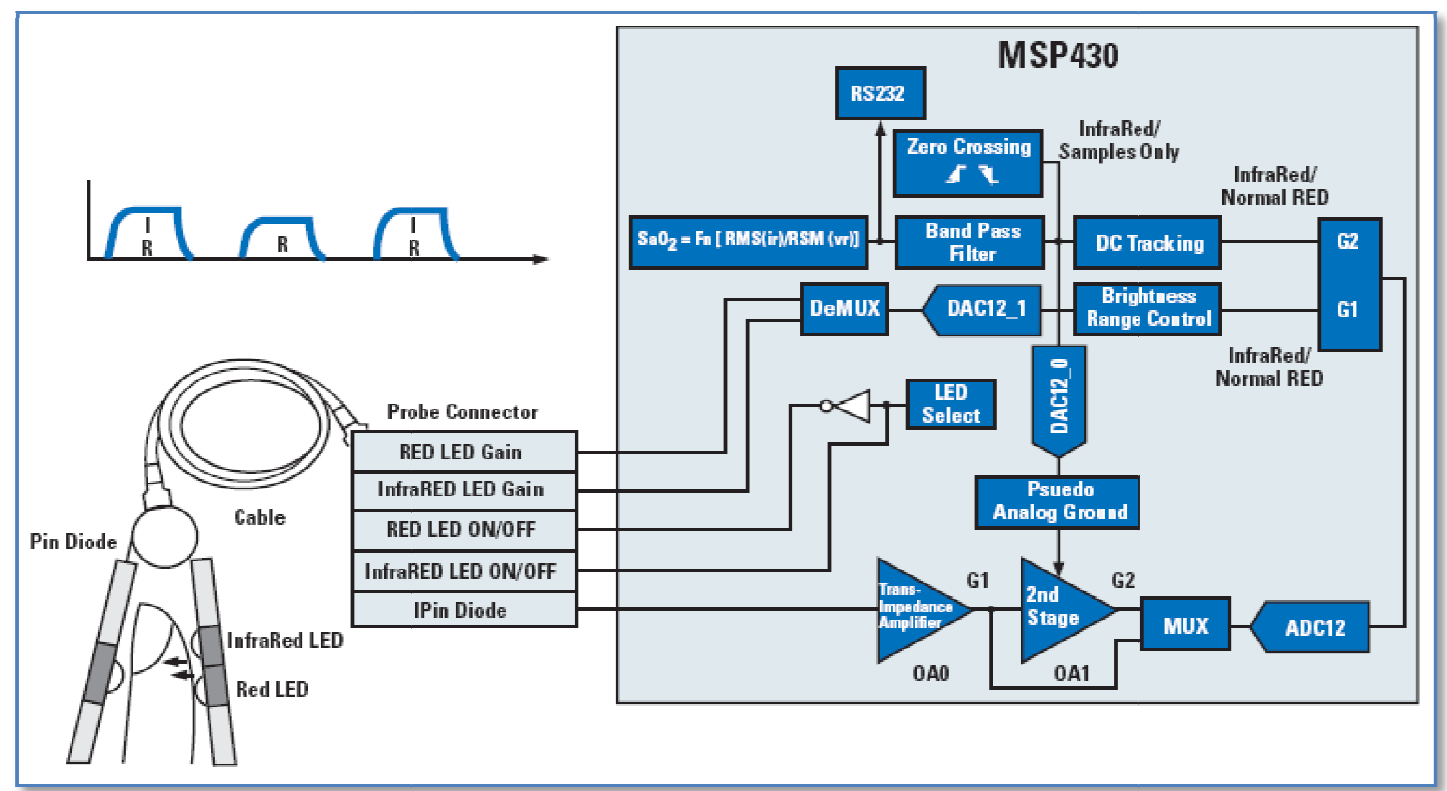

Figure 5-4 Block diagram of pulse oximeter system [44]

\section{Background and Implementation of Pulse Oximeter Measurement}

Oxygen saturation will be monitored using a non-invasive pulse oximeter on the index finger of the patient. The pulse oximeter probe uses two LEDs on one side of the finger at wavelengths of $660 \mathrm{~nm}$ (red spectrum) and 905nm (infrared spectrum) respectively, and a photodiode on the other side of the finger, as illustrated in Figure 5-5. From the ratio of absorption of the infrared light and red light detected by the photodiode, the oxyhemoglobin and deoxyhemoglobin ratio can be calculated. The pulse oximeter gives a percentage of arterial hemoglobin in the oxyhemoglobin configuration. A reading of oxygen saturation in the range of $90 \%$ to $100 \%$ will be acceptable for a healthy person. A reading of less than $90 \%$ will indicate that the patient's oxygen saturation levels are too low and an alarm will be activated.

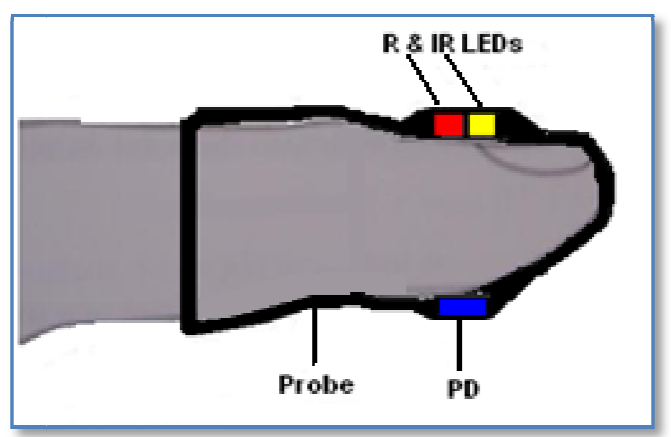

Figure 5-5 Pulse oximeter finger probe LEDs and PD placement 
Pulse oximetry relies on the recognition of a PPG signal as illustrated in the AC part of Figure 5-6. This signal is caused by changes in the arterial volume related with periodic contraction of the heart during systole. The magnitude of this signal depends on the quantity of blood ejected from the heart into the peripheral vascular bed with each cycle, the optical absorption of the blood, the composition and colour of the skin and underlying tissue, and the wavelengths used to illuminate the blood. $\mathrm{S}_{\mathrm{a}} \mathrm{O}_{2}$ is derived by analysing the magnitude of the red and infrared photoplethysmogram, measured by the photo detector. Inside the MCU the pulsatile (AC) and non-pulsatile (DC) components are calculated for the red and infrared photoplethysmogram as illustrated in Figure 5-6.

An algorithm contained by the pulse oximeter performs a mathematical naturalization by which the AC signal at each wavelength is divided by the related DC component that is mainly due to the light absorption by the bloodless tissue, residual arterial blood when the heart is in diastole, venous blood, and skin pigmentation. Since it is assumed that the AC portion in the photoplethysmogram results only from the pulsatile arterial blood component, this scaling method provides a normalized $\mathrm{red} /$ infrared ratio $\mathrm{R}$ which is highly dependent on the colour of the arterial blood and is therefore related to $\mathrm{S}_{\mathrm{a}} \mathrm{O}_{2}$ but is mainly independent of the volume of arterial blood entering the tissue during systole, skin pigmentation, and thickness. Therefore the instrument does not need to be recalibrated for measurements on different patients because of the above independent variables [44].

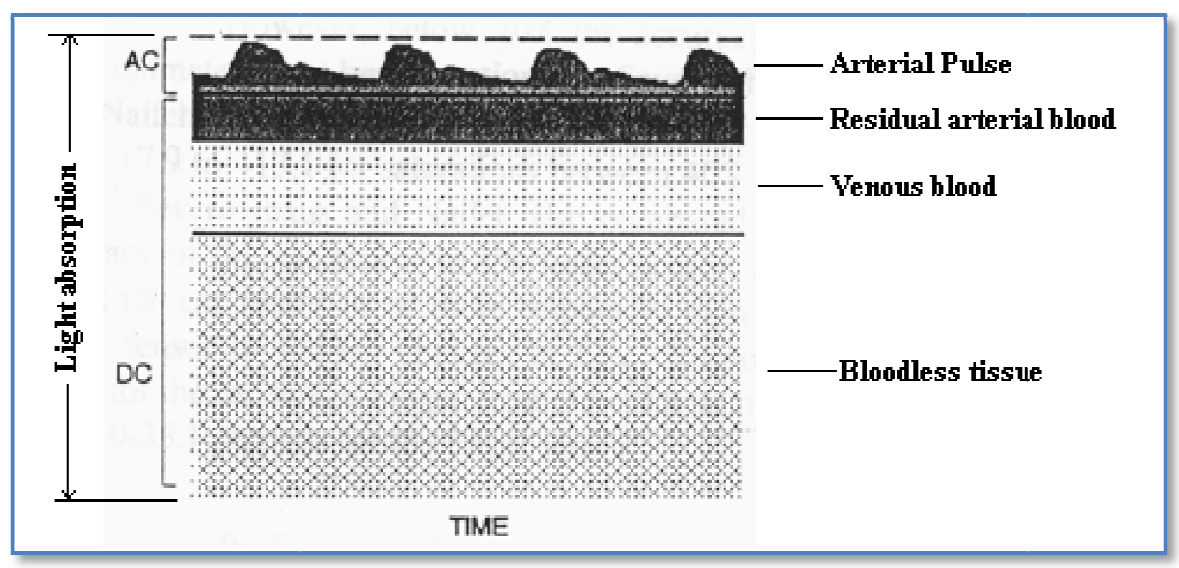

Figure 5-6 Time dependence of light absorption

Since both LEDs are pulsed, traditional analogue signal processing has to be abandoned in favour of digital signal processing. The signal samples are lowpass filtered in order to remove the $50 / 60 \mathrm{~Hz}$ noise. For each wavelength of light, the DC value is removed from the signal leaving the AC component of the signal, which reflects the arterial oxygenation level. The root mean square (RMS) value is 
calculated by averaging the square of the signal over a number of heartbeat cycles. The DC measurement is constantly calculated by averaging the signals over a number of heartbeat cycles. The driving strength of each LED is controlled, so that the DC level seen at the PIN diode meets a set mark level with a small tolerance. By doing this for each LED, the final result is that the DC levels of these two LED match each other to within a small tolerance. Once the DC levels match, R is calculated by dividing the logarithms of the RMS values, as shown in equation 5.1.

$$
R=\frac{\log \left(I_{a c}\right) \lambda_{1}}{\log \left(I_{a c}\right) \lambda_{2}}
$$

Where $\lambda_{1}$ and $\lambda_{2}$ represents the two different wavelengths of light used [44].

The most common problems with photoplethysmographic sensors are due to its use of optical sensors. These optical sensors are susceptible to motion artefacts. Psychotic patients will not always be still during measurements, and remaining still for an extended period is necessary for accurate measurements. Thus, it is reasonable to expect that motion artefacts will be introduced. The motion can be detected with an accelerometer and will assist in knowing when to take measurements. Measurements will only be taken when the arm is not moving above a predetermined range of accelerations.

\section{Calculation of the $\mathrm{S}_{\mathrm{a}} \mathrm{O}_{2}$ Calibration Curve}

In this application an oxygen saturation level of less than $90 \%$ will indicate that the patient requires medical attention and an alarm will be sounded when this threshold is reached. The M1190A oxygen saturation probe from Hewlett Packard (HP) is used on the index finger [45].

In practice, the relationship between $\mathrm{S}_{\mathrm{a}} \mathrm{O}_{2}$ and $\mathrm{R}$ is not linear due to scattering effects, complicated tissue structures and non-ideal light sources. For this reason the ratio value was calibrated using an empirical calibration curve.

A cohort study was done for HP by Severinghaus [46] to calculate the oxygen saturation probes calibration curves. The calibration curve calculated by HP for the HP M1190A (Figure 5-7) was implemented in this project [46]. 


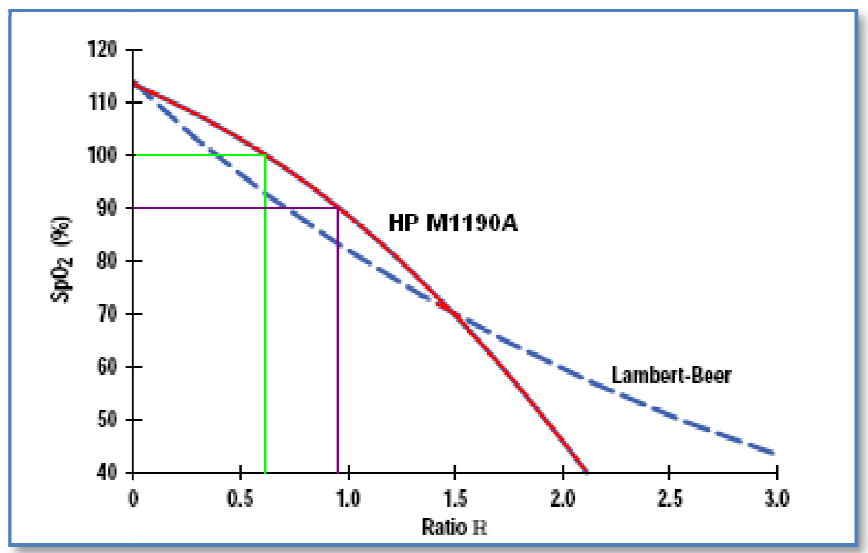

Figure 5-7 Empirical calibration curve used [45]

\section{Digital Switching of LEDs}

To use one photodiode to sample the red and infrared light, a sequential switching method should be used. The MCU is programmed to use Timer A to turn the two LEDs on and off in a sequential manner and to synchronously measure the photo detector output when the related LEDs are turned on. To implement this switching of signals, an H-bridge configuration was used. The $512 \mathrm{~Hz}$ switching frequency is calculated by using the digitally controlled oscillator (DCO). Figure 5-8 shows the circuit setup to implement the switching of the LEDs.

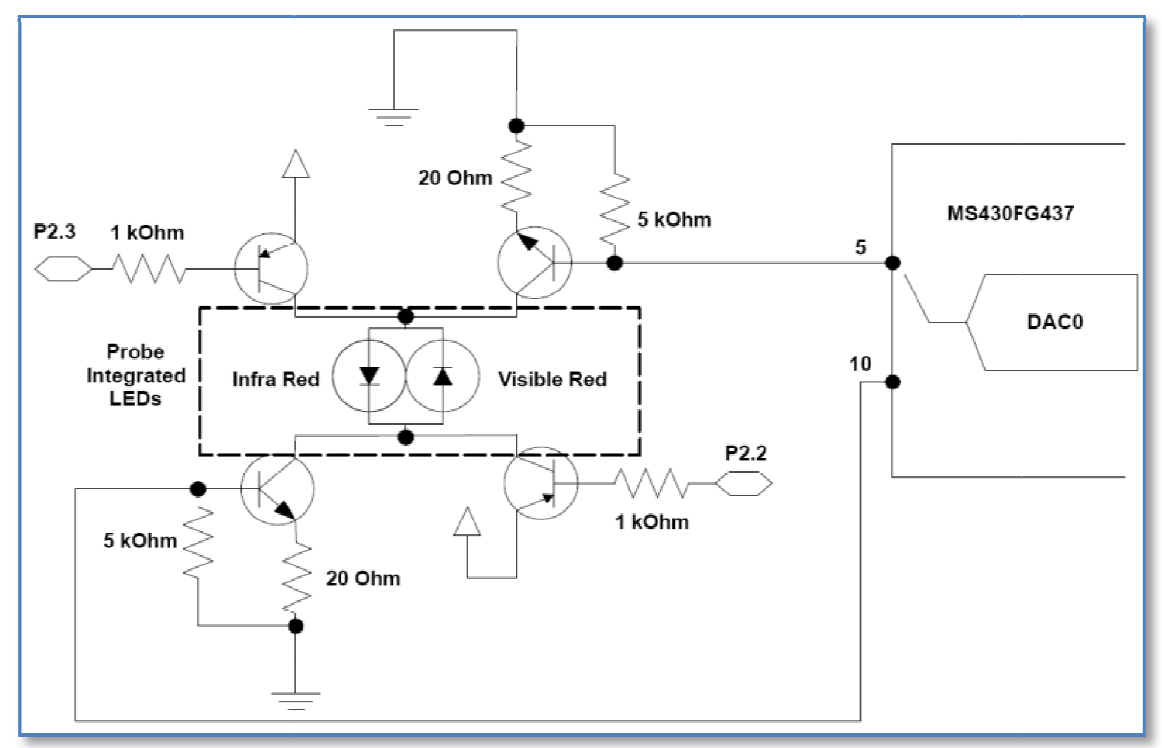

Figure 5-8 H-bridge circuit for digital switching of LEDs [44] 
DEVELOPMENT OF SAFETY GLOVE DEVICE

IV. Initial Prototype

The design was initially implemented with the development board. Figure 5-9 shows the developed initial prototype used to measure PPG graphs.

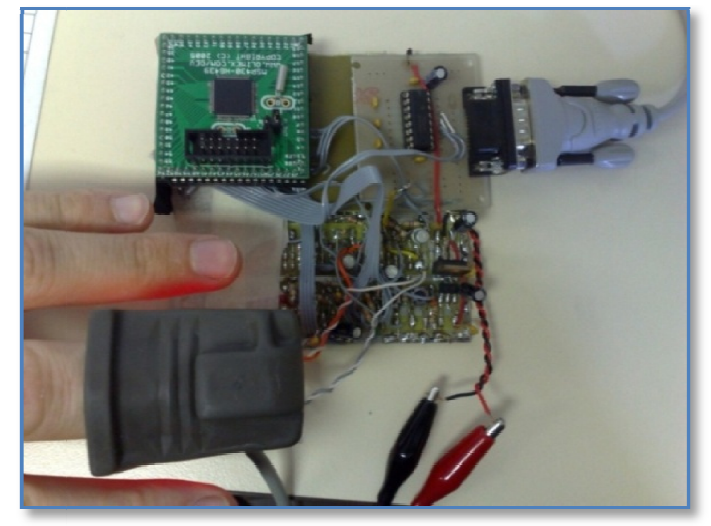

Figure 5-9 Initial prototype of pulse oximeter

\section{Plethysmograph Signals Measurement with Initial Prototype}

The initial prototype from Figure 5-9 was used to measure the two PPG signals. The two sampled signals were sent via serial cable to a PC and a software program supplied by Texas Instruments was used to display the sampled red (red) and infrared (blue) pulsatile signals. From these signals it is clear that the ratio can be calculated and that this prototype can measure the pulsatile signals. This initial prototype could then be developed further and implemented using a small PCB. The next section will explain the development of the PCB and will include an accelerometer to measure movement of the patient. Figure 5-10 shows the measured red and infrared signals.

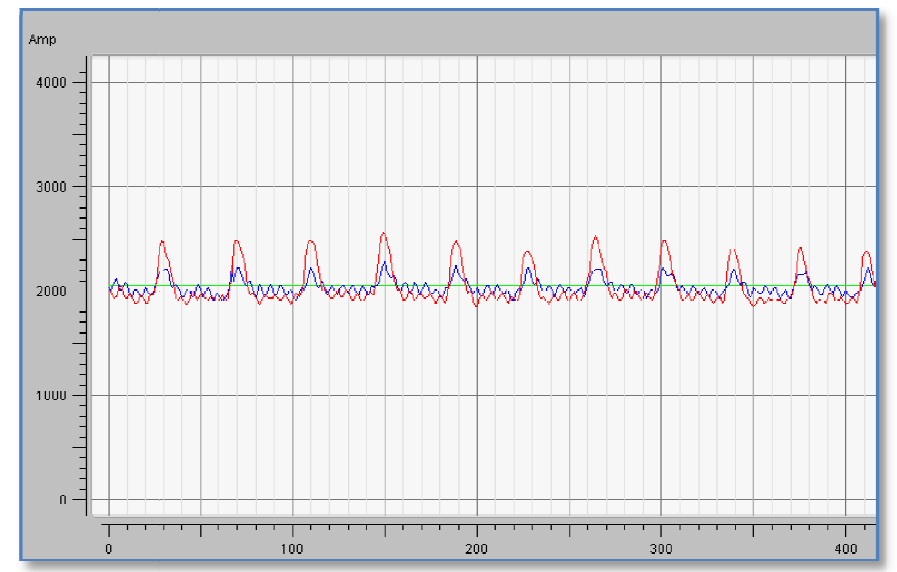

Figure 5-10 Display measured pulsatile red and infrared PPG signals 


\section{PCB Layout and Final PCB Prototype}

The size of the PCB should be a maximum of $60 \mathrm{~mm}$ by $40 \mathrm{~mm}$ to fit into the enclosure. The same 3.3V voltage regulator and Bluetooth module as in Section 4.4.2 were used. For PCB board layout for manufacturing of the PCB, refer to Appendix B2.

\section{Accelerometer}

The intention of an accelerometer in the wrist worn pulse oximeter is to monitor movement of the wrist. Any movement will influence the measurement of oxygen saturation, known as motion artefacts.

The ADXL311 accelerometer was chosen and placed on the PCB. The ADXL311 is a $+-2 \mathrm{~g}$ dual-axis accelerometer manufactured by Analog Devices [47]. According to the datasheet the ADXL311 should be wired as shown in Figure 5-11. Because of the analogue output it is easy to connect this module to the ADC channels of the MCU.

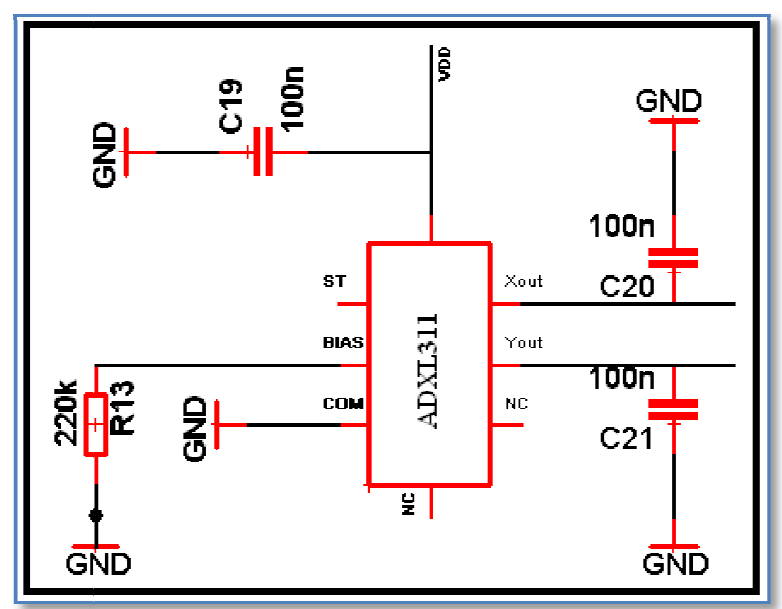

Figure 5-11 ADXL311 Accelerometer circuit diagram

\subsection{Microcontroller Software Design}

\subsubsection{Programming C-code}

The main code structure for the pulse oximeter measurement is obtained from Texas Instruments pulse oximeter design notes [43]. This code was used for the prototype. IAR embedded workbench version 3.42A from IAR systems was used to program 
and change the $\mathrm{C}$-code. Only the structuring and sending of data packages were changed in the original $\mathrm{C}$-code.

\subsubsection{Sending Serial Package Data}

For sending more than one channel of data over a serial connection, an identifier is needed to identify the beginning of a specific serial data package. The data package indicated in Figure 5-12 is a 1-byte red PPG data package. The identifier of this data package is a 1-byte $0 x F F$ hexadecimal value.

\begin{tabular}{|c|c|}
\hline 1 Byte & 1 Byte \\
\hline StartByte (0xFF) & PPG DATA \\
\hline
\end{tabular}

Figure 5-12 PPG data package

The saturation ratio value package is identified using a 2-byte identifier as shown in Figure 5-13.

\begin{tabular}{|c|c|c|}
\hline 1 Byte & 1 Byte & 1 Byte \\
\hline StartByte (0xFF) & StartByte (0xFF) & Ratio Value \\
\hline
\end{tabular}

Figure 5-13 Saturation ratio value package

The PPG data package is sent constantly while the saturation ratio value package is only sent at every fifth heartbeat. Figure 5-14 shows an example of possible serial data sending format having two PPG data packages, followed by a saturation ratio value package, followed by another PPG data package.

\begin{tabular}{|c|c|c|c|c|c|c|c|c|}
\hline 1 Byte & 1 Byte & 1 Byte & 1 Byte & 1 Byte & 1 Byte & 1 Byte & 1 Byte & 1 Byte \\
\hline SB & PPG & SB & PPG & SB & SB & Ratio & SB & PPG \\
\hline
\end{tabular}

Figure 5-14 Example of serial data format

\subsection{Mechanical Design}

\subsubsection{CAD Design and Selection of Electronic Protection Enclosure}

The PCB, two-cell battery pack, charging connectors and power switch should be fitted inside a protective enclosure. This enclosure was designed to be as small as possible and easy to manufacture. 


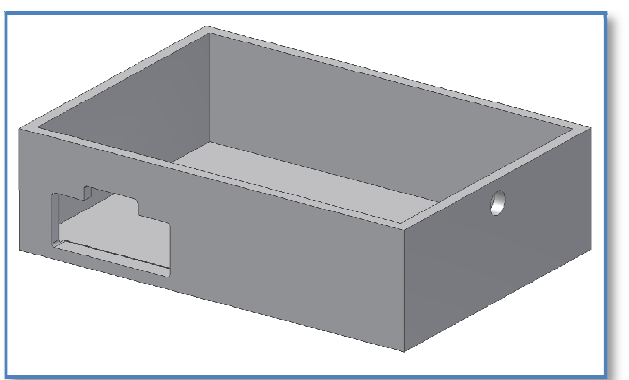

Figure 5-15 CAD design of electronic protection enclosure

\subsubsection{Battery Selection}

For this design, a generic form of the battery pack for a Nokia 8800 mobile phone was selected. The company that produces this generic battery only specifies the voltage and energy storage rating as $3.7 \mathrm{~V}$ and $650 \mathrm{mAh}$. Because of the low-dropout voltage of the $3.3 \mathrm{~V}$ voltage regulator, two of these Nokia 8800 batteries are used in series to produce an input voltage of $7.4 \mathrm{~V}$. The use of two of these batteries increases the storage size required inside the protective enclosure. Therefore the Nokia 8800 batteries were chosen, due to their small size. The drawback of this is the battery life. The batteries can only supply $650 \mathrm{mAh}$, which will be enough to power the safety glove device for at least 10 hours of continuous operation before a recharge is needed.

\subsubsection{Enclosure Selection}

The final prototype should have a protective enclosure manufactured out of a strong and lightweight metal to ensure the protection of the electronics. For the purpose of this thesis a commercial plastic enclosure was acquired that conformed to the requirements. Figure 5-16 shows the plastic enclosure with the two batteries, the charging connectors and power switch fitted inside.

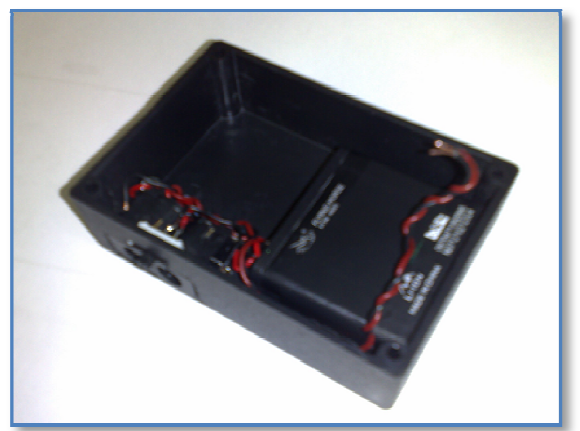

Figure 5-16 Plastic protective enclosure 


\subsection{Finalizing the Prototype}

\subsubsection{Combining Electronics and Mechanical Parts}

After the battery and the switches are implemented as illustrated in Figure 5-16, the PCB can be installed together with the onboard Bluetooth module. The wires of the oxygen saturation probe will also be attached through the hole to the PCB. The protective enclosure is closed and fastened with four screws, as illustrated in Figure 5-17.

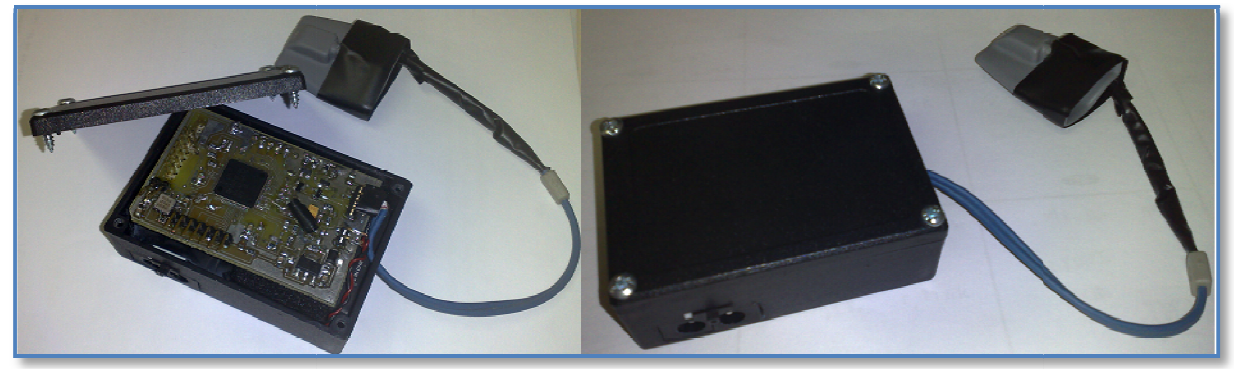

Figure 5-17 Protection enclosure with PCB installed and closed

\subsubsection{Proposed Positioning of the Device}

The prototype can be fitted inside a glove that will protect it from damage. However, for the purposes of this thesis the prototype enclosure is protected by a $3 \mathrm{~mm}$ rubber band. This rubber band is covered with blue nylon fabric velcro. A Velcro strap is used to attach the product around the wrist of the patient as show in Figure 5-18.

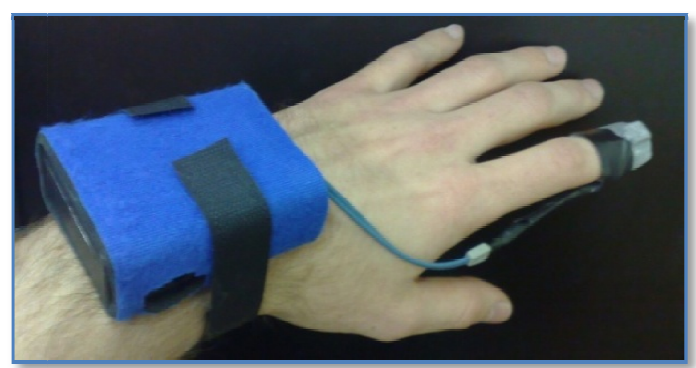

Figure 5-18 Velcro rubber protection and Velcro strap

\subsubsection{Final Prototype with the Glove}

The protective enclosure together with the wires and the finger probe can be covered with a glove to protect the device. In this prototype a latex glove was used to illustrate how the final product may work. The final product's glove should be designed with a rubber mould to ensure that the patient cannot destroy or damage the 
glove. The glove should also have a locking mechanism at the back to ensure that only the clinician can remove it. This locking mechanism was not designed but should be included in a commercial product. Figure 5-19 shows the latex glove, and this is also the final prototype for the safety glove. For measurement results using this device, refer to Chapter 7.

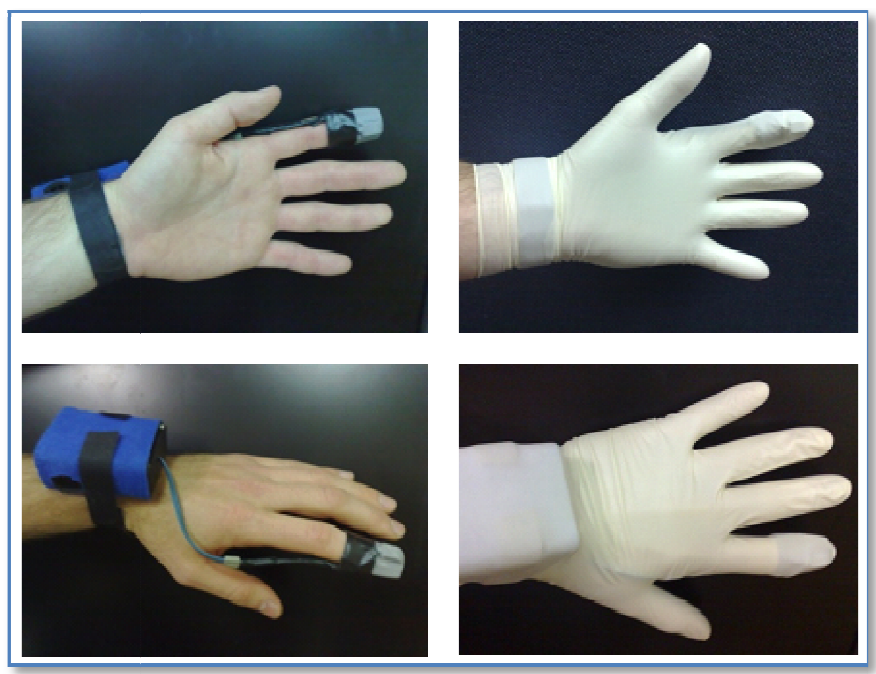

Figure 5-19 Final safety glove prototype

\subsection{Chapter Summary}

This chapter presented the development of the safety glove device. The use of a single chip pulse oximeter design was explained and the protective enclosure was designed. A final implementation of a prototype together with the placement of the device to the patient was shown. Section 5.5.3 shows the final prototype using a latex glove. The final prototype was used to sample the PPG signal in Figure 5-20.

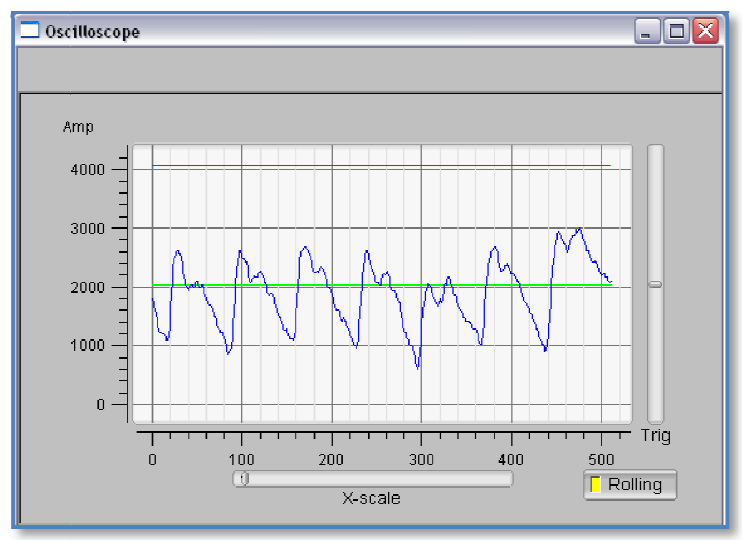

Figure 5-20 PPG signal from safety glove prototype 


\section{CHAPTER 6}

\section{GUI AND SIGNAL PROCESSING}

In this chapter the Matlab GUI will be explained in detail, while the methods used for heart rate detection, respiratory rate detection, warning signs, filters and algorithms are presented in the signal processing section.

\subsection{Matlab GUI}

Digital signal processing (DSP) can be computationally intensive. Matlab is optimised to perform these steps using predefined functions. There are some restrictions when using Matlab, for instance when sampling serial data and processing the data in real time. Matlab can only execute one instruction at a time, either storing serial data into an array or processing it. Due to this limitation it is not possible to monitor the patient continuously. The data is recorded for 20 seconds and then for a short interval of approximately two seconds the data is analysed for any warning signs. Then the relevant signals are displayed on the screen for the clinician to perform additional visual monitoring. The next 20 seconds are then recorded and the display is updated with the latest recorded data. Additional variables are used to add all the data together. When the user presses the "save data" button, he can select where to save these recorded variables on the hard drive for future reference. Figure 6-1 shows the developed GUI.

The first graph (1) displays the red light PPG signal obtained from the pulse oximeter on the index finger. The second graph (2) displays the ECG waveform for the first eight seconds. Graph 3 displays the HRV graph. HRV is the time between repeated heart beats. A constant graph with almost no variations will indicate that the heart rate is stable over time as shown in graph (3) in Figure 6-1. The HRV can help the clinician to monitor for sudden, uneven heart rates. The small bar graph (4) displays the level of movement. This level is calculated using the amount of g's that the accelerometer senses. This will give the clinician a good indication of whether the patient is resting or moving at uncontrollable rates. When the bar is full, the patient needs to be visited to ensure his/her safety. Graph 5 displays the filtered respiratory sound. Graph 6 will display the detected respiration of the patient for the last 20 seconds. 
At the top of the GUI the calculated heart rate, temperature, respiratory rate and $\mathrm{S}_{\mathrm{a}} \mathrm{O}_{2}$ values are displayed and updated every 20 seconds. All this information is displayed to the clinician on duty to ensure that he/she can investigate the warnings by visual inspection of the displayed graphs. The warning sign levels can be changed for each of the vital signs using the text box provided for each sensor. These values should be changed before the user presses the "start receiving data" button.

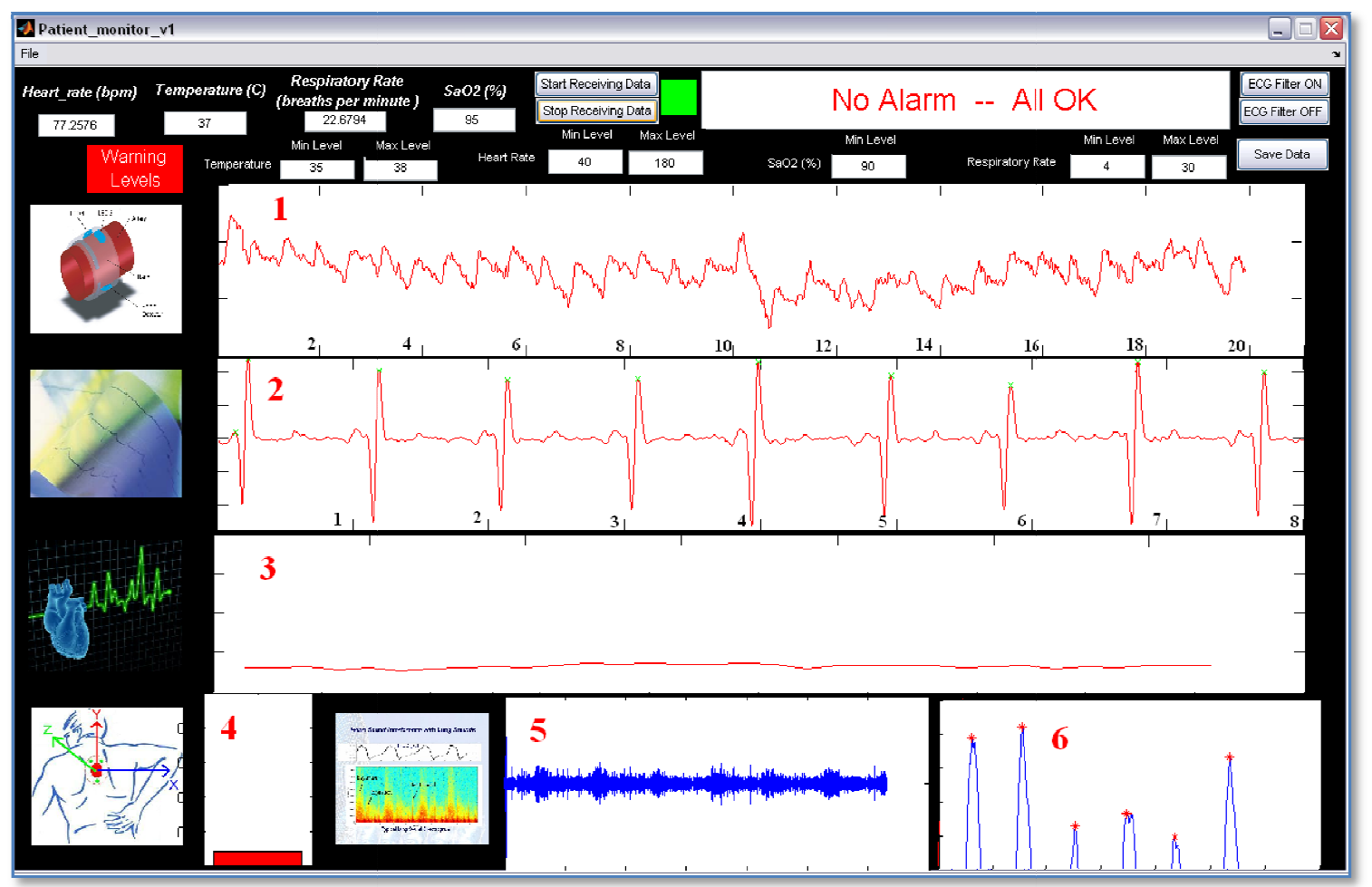

Figure 6-1 Matlab GUI of patient monitor system

\subsubsection{Program Flow}

The program flow structure for the sampling, plotting and monitoring of vital signs using a Matlab GUI is illustrated in the block diagram of Figure 6-2. 


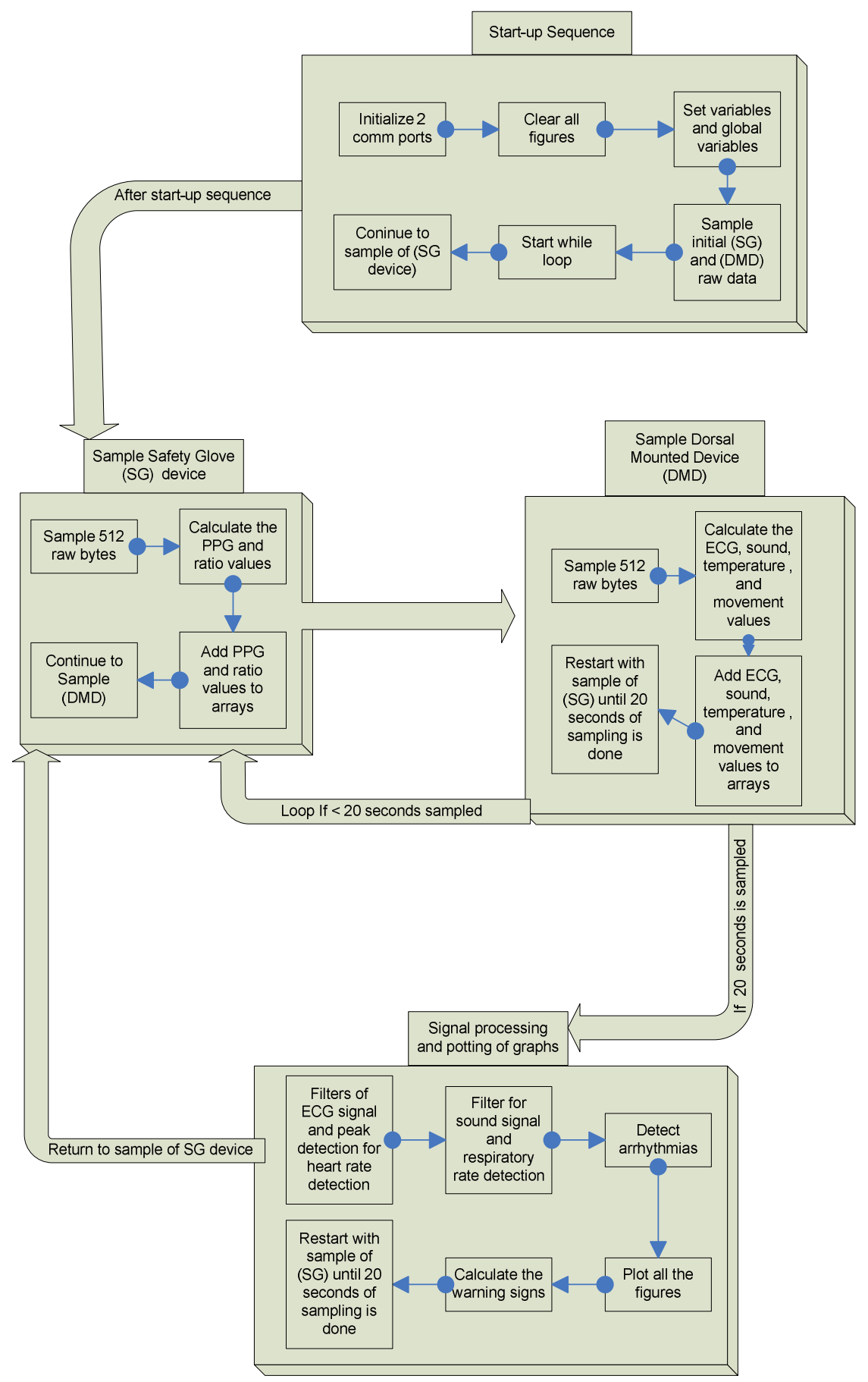

Figure 6-2 Matlab software program flow diagram 


\subsubsection{Communication Protocol}

\section{Dorsal Mounted Device Receiving Protocol}

The serial data sent by the MCU is received in Matlab using COM port 1. Matlab has a 512 bytes serial data buffer to store incoming serial data. If the data is not used before new data arrives at the serial port, data will be lost. The GUI will clean the buffer into an array at a faster rate than receiving data to avoid losing data bits. The received serial data is then processed by the GUI to retrieve all the relevant information. An identifier (SB) is sent at the onset of each new data package to be able to extract the four sensor values. Figure 6-3 shows the structure of the data package used for the dorsal mounted device. The GUI will locate the first identifier of the 512 bytes received, and will then sort the rest of the data into five new arrays for the ECG, sound_H, sound_L, temperature and movement. The sound_L and sound_H bytes are then converted to one 12-bit sound variable.

\begin{tabular}{|c|c|c|c|c|c|c|c|c|c|c|c|}
\hline 1 Byte & 1 Byte & 1 Byte & 1 Byte & 1 Byte & 1 Byte & 1 Byte & 1 Byte & 1 Byte & 1 Byte & 1 Byte & 1 Byte \\
\hline SB & Temp & ECG & S_H & S_L & Acc & SB & Temp & ECG & S_H & S_L & Acc \\
\hline
\end{tabular}

Figure 6-3 Dorsal mounted device data package received from MCU

\section{Safety Glove Device Receiving Protocol}

The serial data sent by the safety glove's MCU is received in Matlab using a virtual COM port. The GUI should be set up to sample from this virtual COM port. The GUI will clean the safety glove serial port buffer into an array at a faster rate than receiving data to avoid losing data bits. The serial data structure is almost the same as that of the dorsal mounted device. One identifier (SB) is used at the onset of each new PPG data package to be able to extract the PPG value. The oxygen saturation ratio value has two identifiers (SB) before the ratio value. Figure 6-4 shows the data structure of the data package used for the safety glove device. The GUI will locate the first identifier of the 512 bytes received and will then sort the rest of the data into two new arrays for the red PPG graph and the oxygen saturation ratio values. The PPG1 to PPGN values are stacked into an array to obtain the original PPG sampled graph.

\begin{tabular}{|c|c|c|c|c|c|c|c|c|}
\hline 1 Byte & 1 Byte & 1 Byte & 1 Byte & 1 Byte & 1 Byte & 1 Byte & 1 Byte & 1 Byte \\
\hline SB & PPG 1 & SB & PPG 2 & SB & SB & Ratio 1 & SB & PPG 3 \\
\hline
\end{tabular}

Figure 6-4 Safety glove device data package received from MCU 


\subsubsection{Warning Alarms}

A psychotic patient in seclusion is at risk of death or injury. Therefore the clinician on duty should be warned by the system if any of the monitored vital signs move away from the pre-established limits. The warning levels are user selectable, because different patients will have different vital sign levels. The levels may vary by age, gender and fitness levels. Table 6-1 shows the general default warning levels that will be used to sound an alarm if the clinician does not change the levels to suit the currently monitored patient.

Table 6-1 Default warning levels

\begin{tabular}{|l|c|c|}
\hline & Minimum & Maximum \\
\hline Temperature $\left({ }^{\circ} \mathrm{C}\right)$ & 35 & 39 \\
\hline Heart rate $(\mathrm{b} p m)$ & 40 & 160 \\
\hline Respiratory rate $(/$ minute) & 4 & 40 \\
\hline Oxygen saturation (\%) & 90 & --- \\
\hline
\end{tabular}

\subsection{Signal Processing}

In this section various signal processing techniques will be discussed, such as digital lowpass and bandpass filters, a peak detection algorithm for heart rate and respiratory rate detection and detection algorithms for detecting arrhythmias from the ECG signal.

\subsubsection{ECG Signal Analysis}

In order to detect the patient's heart rate (HR), HRV and possible arrhythmias, a clean ECG signal is needed, therefore lowpass filters are used to clean the raw ECG signal of unwanted noise. After the filtering of the ECG signal the HR, HRV and arrhythmias algorithms will be discussed.

\section{Lowpass Filter}

A raw sampled ECG signal sampled on the dorsal part of the body has a relatively large SNR due to the distance that the electrical signal has to travel through the body to arrive at the back of the patient. The SNR is calculated using the average power of the signal, divided by the average power of the noise, as shown in equation 6.1: 


$$
S N R=\frac{P_{\text {Signal }}}{P_{\text {Noise }}}=\left(\frac{A_{\text {Signal }}}{A_{\text {Noise }}}\right)^{2}
$$

Figure 6-5 shows the spectrogram of a "raw" ECG using a surface plot. The relevant ECG signal information is located below $100 \mathrm{~Hz}$. For standard heart rate monitoring, frequencies of between $0.05-30 \mathrm{~Hz}$ are needed, but when different PQRST detection algorithms are used for diagnostic reasons, frequencies between $0.05-1000 \mathrm{~Hz}$ are needed [48]. Most of the noise is located in the higher frequency band above $100 \mathrm{~Hz}$. It can be seen from Figure 6-5 that all the relevant information of the ECG signal to detect heart rate is in the frequency band of 0 to $10 \mathrm{~Hz}$ for this design. Therefore the 3 $\mathrm{dB}$ cut-off frequency is selected to be $10 \mathrm{~Hz}$ for a first order lowpass Butterworth filter.

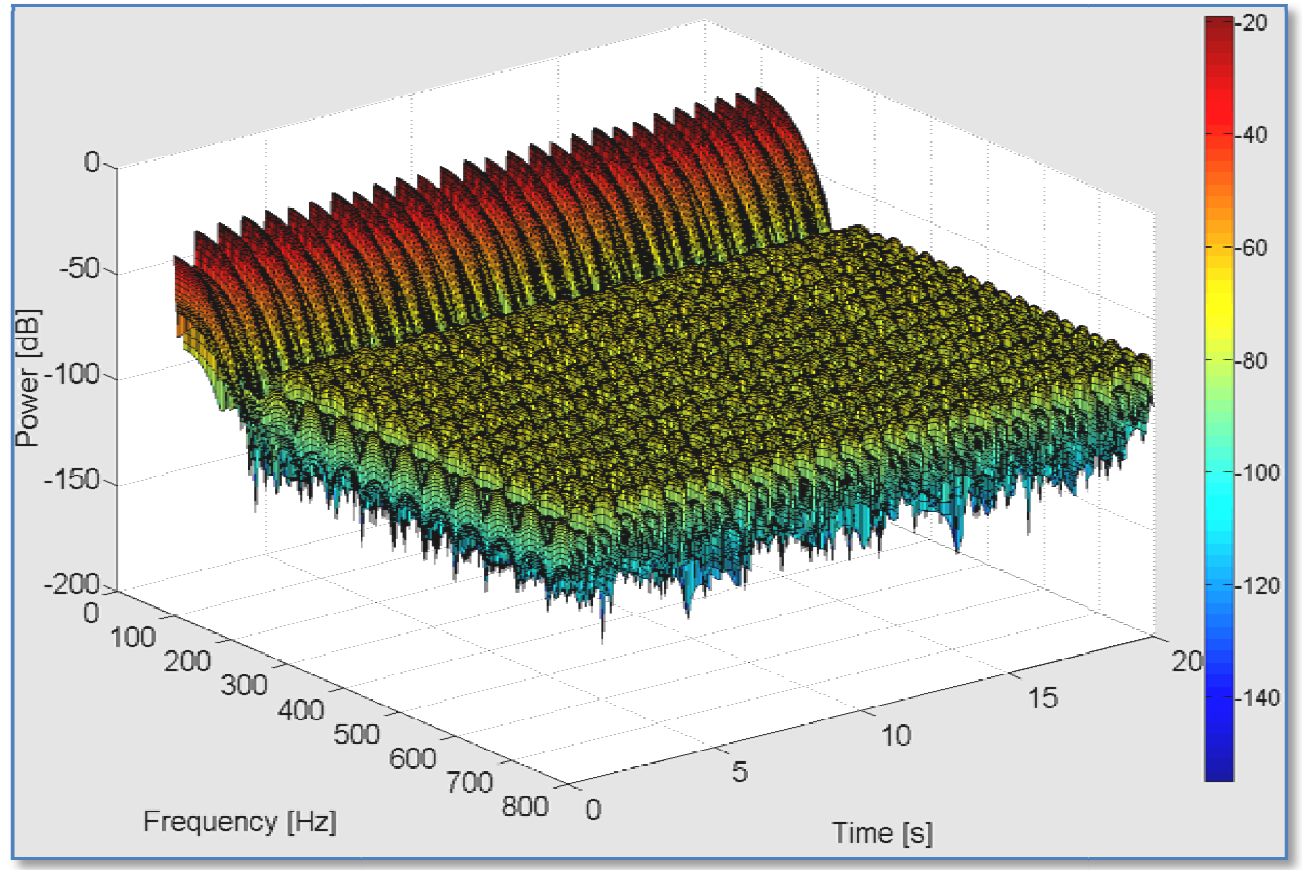

Figure 6-5 Spectrogram of raw ECG signal

A first order Butterworth lowpass filter is used to filter out all the high frequency noise. This enhances the SNR, as can be seen in Figure 6-6. The filter is used to clean the signal so that the PQRST characteristics can be identified from the signal, and peak detection can be implemented to obtain the R-R intervals. Figure 6-6 shows a sample of the raw ECG signal and the filtered ECG signal. 

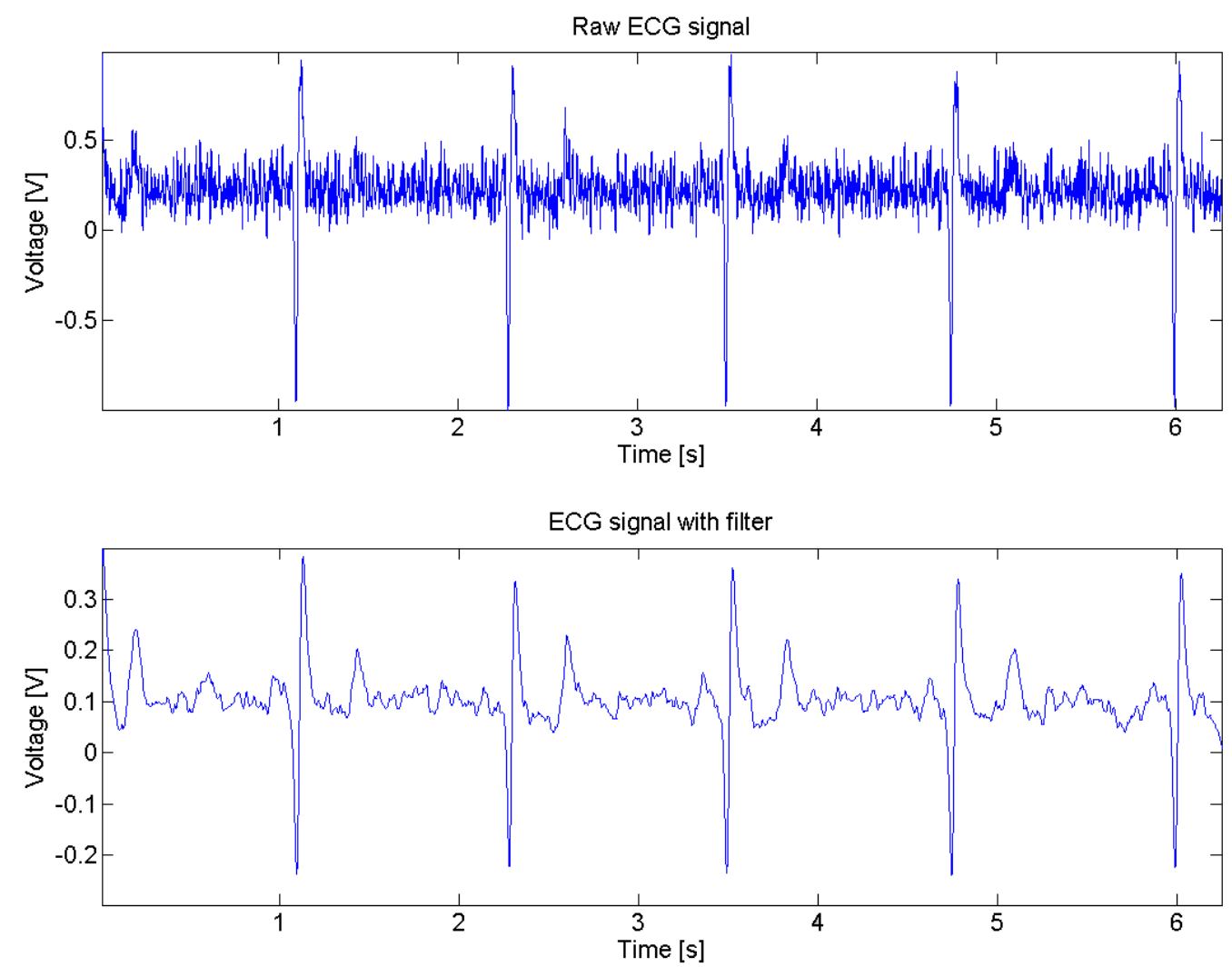

Figure 6-6 Raw and filtered ECG signal

\section{ECG Heart Rate Detection Algorithm}

Having a good ECG signal after filtering, the detection of the R peaks on the PQRST complex is done using a peak detection algorithm. In some cases the T component's amplitude can be large. This can lead to a false detection of the $\mathrm{T}$ component as an $\mathrm{R}$ component. This problem was overcome by developing a moving window peak detection algorithm. The peak detection algorithm detects a maximum point within the window and uses a threshold within that window. This ensures that there may not be more than one peak in a defined window at a time, erasing the $\mathrm{T}$ component from the detection. Figure 6-7 shows the time intervals between the $\mathrm{R}$ peaks. The average heart rate in beats per minute (BPM) can be calculated using equation 6.2:

$$
B P M=3 \times L
$$

with $\mathrm{L}$ the number of peaks detected in the 20 second sampling window. The heart rate will be calculated every 20 seconds. 

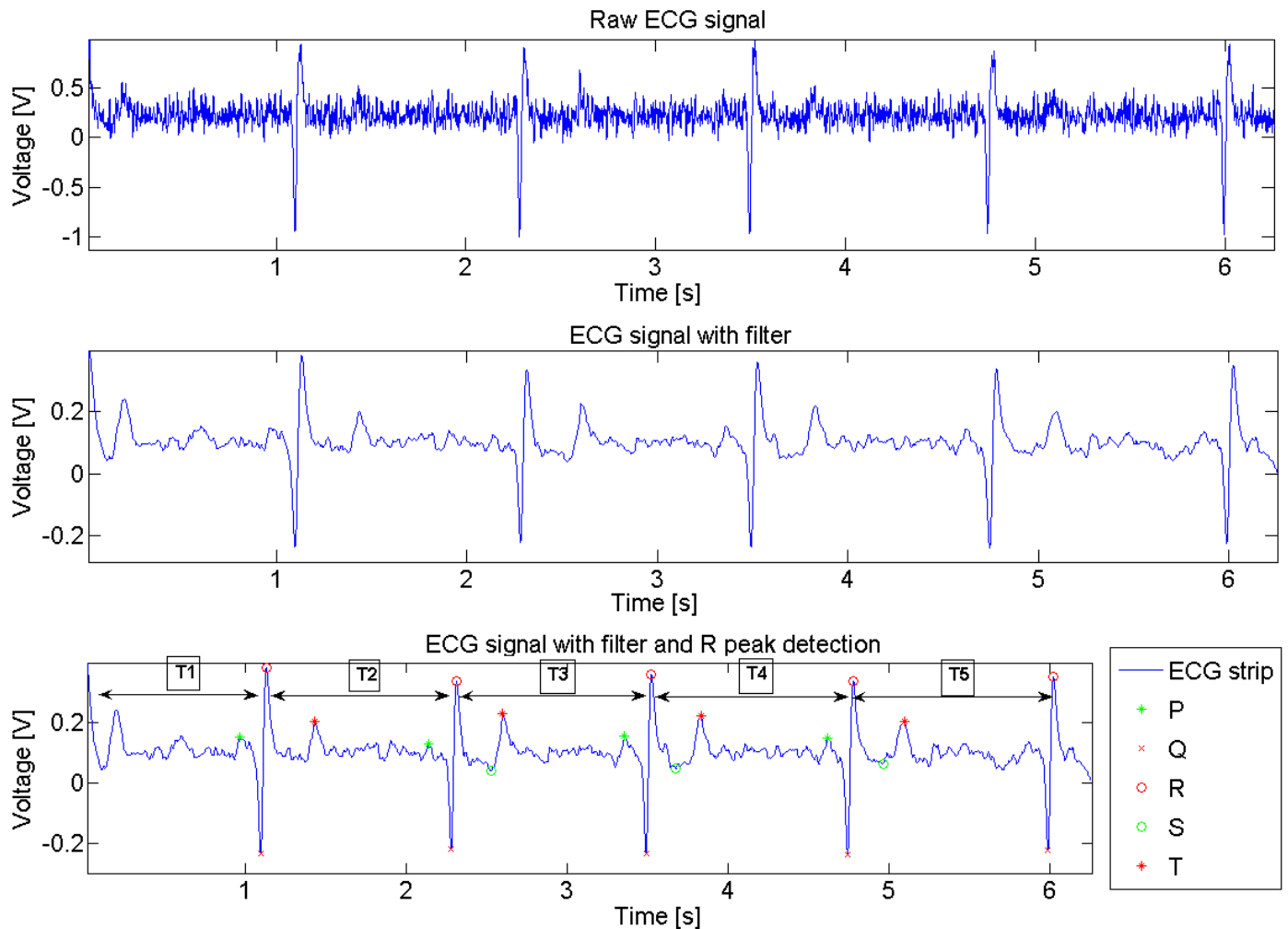

Figure 6-7 R-R interval and peak detection

III. ECG Heart Rate Variability Analysis and Arrhythmias Detection

The time between two R-R instants is defined as R-R intervals. Figure 6-8 shows the change in heart rate called the HRV for a 20 second sampling time. The interval number is the number of heart beats detected in the 20 second sampling time.

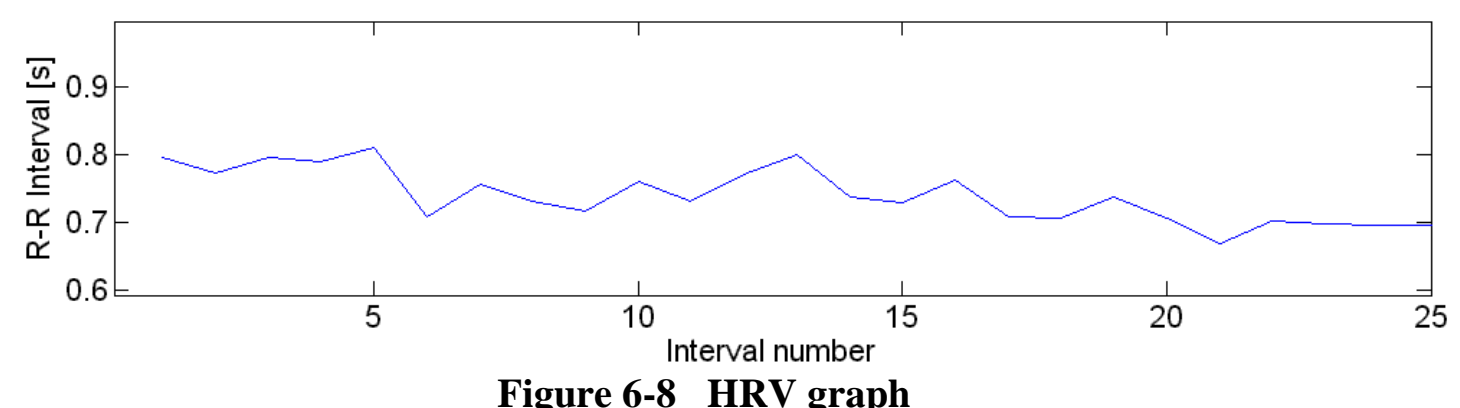

The detection of atrial fibrillation and premature ventricular contraction is made by means of large deviations of the HRV graph. 


\section{Atrial Fibrillation Detection Algorithm [49]}

There are two methods to detect atrial fibrillation. Firstly it can be detected by observing the P-waves in the ECG strip. When more than one P-wave occurs before the $\mathrm{R}$ peak, the patient has experienced an atrial fibrillation. The second characteristic of an atrial fibrillation is that the R-R intervals (HRV) will be inconsistent. It is observed as a "missing beat". The first characteristic is not possible to be calculated using the ECG waveform measured on the back of a patient, due to the unfavourable SNR. It was found that the SNR is not high enough to always obtain a useful PQRST waveform to detect more than one P-wave. The noise may influence the detection of the P-waves. Thus the second characteristic is used to calculate whether a possible atrial fibrillation has occurred. Figure 6-9 shows a simulated atrial fibrillation ECG strip.

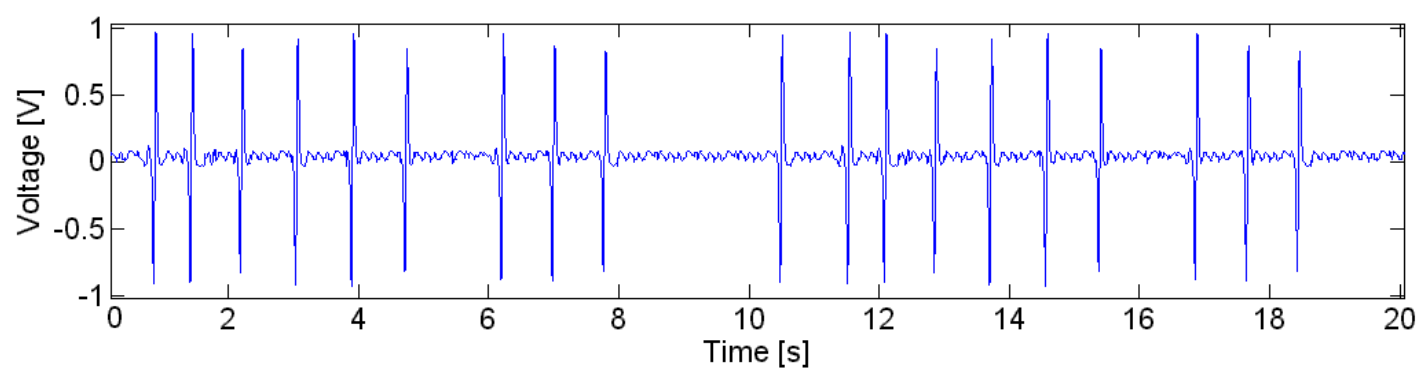

Figure 6-9 Simulated atrial fibrillation ECG strip

The HRV graph is used to detect a sudden change in heart rate. If the R-R interval (which is linked to HRV) changes suddenly with more than $50 \mathrm{~ms}$ above the average threshold it is classified as a "missing beat" and will then be recognized by the system as a possible atrial fibrillation (AF) [50]. For the sample HRV graph in Figure 6-10, three possible atrial fibrillation points are detected by the system.

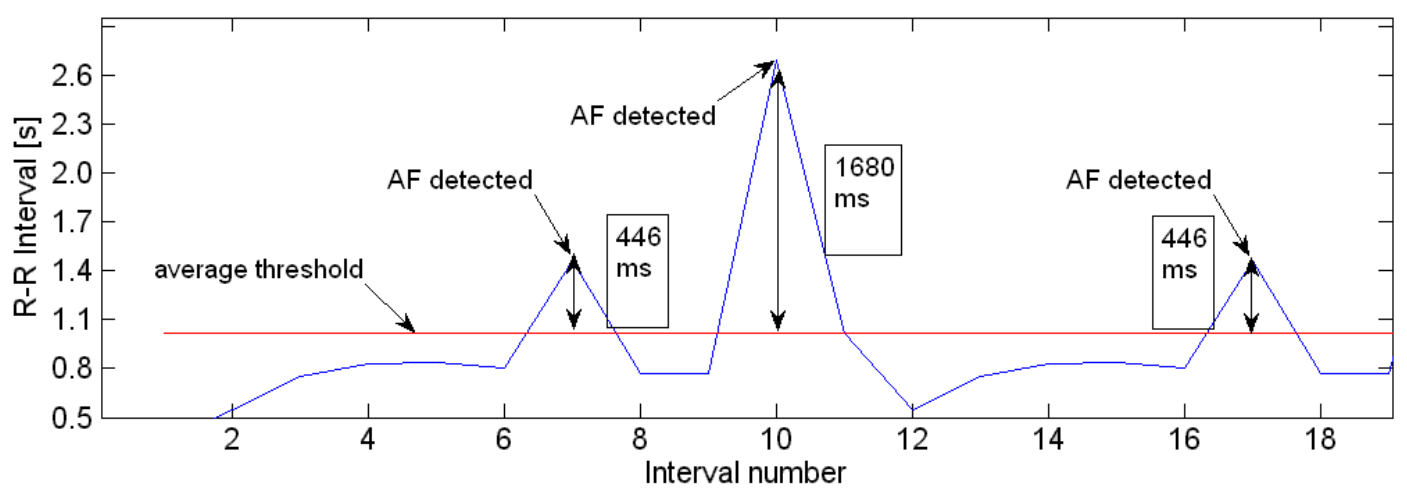

Figure 6-10 HRV graph with three atrial fibrillation points 


\section{Premature Ventricular Contraction (Extra systole) Detection Algorithm}

PVC occurs when an "extra" heartbeat is detected. The HRV is also used in this case for detection. When the HRV curve drops below the average threshold with more than $50 \mathrm{~ms}$, a possible PVC is detected [50]. Figure 6-11 shows an ECG simulation strip with PVC.

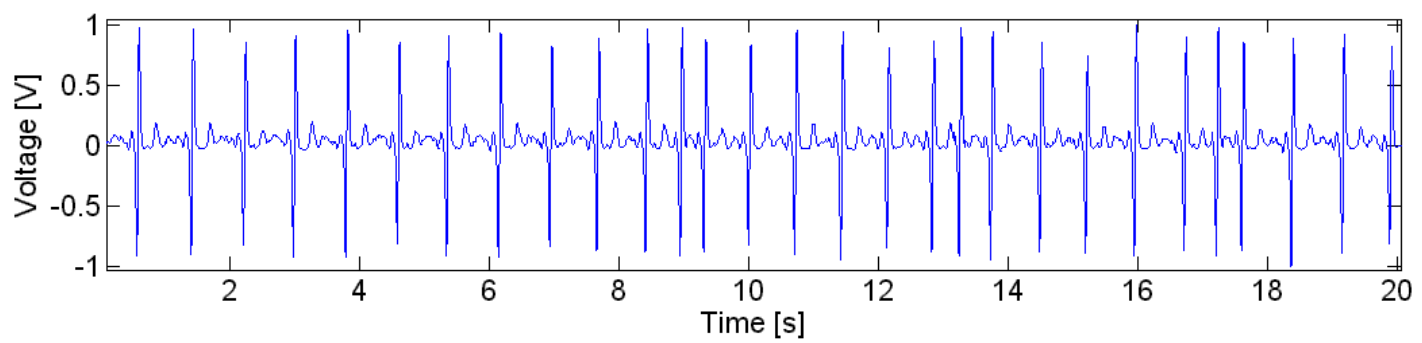

Figure 6-11 Simulated ventricular contraction ECG graph strip

Using Figure 6-11, the HRV graph is constructed and the detection of PVC is done using this HRV graph. Figure 6-12 shows the HRV graph with three extra systoles detected. The three extra systoles are $314 \mathrm{~ms}, 260 \mathrm{~ms}$ and $287 \mathrm{~ms}$ below the average threshold and are detected by the algorithm as PVC events.

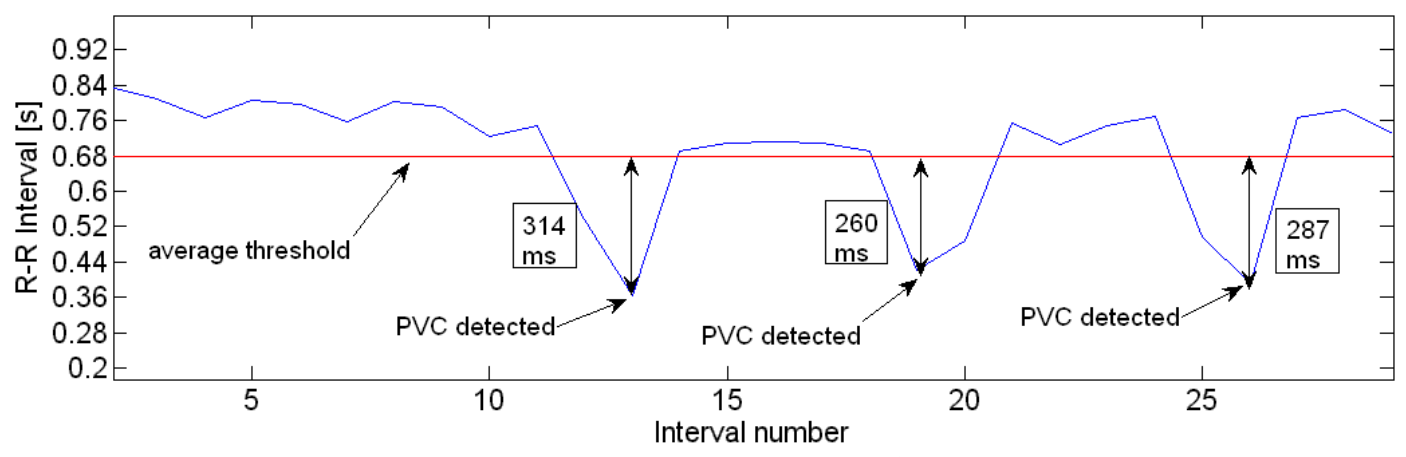

Figure 6-12 HRV graph with three PVC points detected

\subsubsection{Respiratory Rate Detection from Air-Coupled Microphone Signal}

The 12-bit $1600 \mathrm{~Hz}$ sampling frequency acoustic signal is used to calculate the respiratory rate of the patient. At the dorsal part of the body between the left and right scapula, the sound detection is very limited owing to the muscles between the microphone and the lungs. The recorded acoustic graphs display a lot of motion artefacts and noise. To ensure that a respiratory rate can be calculated, filters had to be developed to extract the respiratory sounds. Figure 6-13 shows a sample of the raw acoustic signal sampled for 20 seconds. 


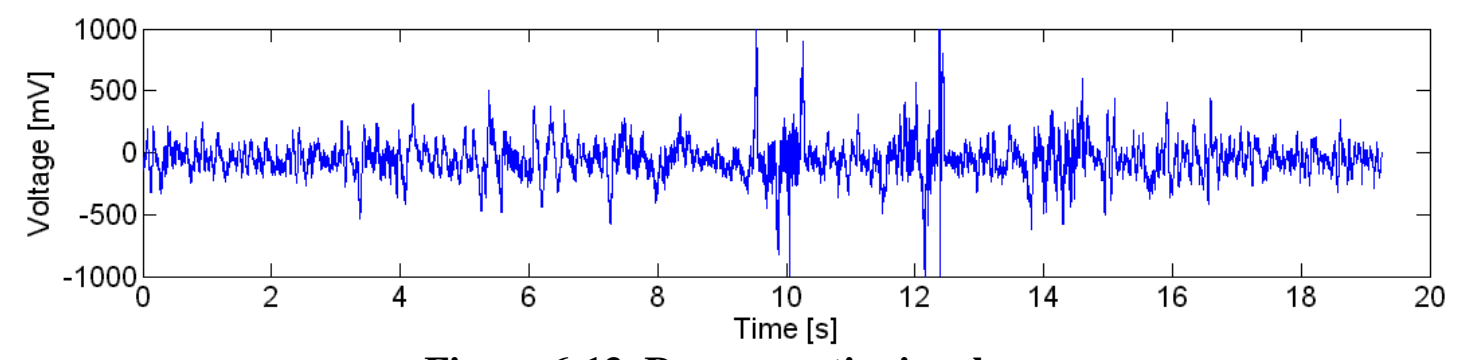

Figure 6-13 Raw acoustic signal

\section{Filters}

To extract the respiratory rate from the signal in Figure 6-13, the noise and motion artefacts must be removed. This is done using a bandpass filter. The frequency of the bandpass filter is calculated by investigating a spectrogram of the signal. After evaluating the spectrograms in Figure 6-14, it is clear the heart sounds are in the region of $0 \mathrm{~Hz}$ to $100 \mathrm{~Hz}$ and the main components of the lung sounds are in the frequency band of $187 \mathrm{~Hz}$ to $300 \mathrm{~Hz}$.

a)

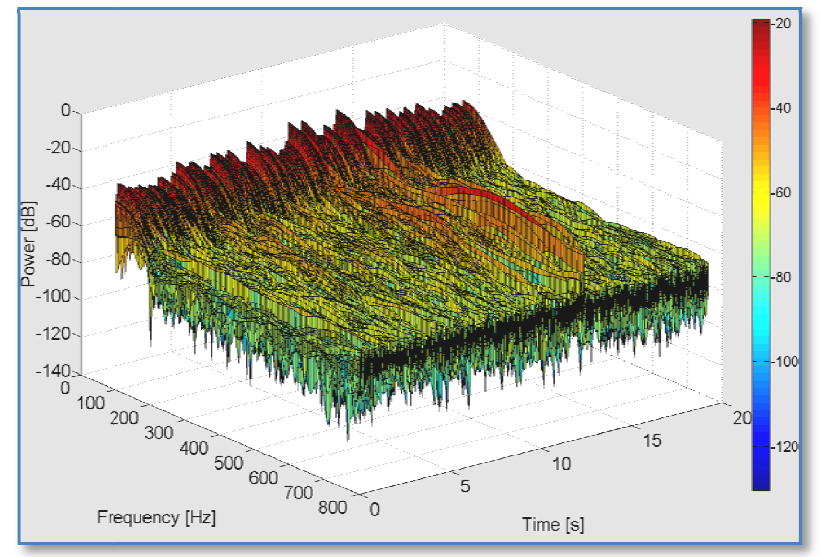

b)

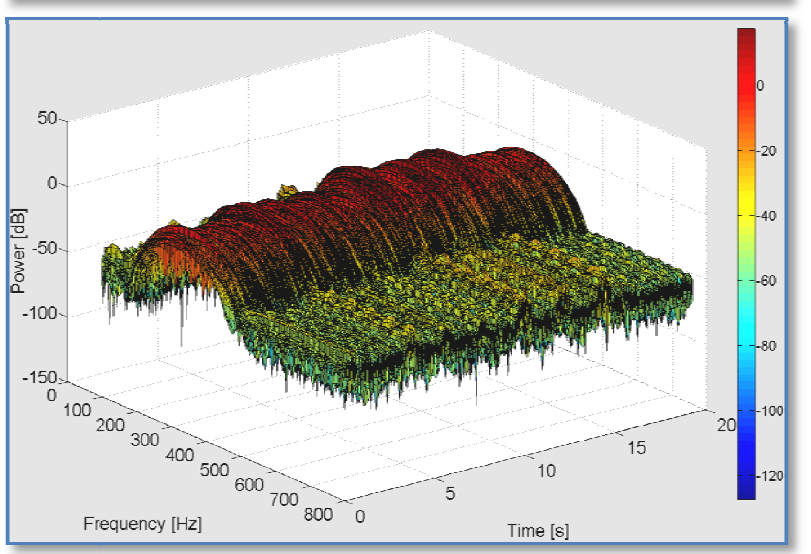

Figure 6-14 Spectrogram of acoustic signal 
The $10^{\text {th }}$ order Butterworth bandpass filter is developed to keep the frequency band of $187 \mathrm{~Hz}$ to $300 \mathrm{~Hz}$. Figure 6-15 shows the filtered acoustic signal revealing the respiratory sounds as desired.

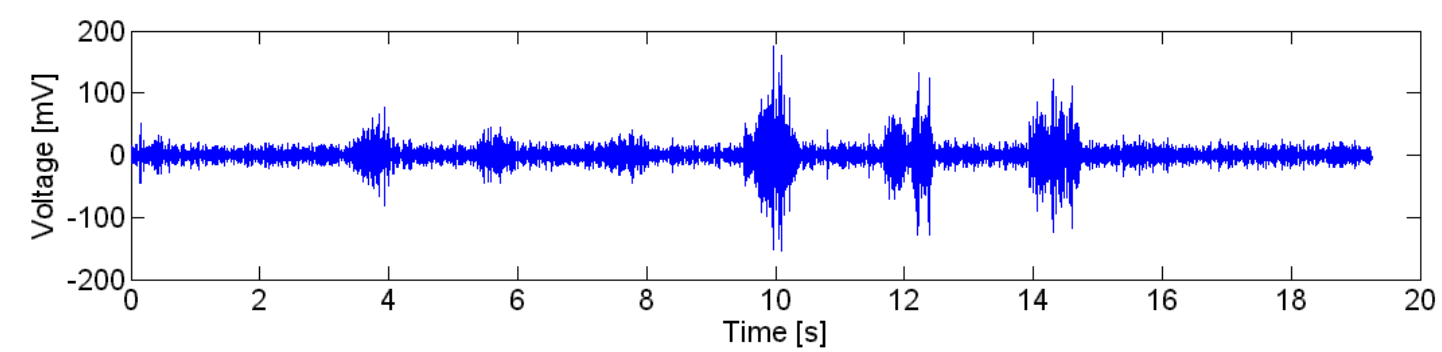

Figure 6-15 Bandpass filtered acoustic signal

Figure 6-15 shows three normal breaths and three deep breaths in a 20 second window. The inhalation is not visible due to the low SNR. Only the exhalation phase of one respiratory cycle can be seen, and this will be used to detect the respiratory rate of the patient.

\section{Respiratory Rate Detection Algorithm}

The filtered signal of Figure 6-15 is used to calculate the respiratory rate. A first order lowpass Butterworth filter with a $3 \mathrm{~dB}$ cut-off frequency of $1 \mathrm{~Hz}$ is used to detect the envelope of the signal. Figure 6-16 shows the results from the envelope filter used. The average of the envelope is used as a threshold for the peak detection. The last graph in Figure 6-16 shows the envelope above the calculated average threshold and with the peak detection algorithm implemented.

The peaks are then used to calculate the average respiratory rate (ARR) per minute using equation 6.3:

$$
A R R=3 \times L
$$

with $\mathrm{L}$ the number of peaks detected in the 20 second sampling window. The average respiratory rate will be calculated every 20 seconds using equation 6.3 . 

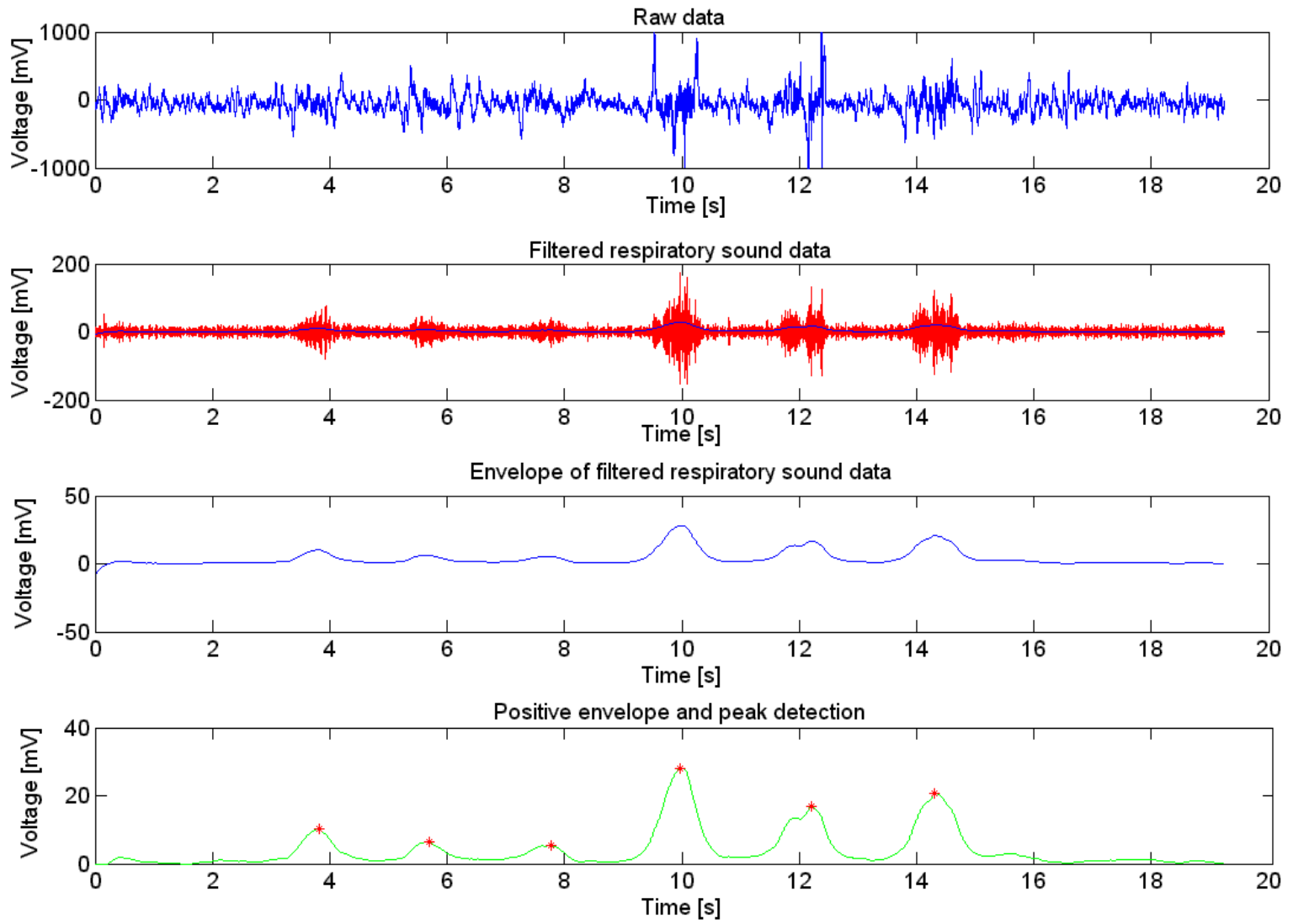

Figure 6-16 Raw, bandpass, envelope filtered and peak detection sound data

III. Respiratory Sinus Arrhythmia (validation of sensors correlation)

It is commonly known that the HRV changes during a breathing cycle. This change due to breathing is small compared to that of an extra systole or atrial fibrillation. Using the ECG signal for HRV detection and the filtered respiration signal envelope (Figure 6-17 and Figure 6-18), it can be seen that the heart rate variability changes relative to the breathing cycles. Thus the ECG and respiratory sensor correlates as expected.

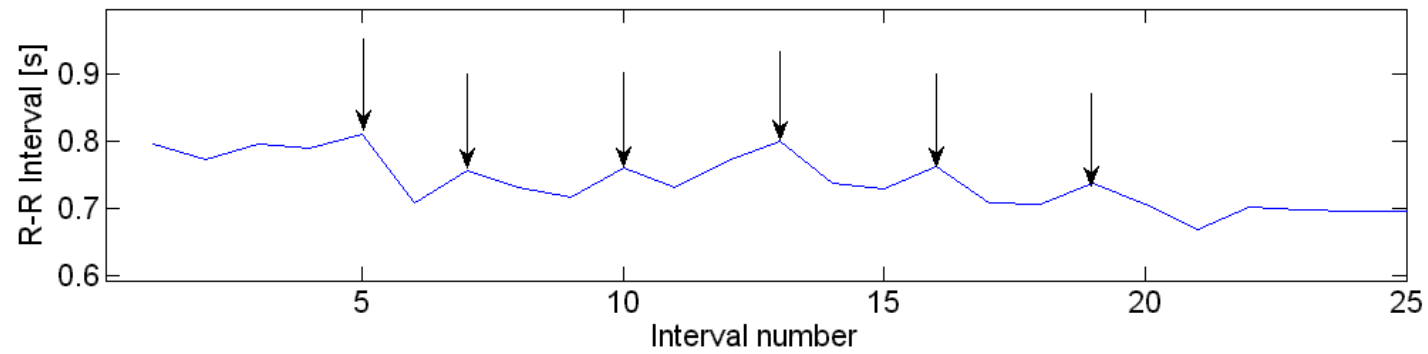

Figure 6-17 HRV graph 


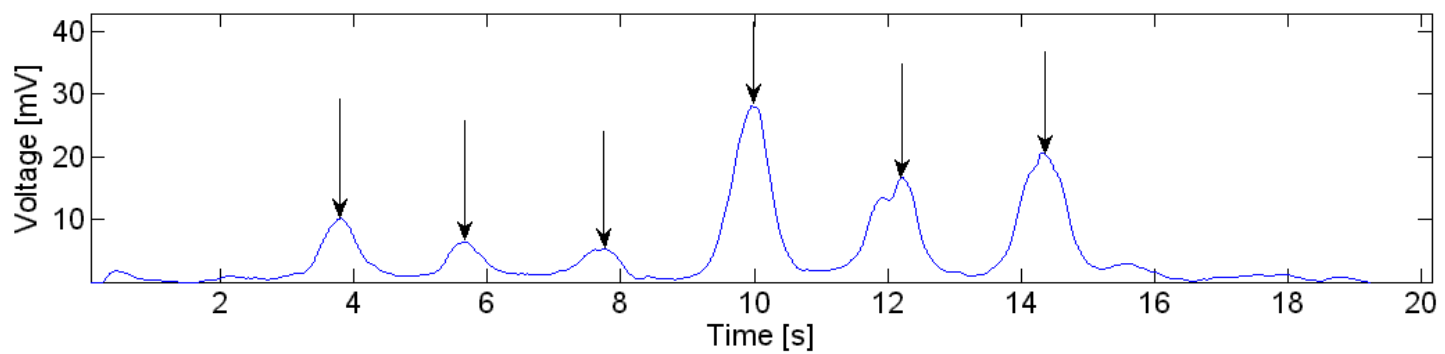

Figure 6-18 Respiration envelope

The heart rate is controlled by the cardiovascular centre within the medulla oblongata. Respiratory sinus arrhythmia (RSA) is a natural cycle of arrhythmia that occurs during the influence of breathing on the flow of sympathetic and vagus impulses to the sinoatrial node. The heart rhythm is primarily under the control of the vagus nerve, which inhibits heart rate and the force of the contraction. When a patient inhales, the vagus nerve activity is impeded and heart rate begins to increase. This pattern is reversed when the patient exhales. The heart rate's degree of fluctuation is also controlled significantly by regular impulses from the baroreceptors (pressure sensors) in the aorta and carotid arteries [51].

\subsection{Chapter Summary}

This chapter presented the central monitoring station which utilised a Matlab GUI. This GUI and each of the calculations and signal processing techniques used to detect vital signs were discussed. The physiological warning levels that are used to detect a medical emergency were explained and a block diagram of the program structure of the GUI was shown in Figure 6-2. Two arrhythmias detection algorithms were presented and simulated ECG strips were used to show the correct functioning of these detection algorithms. The use of a microphone to detect the respiratory rate was discussed with examples of measured data. 


\section{CHAPTER 7}

\section{TESTING AND RESULTS}

In this chapter, the laboratory testing setup is presented with the aid of pictures of the testing procedure. The results obtained from these tests, done on different volunteers, will be analysed and discussed.

\subsection{Laboratory testing}

Verification of all the sensors is required to ensure that the device is functioning as proposed. As it is difficult to measure changes in vital signs on psychotic patients in a psychiatric hospital, volunteers were asked to perform different activities to measure the changes in these variables. As discussed earlier, psychotic patients and healthy volunteers have the same types of vital signs. Due to this, healthy volunteers can be used to verify the measurements.

\subsubsection{Setup}

\section{Dorsal Mounted Device Setup for Testing Procedure}

The device was attached to a rubber band that was revolved around the volunteer's chest. This method is used to easily and quickly attach the device to the different volunteers. To validate the heart rate detection of the dorsal mounted device, the heart rate value will be compared with a commercial Polar T61 - coded heart rate monitor [52]. Figure 7-1 shows the test setup used and the Polar heart rate monitor attached to a volunteer.

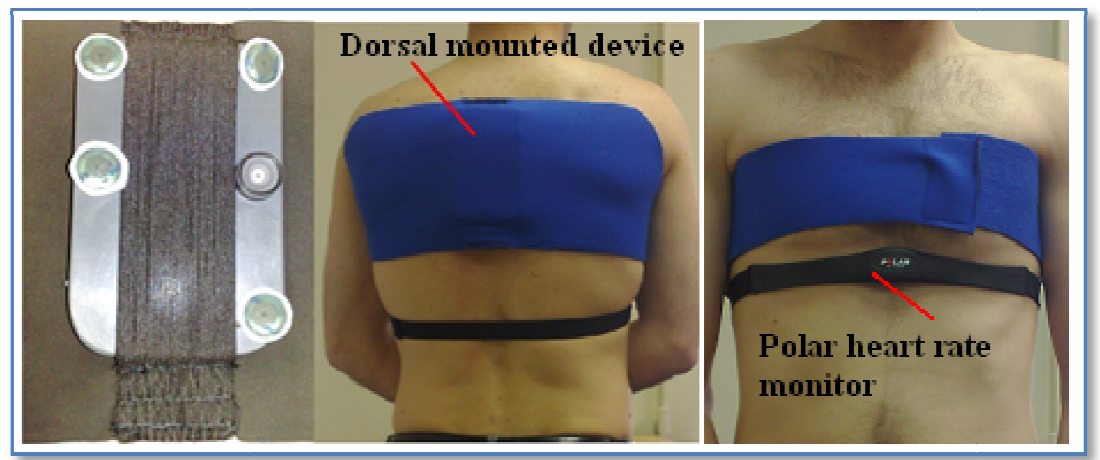

Figure 7-1 Prototype attached to a volunteer using a rubber band 


\section{Safety Glove Device Setup for Testing Procedure}

The safety glove was attached to the volunteer's wrist without the protective glove as indicated in Chapter 5. A Velcro strap was used to attach and remove the device around the wrist of the volunteer. This Velcro strap attaches the device firmly, but it will not impede blood flow during the testing procedure.

\subsubsection{Procedures for Validating Different Vital Signs}

Two tests are done on each volunteer to obtain changes in variables such as respiration detection, apnea, resting heart rate and excessive heart rate. The two tests are discussed in more detail below. In the first test the volunteer was asked to sit in a chair for two minutes and breathe normally in the beginning of the tests and three deep breaths in the middle of the test with a suspension of breathing at the end of the test. With Test 1 , the resting heart rate, normal breathing rate and suspension of breathing were measured. The average temperature and average oxygen saturation were also measured during Test 1 . In the second test the volunteer was asked to run up and down eight stairs until the measured heart rate was more than the predefined maximum heart rate. After this the patient was asked to sit in the chair again and the heart rate was monitored for another two minutes. With Test 2 the maximum heart rate was measured and the predefined alarm value for the excessive heart rate was tested.

I. Heart Rate Validation

- Test 1 - Sitting in a normal upright position to detect normal heart rate and breathing and $\mathrm{S}_{\mathrm{a}} \mathrm{O}_{2}$ :

A resting heart rate will be calculated using the ECG measurement.

- Test 2 - Do exercise to increase heart rate:

The volunteer should perform an exercise to increase the heart rate to a maximum for his age and weight. This test will indicate whether the GUI will detect if the volunteer's heart rate went above a predefined value.

II. Respiratory Rate Validation

- Test 1 - Sitting in a normal upright position to detect normal heart rate and breathing and $\mathrm{S}_{2} \mathrm{O}_{2}$ :

The volunteer should be breathing normally with three deep breaths in the testing period, followed by a suspension of breathing at the end of the testing period. 
- Test 2 - Do exercise to increase heart rate:

The volunteer may breathe normally during this exercise.

III. Temperature Validation

- Test 1 and 2 :

The temperature will be measured on the skin of the volunteer.

IV. Oxygen Saturation Validation

- Test 1 - Sitting in a normal upright position to detect normal heart rate and breathing and $\mathrm{S}_{\mathrm{a}} \mathrm{O}_{2}$ :

For the first test the volunteer should rest his hand on a table to ensure that the movement of the wrist is kept to a minimum. This will give the best readings for $\mathrm{S}_{\mathrm{a}} \mathrm{O}_{2}$ calculated from the PPG graphs.

- Test 2 - Do exercise to increase heart rate:

During the exercise test the volunteer can move his hand normally as if the device is not attached to his wrist. The accelerometer measurement will be tested and therefore no measurement of oxygen saturation will be taken due to the excessive movement of the wrist.

\subsection{Results of the Laboratory Testing}

The results obtained during the tests on the volunteers are summarised below. Table 7-1 summarises the physiological information of the volunteers.

Table 7-1 Volunteer physiological details

\begin{tabular}{|c|c|c|c|c|}
\hline Volunteer & Age & $\begin{array}{l}\text { Gender } \\
(\mathbf{M} / \mathbf{F})\end{array}$ & $\begin{array}{l}\text { Length } \\
(\mathbf{c m})\end{array}$ & $\begin{array}{l}\text { Mass } \\
(\mathbf{k g})\end{array}$ \\
\hline $\mathbf{1}$ & 24 & $\mathrm{M}$ & 180 & 83 \\
\hline 2 & 24 & $\mathrm{M}$ & 189 & 86 \\
\hline 3 & 24 & $\mathrm{M}$ & 183 & 86 \\
\hline 4 & 25 & $\mathrm{M}$ & 180 & 79 \\
\hline 5 & 26 & $\mathrm{M}$ & 174 & 79 \\
\hline
\end{tabular}

\subsubsection{ECG Measurement with Heart Rate Results}

An average heart rate was calculated over the two minutes of testing. These results are compared with those of the Polar - T61 heart rate monitor [52], as shown in Table 7-2. 
TESTING AND RESULTS

Table 7-2 Heart rate values from test data

\begin{tabular}{|c|c|c|c|c|c|c|}
\hline Volunteer & $\begin{array}{l}\text { HR } \\
\text { Test1 }\end{array}$ & $\begin{array}{l}\text { HR(Polar) } \\
\text { Test1 }\end{array}$ & $\begin{array}{l}\text { HIR } \\
\text { Test2 }\end{array}$ & $\begin{array}{l}\text { HR(Polar) } \\
\text { Test2 }\end{array}$ & $\begin{array}{l}\text { Difference } \\
\text { HR \% Test1 }\end{array}$ & $\begin{array}{l}\text { Difference } \\
\text { HR \% Test2 }\end{array}$ \\
\hline $\mathbf{1}$ & 69 & 70 & 140 & 140 & 1.5 & 0.0 \\
\hline $\mathbf{2}$ & 52 & 52 & 108 & 107 & 0.0 & 0.9 \\
\hline $\mathbf{3}$ & 65 & 67 & 163 & 165 & 3.1 & 1.2 \\
\hline $\mathbf{5}$ & 65 & 67 & 134 & 135 & 3.1 & 0.7 \\
\hline
\end{tabular}

From these results it is clear that the system can detect and calculate the heart rate accurately.

\subsubsection{Respiratory Rate Results}

The respiratory rate is calculated by counting the number of respirations in the first minute for Test 1 and Test 2 . These counted results are compared to those calculated by the system. These results are compared in Table 7-3.

Table 7-3 Counted vs. calculated respiratory rate

\begin{tabular}{|c|c|c|c|c|c|c|}
\hline Volunteer & $\begin{array}{l}\text { ARR } \\
\text { Test1 }\end{array}$ & $\begin{array}{l}\text { ARR(Counted) } \\
\text { Test1 }\end{array}$ & $\begin{array}{l}\text { ARR } \\
\text { Test2 }\end{array}$ & $\begin{array}{l}\text { ARR(Counted) } \\
\text { Test2 }\end{array}$ & $\begin{array}{l}\text { Difference } \\
\text { ARR \% Test1 }\end{array}$ & $\begin{array}{l}\text { Difference } \\
\text { ARR \% Test2 }\end{array}$ \\
\hline $\mathbf{1}$ & 19 & 18 & 41 & 38 & 5.6 & 7.9 \\
\hline $\mathbf{2}$ & 13 & 12 & 36 & 34 & 8.3 & 5.9 \\
\hline $\mathbf{3}$ & 15 & 15 & 48 & 46 & 0.0 & 4.3 \\
\hline $\mathbf{4}$ & 14 & 14 & 49 & 49 & 0.0 & 0.0 \\
\hline
\end{tabular}

Due to the human error in counting the breaths of the volunteer, the measured ARR has a difference of up to $8.3 \%$ with Test 1 of volunteer two, as seen in Table 7-3. With all the other tests a $\%$ difference were measured of less than $8 \%$, which were acceptable results. Therefore the system can detect the respiratory rate accurately.

\subsubsection{Temperature Results}

The temperature test results are summarized in Table 7-4. This indicates that the measured skin temperature values obtained were as expected for healthy subjects. An average temperature was calculated over the two minutes of testing for each test. 
TESTING AND RESULTS

Table 7-4 Temperature values from test data

\begin{tabular}{|c|c|c|}
\hline Volunteer & Average temperature $\left[{ }^{\circ} \mathrm{C}\right]$ - Test 1 & A verage temperature $\left[{ }^{0} \mathrm{C}\right]$ - Test 2 \\
\hline $\mathbf{1}$ & 36.8 & 36.9 \\
\hline $\mathbf{2}$ & 37.0 & 37.1 \\
\hline $\mathbf{3}$ & 37.0 & 37.1 \\
\hline $\mathbf{4}$ & 36.9 & 37.0 \\
\hline $\mathbf{5}$ & 37.1 & 37.2 \\
\hline
\end{tabular}

\subsubsection{Oxygen Saturation Results}

The testing of changes in $\mathrm{S}_{\mathrm{a}} \mathrm{O}_{2}$ is not included within the scope of this project and was hence not calculated for these tests. This is due to the complexity of the tests needed to measure low oxygen saturation values. The resting oxygen saturation values for each volunteer are summarised in Table 7-5.

Table 7-5 Oxygen saturation measured values

\begin{tabular}{|c|c|}
\hline Volunteer & $\mathrm{S}_{\mathrm{a}} \mathrm{O}_{2}[\%]-$ Test $\mathbf{1}$ \\
\hline $\mathbf{1}$ & 93.6 \\
\hline $\mathbf{2}$ & 94.3 \\
\hline $\mathbf{3}$ & 92.8 \\
\hline $\mathbf{5}$ & 93.1 \\
\hline
\end{tabular}

\subsection{Chapter Summary}

In this chapter the laboratory testing procedure was discussed and results obtained for five volunteers from the tests were presented. As can be seen from these results, the measurement of relevant vital signs was obtained and the two devices can be used together to monitor the vital signs of a psychiatric patient. 


\section{CHAPTER 8}

\section{CONCLUSIONS AND RECOMMENDATIONS}

The system functionality of the combination of the devices will be discussed in this chapter, and recommendations will be made with regards to future improvements that can be implemented.

\subsection{Conclusion}

In this thesis the primary objective was accomplished by designing and developing prototype devices that can monitor psychiatric patients' vital signs during sedation and seclusion. Each of the two devices contributes to the monitoring of the patient. An evaluation matrix was used to develop the mechanical concepts. These concepts were discussed in various meetings with experts which resulted in the decision to develop the two devices. The implementation of two devices resulted in a complete measurement of the most important vital signs of the patient, except blood pressure. All the design considerations were discussed, which included the development of electronic circuit diagrams and the relevant mechanical designs.

Prototypes of the two developed devices were implemented and the devices were tested on five healthy volunteers to verify the different sensors. The four main objectives of the project were persistently considered during the design of these devices, as shown in Chapter 1.2. Both prototypes were designed to be as small as possible considering the mechanical, physiological and electronic limitations for each device. The dorsal mounted device's placement on the body and the safety glove ensured that objective 2 was accomplished for both devices. The mechanical design implementations ensured that no wires are visible or accessible which also ensures that objective 2 is implemented in the mechanical designs. Objective 4 was accomplished by the development of the Matlab GUI that monitors and processes the vital sign data. The implementation of continuous blood pressure monitoring was added as a possible objective but after development of the continuous blood pressure monitor no usable results could be obtained.

The mechanical strength of the prototypes was not tested, since only a "proof-ofconcept" design was undertaken in this thesis. The prototypes could not be tested on psychotic patients in a psychiatric environment due to the limitation of the mechanical strength. Overall, however it can be concluded that the objectives of the thesis were adequately met in that a proof-of-concept was established. To 
commercialise this prototypes, further design and testing should be considered as discussed in Section 8.2.

\subsection{Recommendations}

The main unit (the dorsal mounted device) can be used without the safety glove to monitor all the variables excluding oxygen saturation. This can be implemented when the patient is totally uncontrollable or irritated by the safety glove. The dorsal mounted device cannot be accessed by the patient and is therefore designed as the main unit for monitoring.

The Bluetooth connection in the safety glove device can be substituted by a small low power wireless connection between the safety glove device and the dorsal mounted device. The PPG data can then be transmitted to the dorsal mounted device and the analogue input channels of the MCU can be used to sample the PPG signals and send this PPG data with the other sensors over one Bluetooth connection. This design will eliminate the use of the Bluetooth module on the safety glove device and will save battery power. This was not implemented as the dorsal mounted device was already manufactured and could not accommodate this change in design. Therefore the safety glove device wireless connection is implemented using a separate dedicated Bluetooth connection.

The mechanical design of the dorsal mounted device can be transformed using a larger but thinner surface area device and then moulding the device in the shape of the back of a person. This will ensure that the patient can lie down on his back and will not feel the device. This was not implemented due to the cost of developing a mould.

The implementation of a non-invasive continuous blood pressure monitor should be further investigated for use in the safety glove device. A possible pulse wave velocity (PWV) method shown by McCombie et al. [53] using pulse transit time (PTT) techniques can be further investigated.

Mechanical strength tests should be done on the prototypes to ensure that the prototypes can be used in a psychiatric patient environment. The prototype should be further developed and tests on psychotic patients in psychiatric hospital environment should be undertaken. The prototypes should also be tested and verified that it fulfils electromagnetic compatibility (EMC) regulations, before a commercial product can be implemented. 


\section{APPENDICES}

\section{Appendix A1 - Continuous Non-Invasive Blood Pressure Monitor}

There is a clinical need for long term continuous blood pressure monitoring. The use of traditional sphygmomanometer to measure systolic and diastolic brachial pressure should be substituted by a smaller cuffless design that can measure continuously without impeding blood flow. A patient's blood pressure can fluctuate in a very short time [54]. Therefore the continuous measurement will ensure that beat to beat measurement is done, which will assist in a clinical observation of the patient's cardiovascular functions [26]. Several types of instruments that are based on the vascular-unloading principle (Penaz method) have been commercially produced and designed to meet the requirements of beat to beat measurement of blood pressure [55]. These instruments are widely used in clinical settings. A small cuff attached around the index finger is used. This method impedes blood flow when measuring for long periods at a time. Therefore the Portapres device uses two small cuffs, one on the index finger and one on the middle finger. This ensures that the measurement can be switched periodically every 30 minutes between these two fingers to ensure the circulation of blood in the measured fingers. This is a logical idea, but the size of the system because of the additional equipment needed, makes it impractical for the user to use in an ambulatory setting [56]. A few known methods and other designs will be discussed regarding continuous blood pressure monitoring using non-invasive techniques.

\section{Portapres Commercial Blood pressure monitor.}

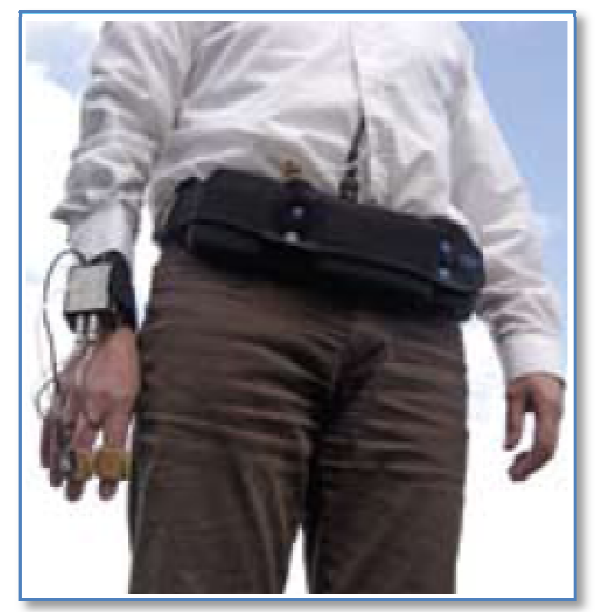

Figure A-1 Portapres portable continuous blood pressure monitor [57] 
The Portapres system shown in Figure A-1 uses the volume-clamp method which was first introduced by Peñáz in 1967 [55]. The beat to beat blood pressure is measured with this device. The Portrapres uses the Finapres ${ }^{\mathrm{TM}}$ technology which is based on an advanced non-invasive blood pressure measurement. The volume clamp method consists of a finger cuff, inflatable bladder and an infrared plethysmograph. An infrared diode and a photodiode are used to produce the photo plethysmograph signal. The same principle is used as in the pulse oximeter design. The blood absorbs the infrared light and an AC pulsation of the arterial blood diameter is detected using the photodiode. The following steps are used to determine the blood pressure [58]:

- Determine the proper unloaded diameter of the arteries in the fingers. This will be when the intra-arterial pressure and the finger cuff pressure are equal, which leads to the transmural pressure across the finger walls that will be zero.

- At this stage the arteries are kept at this unloaded diameter by clamping the arteries with the finger cuff using the fast cuff pressure control system.

- A set-point or target value is set by the servo-controller. The measure photo plethysmograph signal (unloaded diameter) is compared to the set-point.

- The "error signal" (the amplified difference between the set-point and the measured unloaded diameter) is used to control the fast pneumatic proportional valve in the main unit.

- This valve is then used to drive the air compressor so that the correct air pressure is supplied to the finger cuff [58].

Figure A-2 shows the volume clap methodology and mechanism used in the Portapres system.

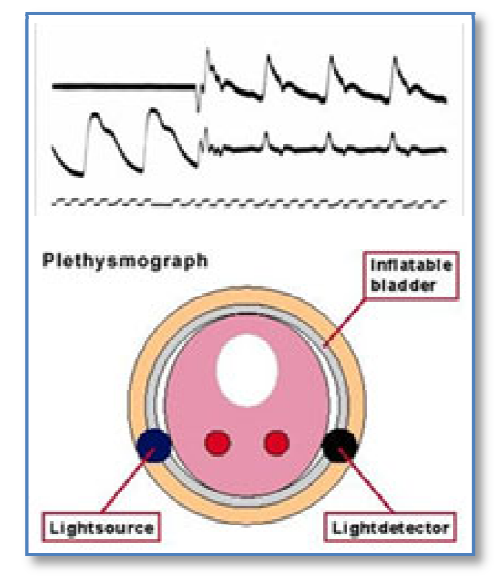

Figure A-2 Volume clap methodology and mechanism [58] 


\section{Pulse Wave Velocity (PWV) Method}

With the PWV method the pulse transit time (PTT) should be calculated. To measure the PTT two in-line photo plethysmograph (PPG) sensors are used. The one sensor is placed on the ulnar artery (wrist) with the other sensor on the digital artery (little finger) as shown in Figure A-3 [53]. The wrist sensor uses the reflectance oximetry method where the LED and photodiode are placed next to each other over the ulnar artery, and the sensor on the digital artery uses the transmission method where the LED and photodiode are placed on each side of the digital artery. Every heartbeat is used to calculate the time between the two pulses measured with the two devices. This is known as the pulse transit time (PTT). The onset of each of the two waves of the same heartbeat is used to calculate the PTT. The onset marker is defined as the point on the PPG wave where the wave has reached $5 \%$ of the peak systolic value [59].

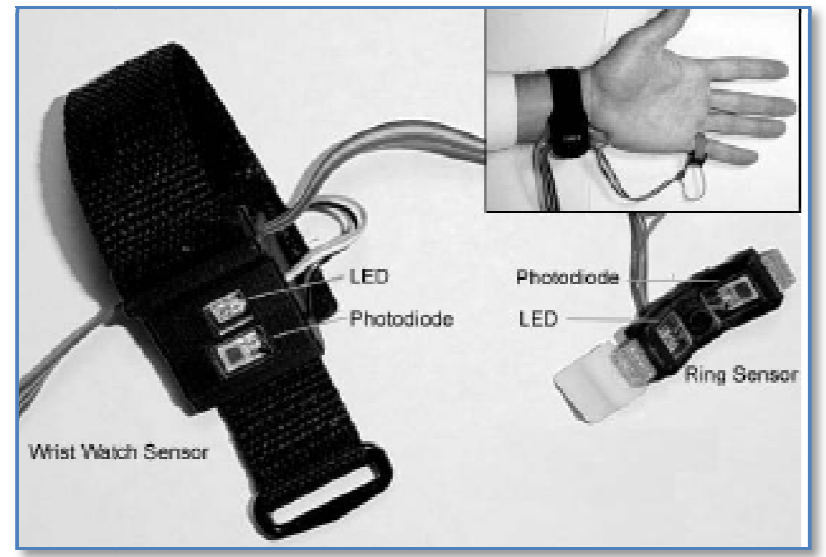

Figure A-3 Peripheral pulse wave velocity device [59]

The Finapres ${ }^{\mathrm{TM}}$ BP monitor discussed above was used to verify the PTT method. Both these devices were connected to one volunteer to show the correlation between the two methods used to calculate continuous blood pressure. The height of the hand was selected at two different heights to show the change in blood pressure due to the change in height. The hand was kept at the same hight then changed to the second position $(15 \mathrm{~cm}=11.0 \mathrm{mmHg})$. Figure A-4 shows the comparison between the Finapres ${ }^{\mathrm{TM}}$ blood pressure monitor measurement at these two heights and the PWV method at the same two heights on the same hand at the identical point in time [59]. 


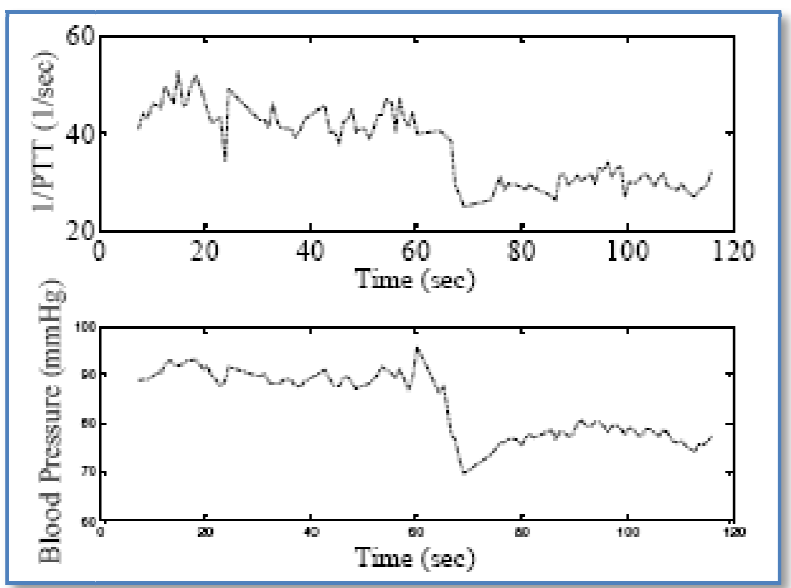

Figure A-4 Comparison of PWV data and BP data at two different heights [59]

\section{Accuracy Assessment of a Noninvasive Device for Monitoring Beat- by-Beat Blood Pressure in the Radial Artery Using the Volume- Compensation Method [56]}

For using the volume compensation method in this system the radial artery was chosen as a measuring site. The system is divided into three main components.

- A personal computer having a DSP-board installed

- An electromechanical unit (air pump, electro pneumatic converter (EPC), pressure sensor and associated components)

- An liquid crystal display (LCD) monitor

The whole system can be seen in Figure A-5. To avoid venous blockage during longterm monitoring a new incompletely occlusive pad-type cuff that is mounted inside a C-type rigid bracelet is used. Inside of the pad-type cuff a PPG based design is also used for detecting the vascular volume changes. This PPG sensor consists of two infrared LEDs $(940 \mathrm{~nm})$ and one photodiode (PD). The series connected LEDs are placed on each side of the radial artery to ensure that the measurement of a pulsatile signal is obtained. The PD is placed between the two LEDs over the artery as shown in Figure A-5 (left top corner). Simulink software toolbox from Matlab is used to control the servo control system. This is done using a proportional integral differential (PID) compensator circuit [56]. 


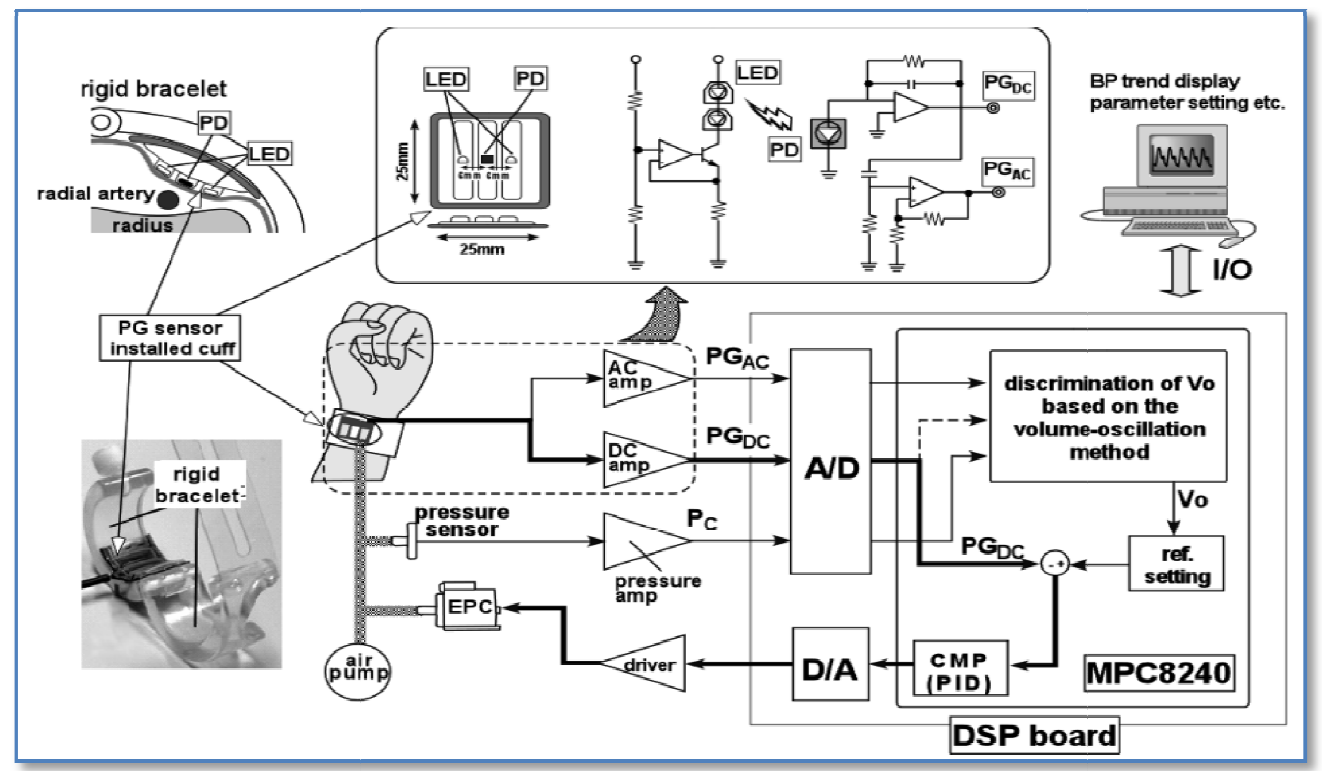

Figure A-5 Schematic block diagram of the prototype system [60]

\section{Estimate Blood Pressure Using a Hemodynamic Model of the Systemic Circulation together with a Kalman Filter [26]}

As discussed in Chapter 3.3, Yang et al. [26] developed a new method to estimate blood pressure using a developed hemodynamic model of the system circulation together with a Kalman filter design to estimate continuous blood pressure. The hemodynamic system is designed so that the observable/unobservable subspace decomposition technique should be used to calculate the blood pressure characteristics. The first step is to develop this hemodynamic model. This twodimensional mathematical model is derived from observing the small arterial blood flow segment in a digital artery. The entire arterial stream from the heart (proximal boundary) to the capillary (distal boundary) is calculated in a model. An extended Windkessel model [61] is used for the upstream and a classic Windkessel model [61] is used for the downstream to ensure that this entire arterial stream model is kept uncomplicated [26]. Figure A-6 shows the entire arterial stream model used. This includes the digital arterial segment with the upstream and downstream components. 


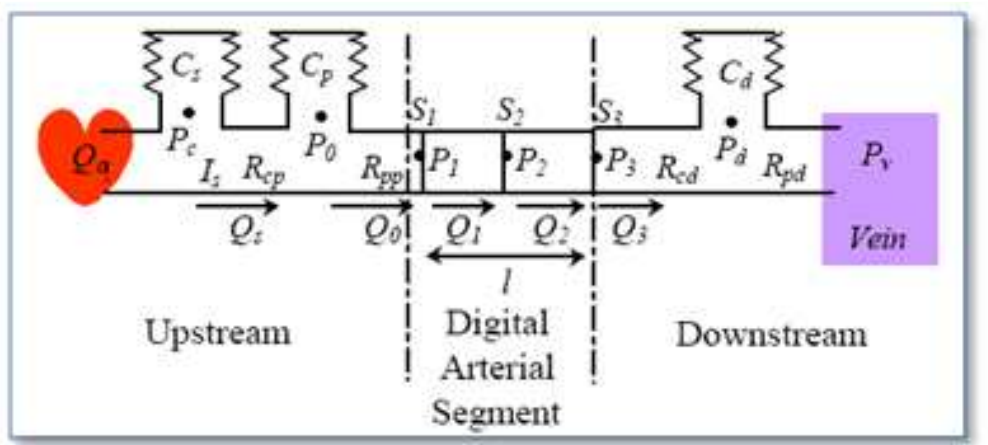

Figure A-6 Upstream, digital arterial segment and downstream model [26]

Now that we have the model design for the entire arterial stream a mathematical model should be calculated form Figure A-6.

For the upstream blood flow the following model is calculated:

$$
P_{1}=\frac{\sqrt{\pi} E e}{S_{1} \sqrt{S_{0}}}\left(S_{1}-\frac{Q_{1}-Q_{0}}{\Delta Z}-\sqrt{S_{0} S_{1}}\right)
$$

For the downstream blood flow the following model is calculated:

$$
\begin{aligned}
& Q_{N}=\frac{P_{N}-P_{d}}{R_{c d}} \\
& P_{N}=\frac{\sqrt{\pi} E e}{S_{N} \sqrt{S_{0}}}\left(S_{N}-\frac{\cap}{2 E} \frac{Q_{N}-Q_{N-1}}{\Delta Z}-\sqrt{S_{0} S_{N}}\right)
\end{aligned}
$$

Using the upstream and downstream the entire arterial model is the calculated as:

$$
\begin{aligned}
& x=\left[\begin{array}{lllllllll}
P_{c} & Q_{s} P_{1} & Q_{1} & \ldots & Q_{N-1} S_{1} & \ldots & S_{N} & P_{d}
\end{array}\right]^{T}:(2 N+3) \times 1 \\
& u=\left[Q_{c}, P_{V}\right]^{T}:(2 \times 1)
\end{aligned}
$$

with the state space representation of this extended dynamic model:

$$
\dot{x}=A x+B u
$$


APPENDICES

where

$$
\begin{aligned}
& A=\left[\begin{array}{c}
A_{\text {up }: 3 \times(2 N+3)} \\
A_{\text {local }(2 N-1) \times(2 N+3)} \\
A_{\text {down: } 1 \times(2 N+3)}
\end{array}\right]:(2 N+3) \times(2 N+3) \\
& B=\left[\begin{array}{cc}
\frac{1}{C_{s}} & 0 \\
0 & 0 \\
\vdots & \vdots \\
0 & 0 \\
0 & \frac{1}{C_{d} R_{p d}}
\end{array}\right]:(2 N+3) \times 2
\end{aligned}
$$

A regularly assumed pattern is used as the input to the system for the cardiac output. When evaluating the overall system is it found to be unobservable as expected. But when observability analysis is done the estimation of blood pressure for the small digital artery segment can be made from the observable subspace. After calculating this, a low order Kalman filter is designed for this specific subspace [26].

A wearable prototype was designed as shown in Figure A-7. The Kalman filter was designed for this specific prototype which includes two PPG sensors and one EIP sensor as shown in Figure A-7.

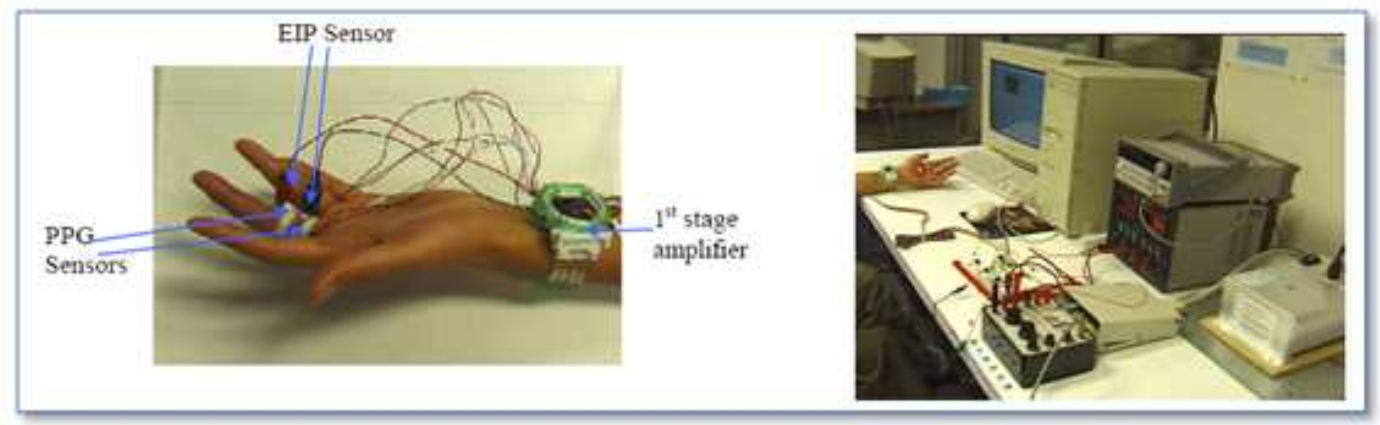

Figure A-7 Prototype design of EIP and PPG sensors [26] 
The PPG sensors (LED and photodiode) are used to monitor the variations of the arterial wall diameter at a specific location. The two sensors' observation functions are defined as:

$$
y_{1}(t)=S_{1}(t), y_{2}(t)=S_{N}(t)
$$

An EIP system is used to measure the volumetric change in a segment where an EIP measures the change in electrical impedance over an arterial segment defined by the area surrounded by the measuring electrodes. The output of the EIP system is defined with $\mathrm{y} 3$ as a state variable below in equation A.1.9:

$$
y_{3}(t)=V(t)=\frac{1}{2} S_{1} \Delta z+\left(S_{2+\cdots+} S_{N-1}\right) \Delta z+\frac{1}{2} S_{N} \Delta z
$$

Defining $y(t)=[y 1(t), y 2(t), y 3(t)]^{T}$, the observation equation can finally be defined as

$$
y(t)=C x(t)
$$

where

$$
C=\left[\begin{array}{ccccccccc}
0 & \cdots & 0 & 1 & 0 & \cdots & 0 & 0 & 0 \\
0 & \cdots & 0 & 0 & 0 & \cdots & 0 & 1 & 0 \\
0 & \cdots & 0 & \frac{\Delta z}{2} & \Delta z & \cdots & \Delta z & \frac{\Delta z}{2} & 0
\end{array}\right]: 3 \times(2 N+3)
$$

Summarizing the above formulations the arterial hemodynamic monitoring system can be represented as:

$$
\begin{aligned}
& \dot{x}=A x+B u \\
& y=C x \\
& p=g^{T} x
\end{aligned}
$$

\section{- Designing the EIP recording system}

Now that the hemodynamic mathematical model is defined, the sensor design should be explained. The design and implementation of the PPG sensors are easily compared to that of the EIP recording system. Figure A-8 shows the block diagram of this system with the steps to calculate the volumetric change in the artery. 
APPENDICES

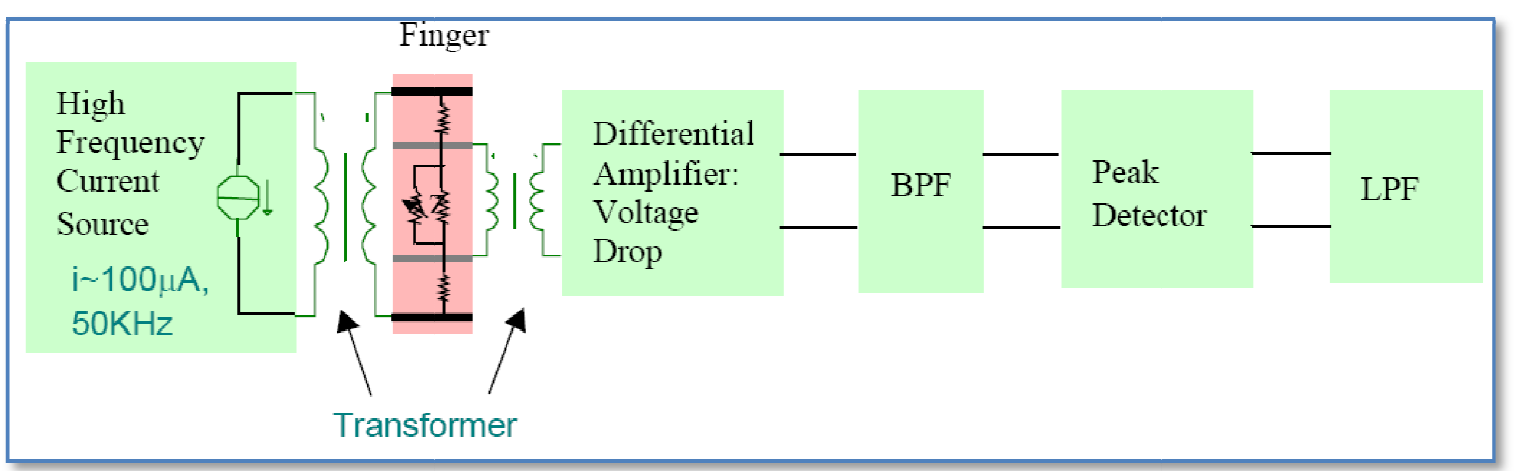

Figure A-8 Block diagram of EIP measuring system [26]

The EIP consists of five main blocks (excitation module, voltage preamplifier, bandpass filter, peak detector and lowpass filter).

\section{Continuous Blood Pressure Measurement Conclusion}

After evaluating all the designs discussed above it was found that none of these blood pressure measurement systems could be used, in a psychotic patient environment due to either the size of the measurement equipment or the inaccurate data obtained from the devices. Some of the systems need a reference calibration input which is not always possible. Therefore further research into the continuous blood pressure monitoring for psychotic patients should be undertaken. 


\section{Appendix A2 - Commercial and Patented Monitoring Devices}

Table A-1 shows a few commercial and patented monitoring devices for ECG, oxygen saturation and blood pressure. The devices shown in Table A-1 cannot be used in a psychotic patient environment. They can measure a single needed vital sign but a psychotic patient monitoring device should be small and placed at a specific location on the body where the device will not irritate the patient.

Table A-1 Commercial and patent monitoring devices

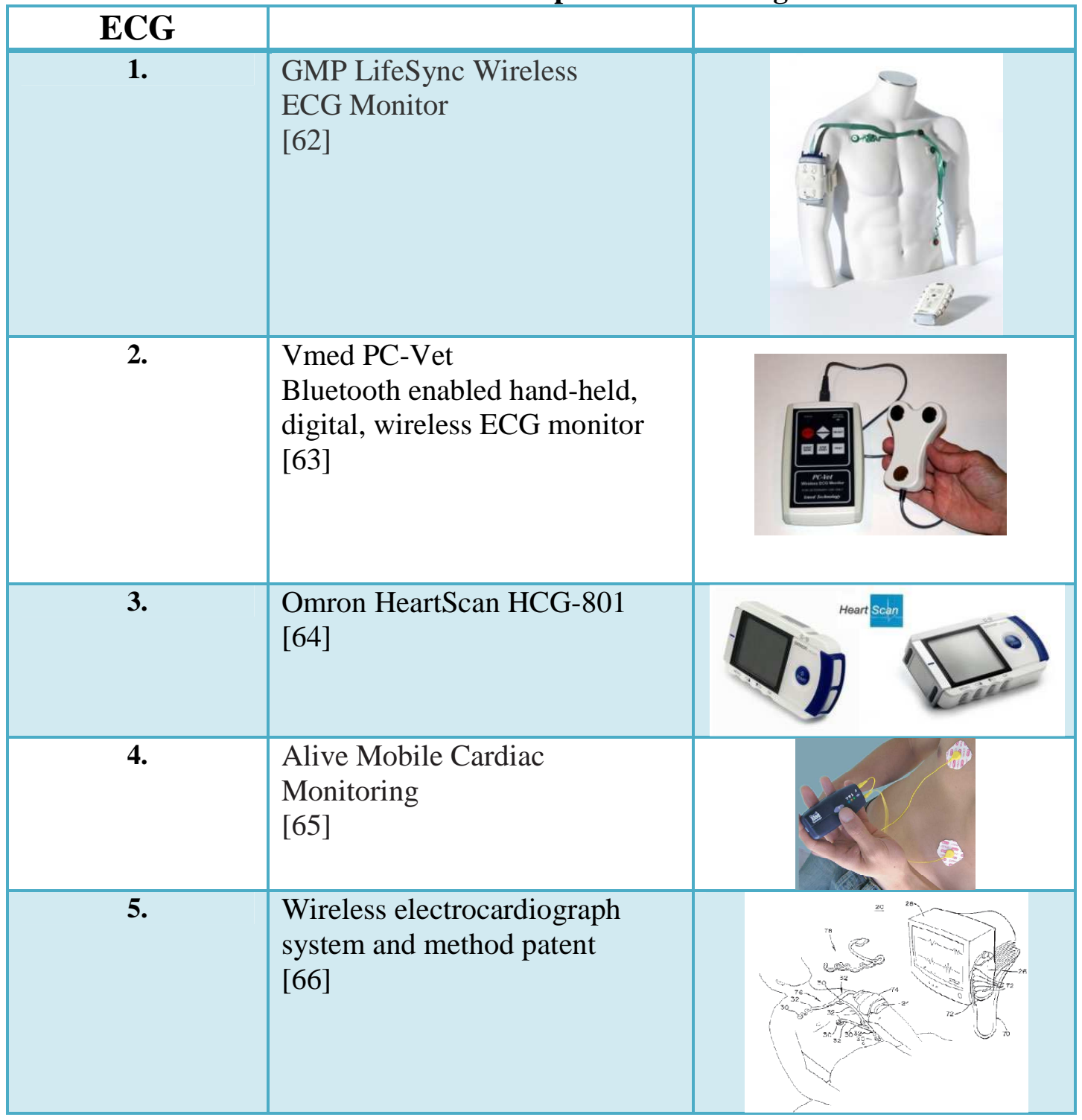




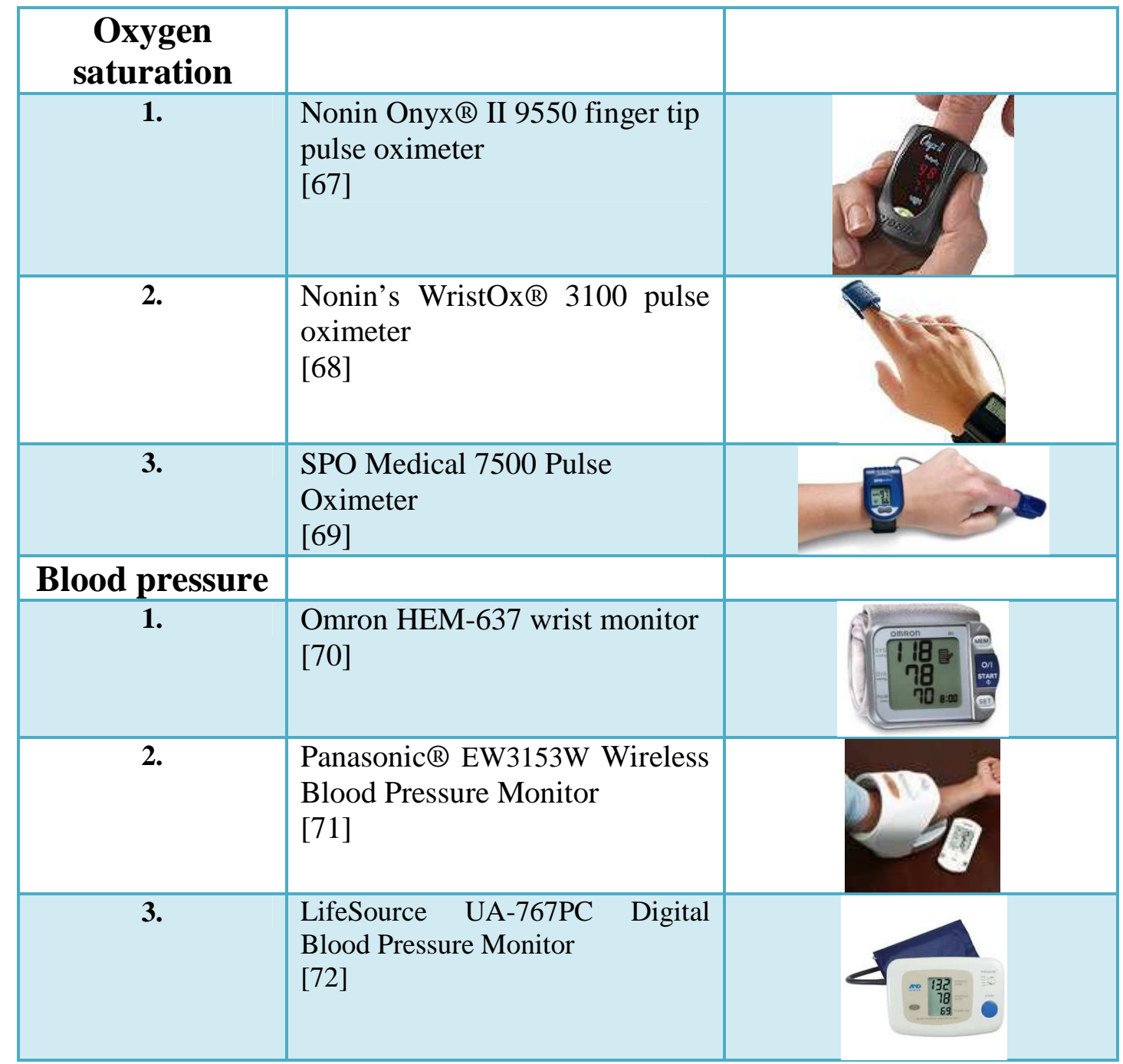




\section{Appendix A3 - Wireless Commercial Pulse Oximeter Products}

After development of the Bluetooth enabled wireless pulse oximeter inside the safety glove, a further research study revealed some relevant commercial products shown in Figure A-9 and Figure A-10. These also measure oxygen saturation and transmit it wirelessly using Bluetooth protocol to a central monitoring station. It is very simular to that of the safety glove device, except that the safety glove device has an accelerometer which monitors the patient's movement. When detected by the microcontroller that the patient moves for more than a predefined value, the sending of PPG data will be terminated until the movement of the patient terminates. This feature is used to ensure that no false readings are obtained for oxygen saturation during the monitoring of an aggressive psychotic patient.

\section{Alive Wireless Pulse Oximeter}

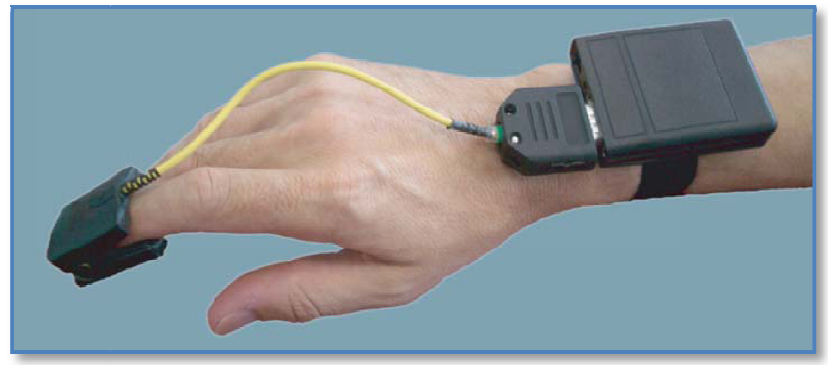

Figure A-9 Alive wireless Bluetooth enabled pulse oximeter [73]

\section{Nonin 4100 Bluetooth Pulse Oximeter}

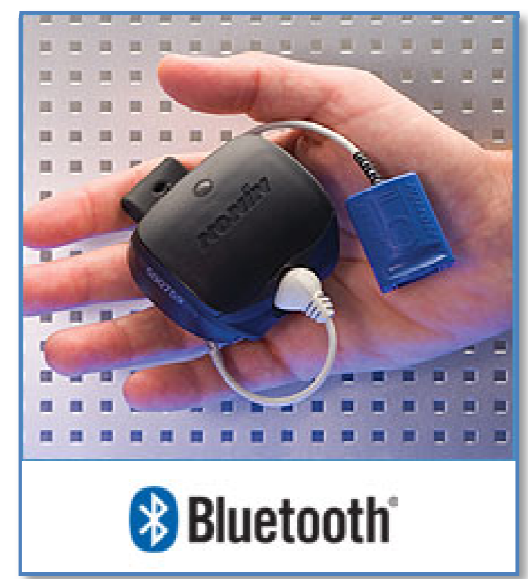

Figure A-10 Nonin 4100 Bluetooth enabled pulse oximeter [74] 
APPENDICES

Appendix B1 - PCB of Dorsal Mounted Device

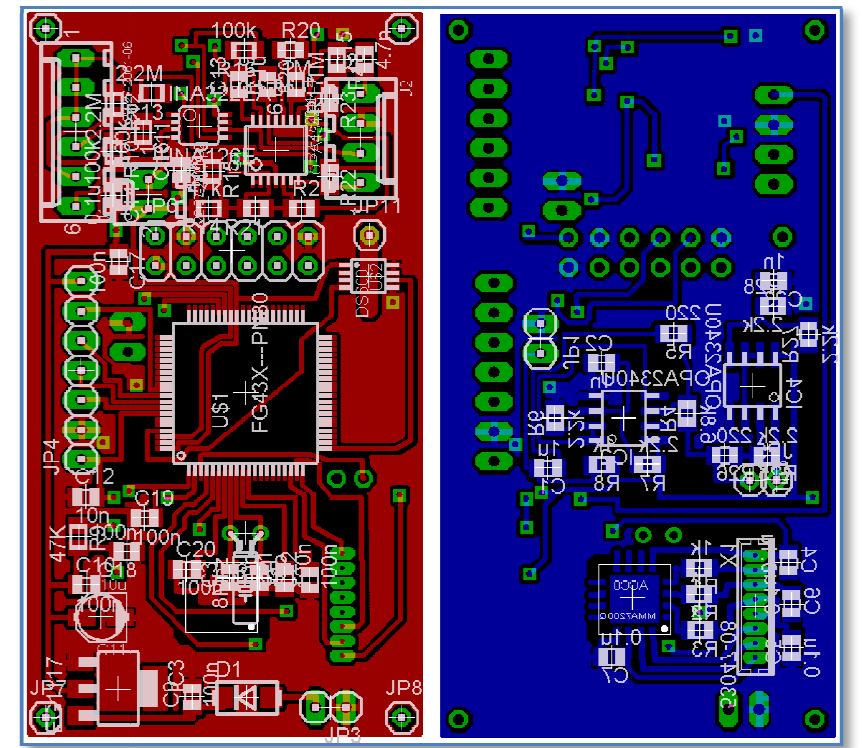

Figure A-11 Top (left) and bottom (right) of PCB

Appendix B2 - PCB of Safety Glove Device

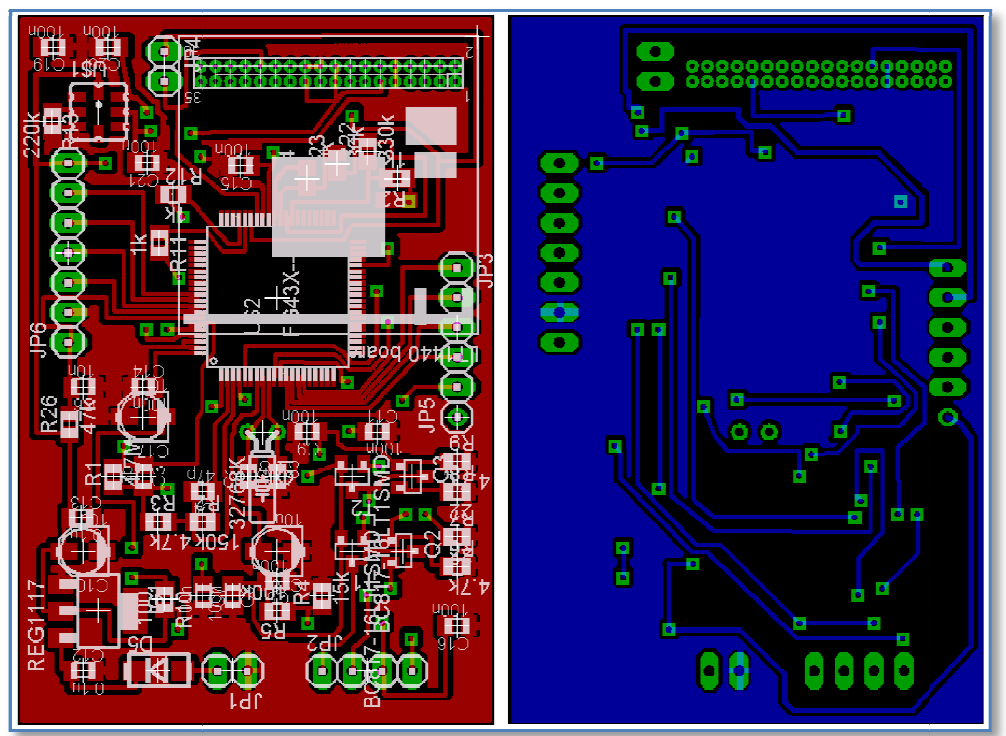

Figure A-12 Top (left) and bottom (right) of PCB 


\section{Appendix C1 -CAD Design of Dorsal Mounted Device}

I. Enclosure

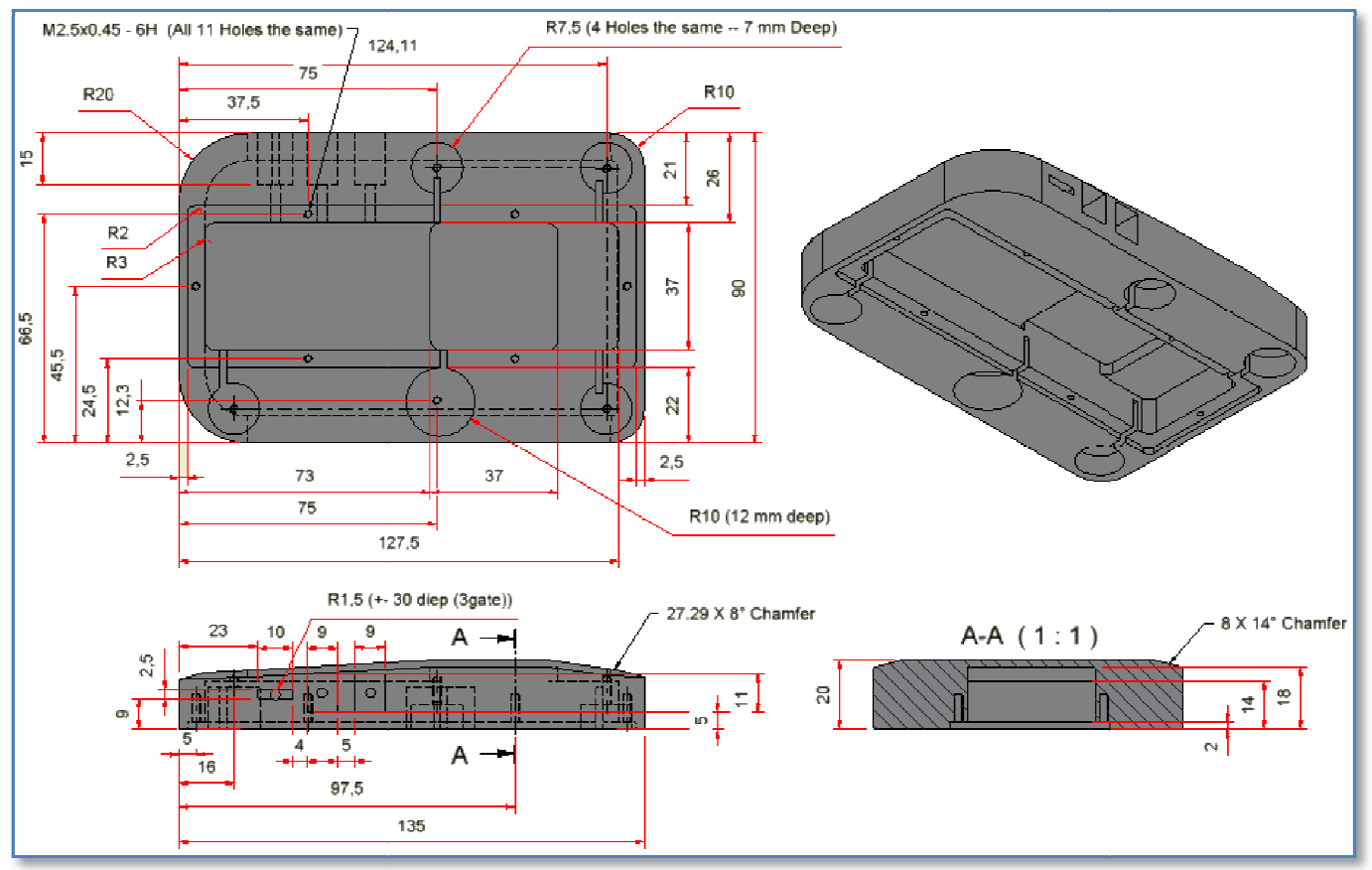

Figure A-13 Enclosure manufacturing drawing

II. Baseplate

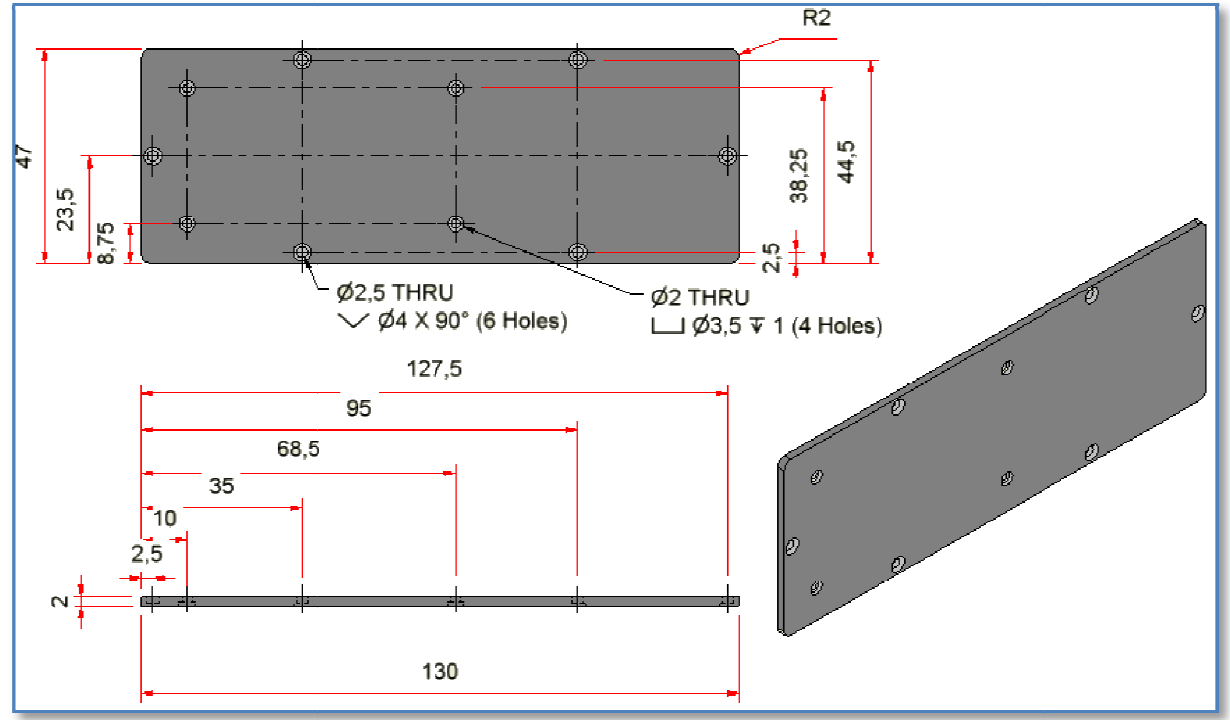

Figure A-14 Baseplate manufacturing drawing 
APPENDICES

III. Sound Unit

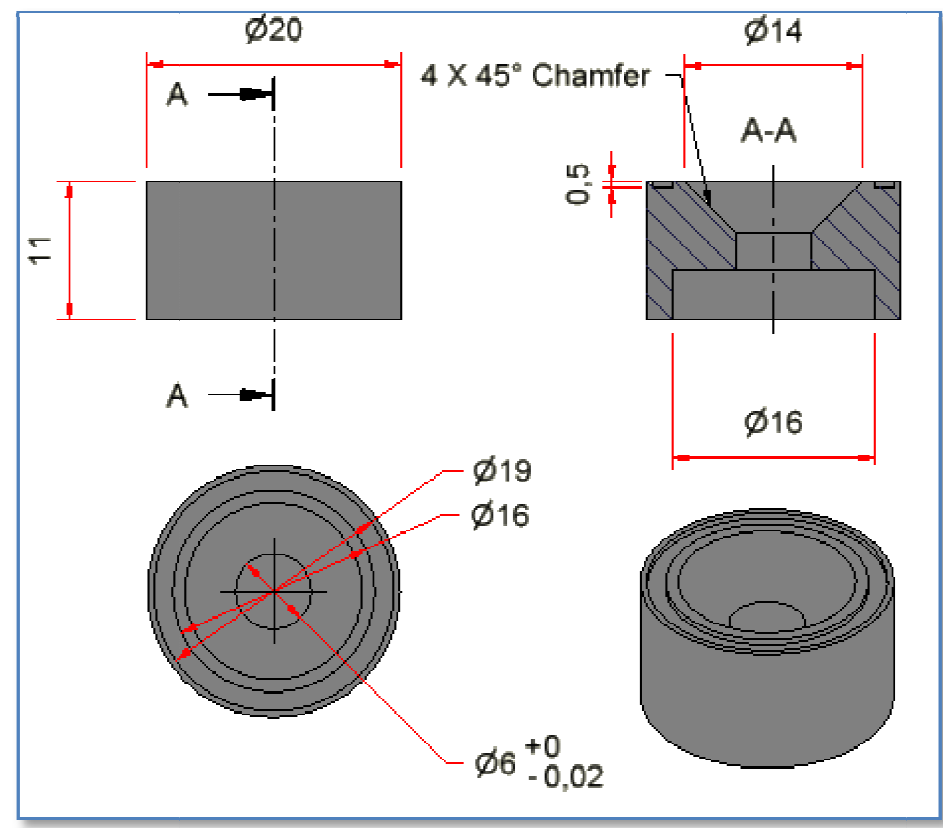

Figure A-15 Sound unit manufacturing drawing

Appendix C2 - CAD Design of Safety Glove Enclosure

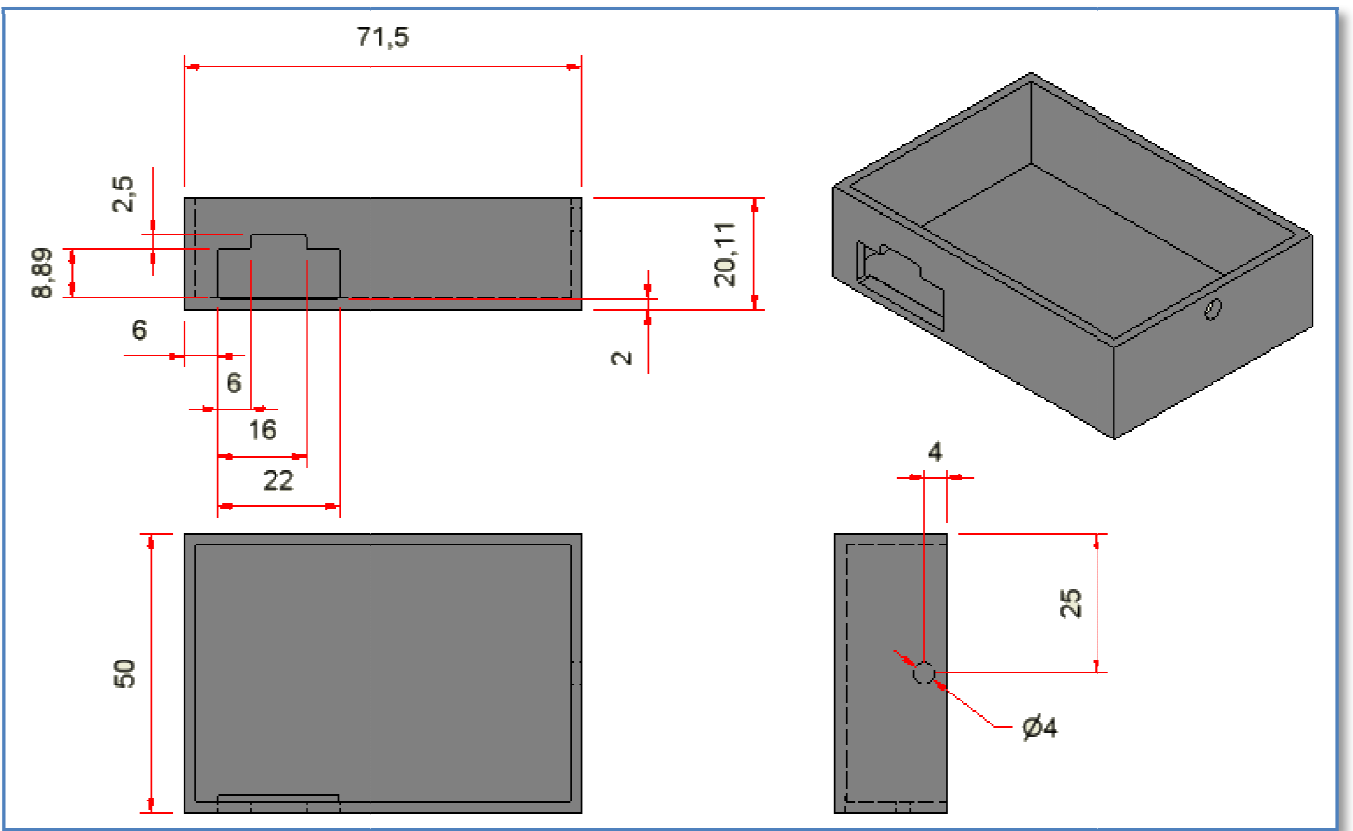

Figure A-16 Electronic protection enclosure manufacturing drawing 


\section{Appendix D1 - Project Planning}

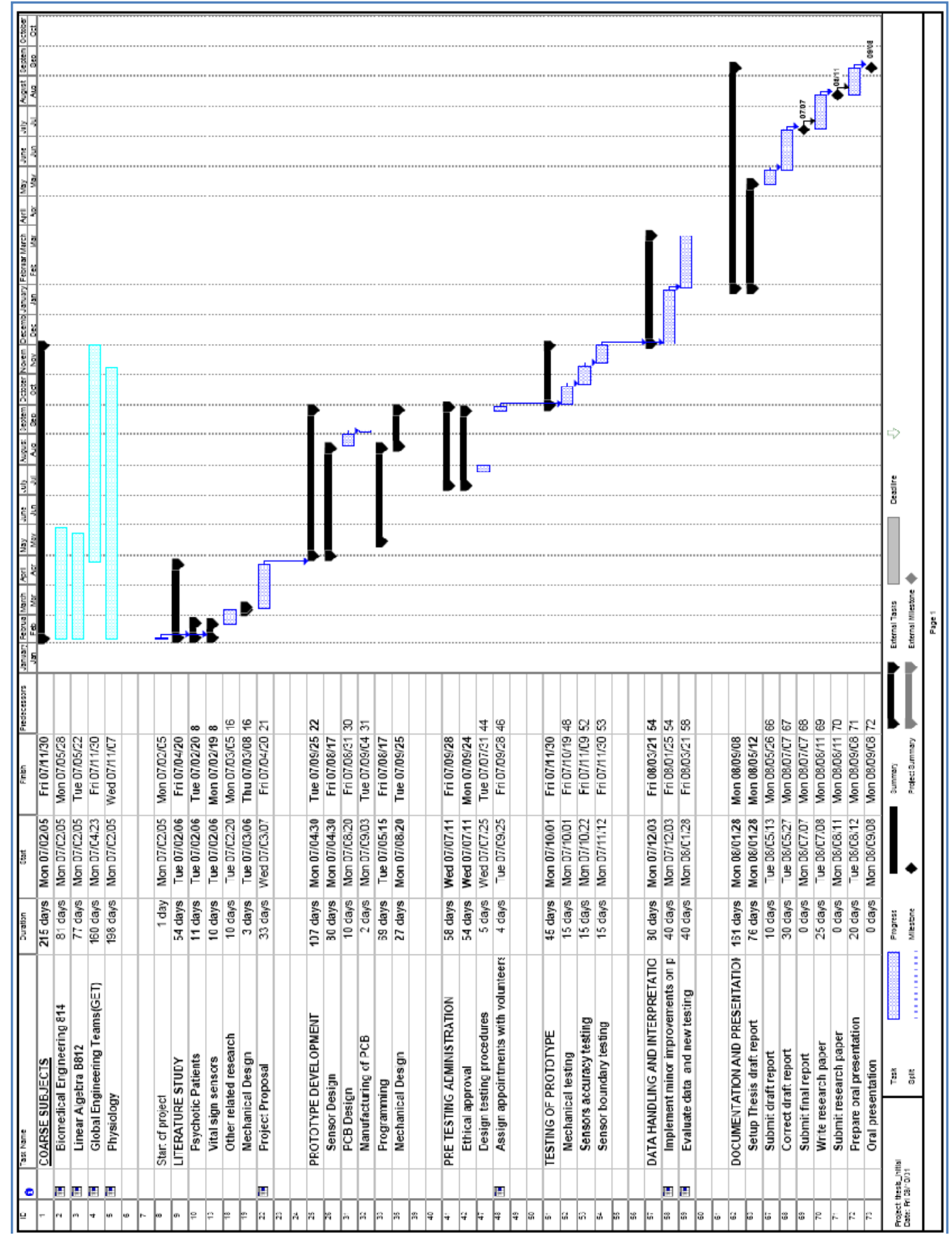

Figure A-17 Gantt chart 
APPENDICES

\section{Appendix D2 - Project Prototypes Expenses}

The following table includes the costs for each component for the development of the two prototypes.

Table A-2 Estimated prototype expenses

\begin{tabular}{|l|cl|}
\hline $\begin{array}{l}\text { Component } \\
\text { Bluetooth } \\
\text { modules }\end{array}$ & Number & \multicolumn{1}{c|}{ Total Cost } \\
\hline PCB & 2 & \\
\hline $\begin{array}{l}\text { Saturation } \\
\text { Finger Probe }\end{array}$ & 2 & R 500 \\
\hline Batteries & 2 & R 1000 \\
\hline Enclosures & 2 & R 200 \\
\hline Electronics & -- & R 400 \\
\hline Total & & R 4000 \\
\hline
\end{tabular}




\section{REFERENCES}

[1] Weiss, EM. 2002. How the Courant Conducted its Investigation. [Online] Available at:

http://www.charlydmiller.com/LIB05/1998hartfordinvestigation.html. [2007, February 13].

[2] NMHA. 2006. National Mental Health Association. [Online] Available at: http://www1.nmha.org/position/ps41.cfm. [2007, January 16].

[3] South African government regulation. 2003. Mental health care act regulations. [Online] Available at:

http://www.info.gov.za/regulations/2003/24384/index.html. [2007, January 16].

[4] Waller, A.D. 1889. One of the electromotive changes connected with the beat of the mammalian heart, and the human heart in particular. Phil Trans B 180:169.

[5] Akay, M. 2006. Wiley Encyclopedia of Biomedical Engineering. Vol 2. John Wiley \& Sons, Inc, New Jersey.

[6] Biolog. 2007. Electrode Placement. [Online] Available at: http://www.biolog3000.com/electrode.htm. [2007, March 16].

[7] College of Science and Mathematics - Heart. [Online] 2007. Available at: http://csm.jmu.edu/biology/danie2jc/heart.htm. [2007, November 22].

[8] Enderle, J., Blanchard, S. \& Bronzino, J. 2005. Introduction to Biomedical Engineering. $2^{\text {nd }}$ edn. Elsevier Academic Press, San Diego.

[9] Martini, F.H \& Bartholomew, E.F. 2003. Essentials of Anatomy \& Physiology. $3^{\text {rd }}$ edn. Pearson Education Inc, New Yersey.

[10] Brodnick, D. 2005. Methods and Systems for Monitoring Respiration. United States of America.

[11] Mather, B. \& Winter, D. 1992. Respiration Rate Monitor. United States of America. 


\section{REFERENCES}

[12] UFI.: 1132 Pneumotrace II(R) by UFI. [Online] Available at: http://www.ufiservingscience.com/DS11321.html. [2008, February 25].

[13] ABE 2062 Biology for Engineers I. Lecture 20. 2006. [Online] Available at: http://www.agen.ufl.edu/ chyn/age2062/lect/lect_20/30_12.GIF. [2008, August 08].

[14] Pandian, P.S., Mohanavelu, K., Safeer, K.P., Kotresh, T.M., Shakunthala, D.T., Gopal, P. \& Padaki, V.C. 2007. Medical Smart Vest - Wearable MultiParameter Remote Physiological Monitoring System. Vol. 30. Engineering and Physics.

[15] Simple Diagnosis and Therapy Monitoring System for Sleep-Related Respiratory Disorders - Somnocheck. [Online] Available at: http://www.weinmann.de/en/home_homecare/sleep_diagnosis/somnocheck_eff ort/. [2008, January 20].

[16] NASA: LifeGuard - Wireless Physiological Monitor. [Online] Available at: http://www.nasa.gov/centers/ames/research/technology-onepagers/lifeguard.html. [2008, August 08].

[17] Richard, N., Lopez, S., Khair, M., Ghaem, S. \& Olson, W. 2007. Programmable Wireless Electrode System for Medical Monitoring. Patent: 7171166 United States of America.

[18] Money, E., Caldwell, R. \& Sciarra, M. 1999. Vital Sign Remote Monitoring Device. Patent: 5919141 United States of America.

[19] Besson, M., von Czettriz, G. \& Bax, R. 2007. Wireless Medical Diagnosis and Monitoring Equipment. Patent: 7215991 United Stated of America.

[20] Gallant, S.L. \& Markle, W.H. 2004. Method and Apparatus for Monitoring Physiologic Parameters of a Living Subject. Patent: 6705990 United States of America.

[21] Banet, M.J. \& Visser, H. 2007. Device, System and Method for Monitoring Vital Signs. Patent: 7238159 United States of America.

[22] Paradiso, R. Wearable Health Care System for Vital Signs Monitoring. Vol. Proc of the 4th Annual IEEE Conference on Information Technology Applications in Biomedicine. Prato,Italy. 


\section{REFERENCES}

[23] Jovanov, E., Milenkovic, A., Otto, C., De Groen, P., Johnson, B., Warren, S. \& Taibi G. 2005. A WBAN System for Ambulatory Monitoring of Physical Activity and Health Status: Applications and Challenges. Engineering in Medicine and Biology Society, IEEE-EMBS. 27th Annual International Conference, Vol. 3810-3813. ISBN: 0-7803-8741-4.

[24] Anliker, U., Ward, J.A., Lukowicz, P.,Tr“oster, G., Dolveck, F., Baer, M., Keita, F., Schenker, E., Catarsi, F., Coluccini, L., Belardinelli, A. \& Shklarski, D. 2004. AMON: A Wearable Multiparameter Medical Monitoring and Alert System.. Information Technology in Biomedicine, IEEE Transactions, Vol. 415427. ISSN: 1089-7771. Switzerland.

[25] Ullman, D.G. 2002. The Mechanical Design Process. $3^{\text {rd }}$ edn. McGraw-Hill Professional.

[26] Zhang, Y., Yang, B. \& Asada, H.1999. Cuff-Less Continuous Monitoring Of Blood Pressure Using a Hemodynamic Model. MIT Home Automation and Healthcare Consortium: Proceedings of the first joint, 1999. Proc. Of 1999 first Joint BMES/EMBS Conference. Progress Report No.1-3.

[27] Barber, D.C., Lawford, P.V., Hose, D.R., Smallwood, R.H. \& Brown, B.H. 1998. Medical Physics and Biomedical Engineering. CRC Press. ISBN $0750303689,9780750303682$.

[28] Sena User Guide. Sena Technologies, Inc. 2008. [Online] Available at: http://www.sena.com/download/manual/manual_parani_esd-v1.1.4.pdf. [2008, March 15].

[29] ESD100, Sena Parani Datasheet. Sena Technologies, Inc. 2008. [Online] Available at: http://www.sena.com/download/datasheet/ds_parani_esd.pdf. [2008, March 15].

[30] Gerasimov, V. 2003. Every Sign of Life. Massachusetts Institute of Technology, PhD Thesis. [2008, January 18].

[31] Fulford-Jones, T.R.F., Wei, G. \& Welsh, M. 2004. A Portable, Low-Power, Wireless Two-Lead EKG System. Engineering in Medicine and Biology Society, Vol 1, pp. $2141-2144$.

[32] Horowitz, P. \& Hill, W. 1989. The Art of Electronics. $2^{\text {nd }}$ edn. Press Syndicate of the University of Cambridge. 


\section{REFERENCES}

[33] DS600 Maxim Datasheet. Maxim. 2007. [Online] Available at: http://datasheets.maxim-ic.com/en/ds/DS600.pdf.

[34] MMA7260QT Freescale Semiconductor: 3-Axis Acceleration Sensor.20042008. [Online] Available at:

http://www.freescale.com/webapp/sps/site/prod_summary.jsp?code=MMA7260 QT\&fsrch $=1$.

[35] MSP430x4xx Family Users Guide. Texas Instruments. 2007. [Online] Available at: http://focus.ti.com/lit/ug/slau056g/slau056g.pdf. [2007, October 10].

[36] MSPGCC. 2004. Msp430 Uart Baudrate Calculator. [Online] Available at: http://mspgcc.sourceforge.net/baudrate.html. [2008, January 15].

[37] RMSS. 1994. Ergonomic Design: Anthropometry. RSA-MIL-STD-127.

[38] Catalano, J.T. 2001. Guide to ECG Analysis. Lippincott Williams \& Wilkins. ISBN $0781729300,9780781729307$.

[39] Kraman, S.S., Wodicka, G.R., Pressler, G.A. \& Pasterkamp, H. 2006. Comparison of Lung Sound Transducers Using a Bioacoustic Transducer. Journal of Applied Physiology, Vol. 101. Manitoba, Canada.

[40] TLV1117-33 Texas Instruments. 1995-2008. [Online] Available at: http://focus.ti.com/docs/prod/folders/print/tlv1117-33.html.

[41] Cadsoft. Eagle . 2008. [Online] Available at: http://www.cadsoft.de/freeware.htm.

[42] Texas Instruments. 1995-2008. Medical Electronic Solutions. [Online] Available at:

http://focus.ti.com/apps/docs/appcategory.tsp?appId=270\&DCMP=TIHomeTra cking\&HQS=Other+OT+home_a_medical.

[43] Application Notes Abstract. Texas Instruments. 1995-2008. A Single-Chip Pulsoximeter Design Using the MSP430. [Online] Available at: http://focus.ti.com/general/docs/techdocsabstract.tsp?abstractName=slaa274. [2007, October 16].

[44] SLAA274. Texas Instruments. 2005. A Single-Chip Pulsoximeter Design Using the MSP430. [Online] Available at:

http://focus.ti.com/lit/an/slaa274/slaa274.pdf. [2007 October 16]. 
[45] HP. Hewlett-Packard. 1997. A New Family of Sensors for Pulse Oximetry. [Online] Available at: http://www.hpl.hp.com/hpjournal/97feb/feb97a7.pdf. [2008, June 15].

[46] HP. Hewlett-Packard. 1997. [Online] Available at: http://www.hpl.hp.com/hpjournal/97feb/feb97a7a.pdf. [2008, June 15].

[47] ADXL311Analog Devices. 1995-2008. Ultracompact $\pm 2 g$ Dual-Axis Accelerometer. [Online] Available at: http://www.analog.com/en/mems-andsensors/imems-accelerometers/adx1311/products/product.html.

[48] Texas Instruments. Design Considerations. 2007. ECG Electrocardiogram. [Online] Available at: http://focus.ti.com/docs/solution/folders/print/464.html. [2007, February 15].

[49] Levine, J., Munden, J. \& Thompson, G. 2007. ECG Interpretation Made Incredibly Easy!. $4^{\text {th }}$ edn. Lippincott, Williams \& Wilkins. ISBN 1582555648 , 9781582555645.

[50] Anuradha, B., Kumar, K.S. \& Reddy, C.V.V. 2008. Classification of Cardiac Signals Using Time Domain Methods. $3^{\text {rd }}$ edn. Asian Research Publishing Network (ARPN), Tirupati, India.

[51] Bio-Medical Instruments.2008. [Online] Available at: http://biomedical.com/news_display.cfm?newsid=63. [2008, August 01].

[52] Polar. Polar T61 Heart Rate Monitor. 1990-2008. [Online] Available at: http://heartratemonitors.com/t-61_transmitter.htm.

[53] McCombie, D., Reisner, A. \& Asada, H. 2006. Adaptive Blood Pressure Estimation from Wearable PPG Sensors Using Peripheral Artery Pulse Wave Velocity Measurements and Multi-Channel Blind Identification of Local Arterial Dynamics. Proc. of the 28th IEEE International Engineering in Medicine and Biology Conference. EMBC. New York, NY.

[54] Yamakoshi, K. \& Tanaka, S. 1996. Ambulatory Instrument for Monitoring Indirect Beat To-Beat Blood Pressure in Superficial Temporal Artery Using Volume-Compensation Method. Medical \& biological engineering \& computing, Vol. 34, pp. 441-447. 


\section{REFERENCES}

[55] Penaz, J. 1973. Photoelectric measurement of blood pressure, volume and flow in the finger. Dig. 10th International. Conference. Medical Biological Engineering. p104.

[56] Tanaka, S., Nogawa, M., Yamakoshi, T. \& Yamakoshi, K. 2007. Accuracy Assessment of a Non-invasive Device for Monitoring Beat-by-Beat Blood Pressure in the Radial Artery Using the Volume-Compensation Method. IEEE Transactions on Biomedical Engineering, Vol.54.

[57] FMS.: PORTAPRES. Finapres Medical Systems. 2007. [Online] Available at: http://www.finapres.com/customers/portapres.php. [2008, January 15].

[58] FMS.: VOLUME-CLAMP. Finapres Medical Systems. 2007. [Online] Available at: http://www.finapres.com/customers/volume_clamp.php. [2008, January 18].

[59] McCombie, D.B., Shaltis, P.A., Reisner, A.T. \& Asada, H.H. 2007. Adaptive Hydrostatic Blood Pressure Calibration: Development of a Wearable, Autonomous Pulse Wave Velocity Blood Pressure Monitor. Proceedings of the 29th Annual International Conference of the IEEE, Vol. 5. EMBS. Lyon.

[60] Tanaka, S., Gao, S., Nogawa, M. \& Yamakoshi, K. 2005. Non-Invasive Measurement of Instantaneous Radial Artery Blood Pressure-an Instrument Based On the Volume-Compensation Method. IEEE Eng. Med. Biol.,Vol. 24, pp. 32-37.

[61] Li, John K-J. 2000. The Arterial Circulation Physical Principles and Clinical Applications. Published by Humana Press, New York. ISBN 0896036332, 9780896036338.

[62] GMP Wireless Medicine Inc.: GMP LifeSync Wireless ECG Monitor. [Online] Available at: http://www.idsa.org/idea/idea2005/g179.htm

[63] vmedtech.: PC-VET Wireless ECG Monitor. [Online] 2007. Available at: $\mathrm{http}: / / \mathrm{www} . v m e d t e c h . c o m / w i r e l e s s \_e c g . h t m$

[64] Omron Europe. 2008. HeartScan Product Information. [Online] Available at: http://www.omron-healthcare.com/sitepreview.php?SiteID=184. [2008, August 04].

[65] Alive. 2008. Mobile Cardiac Monitoring. [Online] Available at: http://www.alivetec.com/pdf/heartmonitor_handout.pdf [2008, August 10]. 


\section{REFERENCES}

[66] Hopman, N.C., Williams, D.L. \& Lodato, F. 2003. Wireless Electrocardiograph System and Method. Patent: 6611705, United States of America.

[67] Nonin. 2008. Onyx Ii 9550 Finger Tip Pulse Oximeter. [Online] Available at: http://www.nonin.com/products.asp?ID=1\&sec=1\&sub=1. [2008, August 04].

[68] Nonin. 2008. Wrist Ox 3100 Pulse Oximeter. [Online] Available at: http://www.nonin.com/products.asp?ID=19\&sec=1\&sub=6. [2008, August 04].

[69] Turner Medical. 2008. SPO Medical 7500 Pulse Oximeter. [Online] Available at: http://www.turnermedical.com/SPO_PulseOx_7500_Pulse_Oximeter.htm. [2008, August 04].

[70] Omron. 2008. R6 (HEM-6000-E). [Online] Available at: http://www.omronhealthcare.com/sitepreview.php?SiteID=234. [2008, August 04].

[71] Panasonic. 2007. EW3153W Blood Pressure Monitor. [Online] Available at: http://www2.panasonic.com/consumer-electronics/support/PersonalHealthcare/Blood-Pressure-Monitors/model.EW3153W. [2008, August 04].

[72] Quickmedical. 2008. LifeSource UA-767PC Digital Blood Pressure Monitor. [Online] Available at:

http://www.quickmedical.com/LifeSource/UA767pc.html. [2008, August 04].

[73] Alive. 2008. Wireless Pulse Oximeter. [Online] Available at: http://www.alivetec.com/pdf/pulseox_handout.pdf. [2008, August 04].

[74] Nonin. 2008. 4100 Bluetooth ${ }^{\circledR}$ Oximeter. [Online] Available at: http://www.nonin.com/products.asp?ID=29\&sec $=2 \&$ sub=9. [2008, August 04]. 